\title{
Molecular and Functional Characterization of \\ Programmed Cell Death (PCD) in Toxoplasma gondii
}

\author{
Dissertation \\ for the award of the degree \\ "Doctor rerum naturalium" (Dr.rer.nat.) \\ of the Georg-August-Universität Göttingen \\ within the doctoral program Biology \\ of the Georg-August University School of Science (GAUSS) \\ submitted by \\ Ni Nyoman Ayu Dewi \\ from Denpasar, Indonesia (born on $10^{\text {th }}$ November 1977)
}

Göttingen, 2013 


\section{Thesis Committee}

1. Prof. Dr. Uwe Groß

(Institute for Medical Microbiology, Department of Medical Microbiology)

2. Prof. Dr. Ernst A. Wimmer

(Johann-Friedrich-Blumenbach-Institute of Zoology and Anthropology, Department of Developmental Biology)

\section{$\underline{\text { Supervisor }}$}

Prof. Dr. Carsten G.K. Lüder

(Institute for Medical Microbiology, Department of Medical Microbiology)

\section{Members of the Examination Board}

Reviewer: Prof. Dr. Uwe Groß

(Institute for Medical Microbiology, Department of Medical Microbiology)

Second reviewer: Prof. Dr. Ernst A. Wimmer

(Johann-Friedrich-Blumenbach-Institute of Zoology and Anthropology,

Department of Developmental Biology)

Further members of the Examination Board:

1. Prof. Dr. Stefanie Pöggeler

(Department of Genetics of Eukaryotic Microorganisms)

2. Prof. Dr. Rolf Daniel

(Department of Genomic and Applied Microbiology)

3. Prof. Dr. Sigrid Hoyer-Fender

(Johann-Friedrich-Blumenbach-Institute of Zoology and Anthropology, Department of Developmental Biology)

4. PD Dr. Stefan Irniger

(Department of Molecular Microbiology and Genetics)

Date of the oral examination: $16^{\text {th }}$ April 2013. 
Printing cost is kindly covered by German Academic Exchange Service/Deutscher Akademischer Austausch Dienst (DAAD). 
"A theory can be proved by experiment but no path leads from experiment to the birth of a theory." 


\section{Declaration}

I hereby declare that this submission entitled "Molecular and Functional Characterization of Programmed Cell Death (PCD) in Toxoplasma gondii“" is my own work and that, to the best of my knowledge and belief, it contains no material previously published or written by another person nor material which to a substantial extent has been accepted for the award of any other degree or diploma of the university or other institute of higher education.

Göttingen, $12^{\text {th }}$ March 2013

Ni Nyoman Ayu Dewi 


\section{Acknowledgements}

Doing experiments are never solitary efforts; therefore I would like to thank everyone who helped in the completion of this project.

I would like to acknowledge and extend my heartfelt gratitude to Prof. Uwe Groß for giving me the opportunity to work in the Insitute for Medical Microbiology, for accepting to be my first referee for my $\mathrm{PhD}$ thesis, for his guidance and constructive advices during my doctoral study.

I would like to express my deep gratitude to Prof. Carsten G.K. Lüder for allowing me to work in his group, for designing a very intriguing and challenging project, and for invaluable discussion, critical advices, encouragement and limitless support throughout my study. He never accepted less than my best efforts.

I would like to thank Prof. Ernst A. Wimmer for accepting to be the second referee for my $\mathrm{PhD}$ thesis and for his critical advices.

I would like also to thank Prof. Stefanie Pöggeler, Prof. Rolf Daniel, Prof. Sigrid HoyerFender, and PD Dr. Stefan Irniger for agreeing to be members of the examination committee for my $\mathrm{PhD}$ disputation.

My special thanks go to Prof. Antonio Jiménez-Ruiz (Madrid) for cooperation in providing information on the structure of TgEndoG and for invaluable discussion, Prof. Dominique Soldati-Favre (Geneva) for providing plasmids containing T. gondii Bax inhibitors sequences and Prof. Dirk Schlüter (Magdeburg) for providing T. gondii tissue cysts to develop chronic infection in mice.

I would like to express my gratitude to Dr. Wolfgang Bohne and Martin Thiele for their collaboration and great assistance on generation of YFP-tagging TgEndoG and also for sharing experiences and stimulating discussion. 
I would like to express my special gratitude to the German Academic Exchange Service (DAAD) for the financial and non-financial support during my study and also to Ms Nina Giebel (International Students Advisor) for her guidance and great assistance on administrative procedures throughout my academic program.

To COST Action BM0802 "Life and death of protozoan parasites", I would like to thank for giving me grants to attend bioinformatics workshop and several scientific meetings abroad; I met many experts who are working on parasites and cell death and I have had very interesting discussions with them.

Many thanks are expressed to my colleagues in Department of Biochemistry Faculty of Medicine, Udayana University, especially to Desak Made Wihandani for her motivation, support and assistance with many bureaucratic procedures.

Special thanks are given to all people in the laboratory. I would like to thank Dr. Raimond Lugert and Dr. Oliver Bader for stimulating discussion. Thanks to Anna, Kristin, San San, Malik, Ichsan and Andrea for being my friends and for all supports. Most especially to Izabela, Jana, Marut, and Maisalreem for telling me things that I do not want to tell myself. And also many thanks to Julia, Maiko and Florian, students who performed lab rotation and helped me to work on T. gondii Bax inhibitors.

Last but not least, most especially, I owe my deepest gratitude to my parents who passed away during my doctoral study period, to my superb husband Aryana Mahayasa and my little princess Ayulia for their limitless support and encouragement, and to whole my family, especially my parents-in-law, for their great support and motivation. Words alone cannot express what I owe them for their encouragement and love. To all of them, I dedicate my work with respect and gratitude. 


\section{Table of Contents}

\section{Declaration}

Acknowledgements

Table of contents

List of Figures

List of Tables

List of Abbreviations

\section{CHAPTER 1}

\section{INTRODUCTION}

1.1. Toxoplasma gondii

1.1.1. A brief history

1.1.2. Medical importance and therapy

1.1.3. Morphology of $T$. gondii

1.1.4. Life cycle and transmission of $T$. gondii infection

1.1.5. Genetics of $T$. gondii

\subsection{Programmed cell death (PCD)}

1.2.1. Classification of cell death

1.2.2. Apoptosis in multicellular organisms 10

1.2.3. Apoptosis-like cell death in unicellular organisms 12

1.3. Objectives of the study

CHAPTER 2

\section{MATERIALS AND METHODS}

\subsection{Materials}

2.1.1. Cell lines, $T$. gondii and bacteria strains 16

2.1.2. Cell death inducers 16

2.1.3. Antibiotics and parasiticidal agents 17

$\begin{array}{ll}\text { 2.1.4. Protease substrate and protease inhibitors } & 17\end{array}$ 
2.1.6. Fluorescent probes 18

2.1.7. Oligonucleotides 18

2.1.8. Plasmids 20

2.1.9. Molecular weight markers 20

2.1.10. Enzymes 21

2.1.11. Kits 21

2.1.12. Other chemicals and reagents 21

2.1.13. Instruments 22

\subsection{Methods}

2.2.1. Maintenance of cells and cell harvest

2.2.1.1. In vitro cultivation of Toxoplasma gondii 23

2.2.1.2. Production of brain cysts 24

2.2.1.3. Cultivation of wild type and transfected cell lines 24

2.2.1.4. Harvesting of T. gondii tachyzoites 25

2.2.1.5. Harvesting mammalian cells 25

2.2.1.6. Cell count 25

2.2.1.7. Cryopreservation of cells 25

2.2.2. Determination of apoptosis markers

2.2.2.1. DNA strand breaks and cell size reduction in extracellular parasites 26

2.2.2.2. DNA strand breaks in intracellular parasites 27

2.2.2.3. Detection of phosphatidylserine exposure 28

2.2.2.4. Effect of pro-apoptotic stimuli on parasite invasiveness 28

2.2.3. Physiological significance of apoptosis-like cell death in $T$. gondii

2.2.3.1. TUNEL staining of T. gondii from the peritoneal cavity during acute 29 toxoplasmosis in mice

2.2.3.2. TUNEL staining of brain cysts from chronically infected mice 29

2.2.3.3. Apoptosis-like cell death after treatment of parasite with 29 chemotherapeutic agents 
2.2.4. Putative regulators of apoptosis-like cell death in $T$. gondii

2.2.4.1. RNA isolation and cDNA synthesis 30

2.2.4.2. Primer design and quantitative real-time PCR 31

2.2.4.3. Heterologous expression of Toxoplasma gondii Bax inhibitors (TgBIs)

2.2.4.3.1. Transfection of TgBIs-coding genes into HeLa cells 31

2.2.4.3.2. Indirect immunofluorescence assay 33

2.2.4.3.3. Colocalisation of TgBIs 33

2.2.4.3.4. Measurement of caspase 3/7 activity 34

2.2.4.3.5. Quantification of protein concentration 35

2.2.4.3.6. Analysis of hypoploid DNA 35

2.2.4.3.7. Redistribution of TgBIs during cell death induction in transgenic HeLa 36 cells

2.2.4.4. Putative T. gondii Endonuclease G (TgEndoG)

2.2.4.4.1. Cloning procedure 36

2.2.4.4.1.1. Digestion of plasmid or PCR products 36

2.2.4.4.1.2. Analysing DNA fragments by agarose gel electrophoresis 37

2.2.4.4.1.3. DNA extraction from agarose gels 37

2.2.4.4.1.4. Vector dephosphorylation 38

2.2.4.4.1.5. Ligation 38

2.2.4.4.1.6. Transformation 38

2.2.4.4.1.7. Screening transformants 39

2.2.4.4.1.8. Plasmid extraction 39

2.2.4.4.1.9. Preparation of glycerol stocks of bacteria 40

2.2.4.4.2. Protein expression 40

2.2.4.4.3. Analysis of protein expression

2.2.4.4.3.1. Sodium Dodecyl Sulphate-Polyacrylamide Gel Electrophoresis $\quad 40$ (SDS-PAGE)

2.2.4.4.3.2. Coomassie blue staining of proteins separated by SDS-PAGE 41

2.2.4.4.3.3. Electroblotting of proteins separated by SDS-PAGE 42

2.2.4.4.3.4. Blocking, washing and antibody incubation 42

2.2.4.4.3.5. Protein detection 44

2.2.5. Regulation of Toxoplasma PCD

2.2.5.1. Detection of $\Delta \Psi \mathrm{m}$ 
2.2.5.2. In situ measurement of caspase-like activity

2.2.5.3. Impact of proteases during induction of apoptosis-like cell death in $T$. gondii

2.2.6. Bioinformatics

2.2.7. Statistical analysis

\section{CHAPTER 3 RESULTS}

3.1. Toxoplasma gondii exhibited markers of apoptosis in response to pro-apoptotic stimuli

3.1.1. The occurrence of DNA fragmentation in $T$. gondii after stimulation of cell death

3.1.2. Cell size reduction in extracellular parasites after treatment with proapoptotic stimuli

3.1.3. Treatment with staurosporine, but not HePC, triggered TUNEL-positivity in intracellular parasites

3.1.4. T. gondii displayed phosphatidylserine externalization following treatment with pro-apoptotic stimuli

3.1.5. Pre-treatment of extracellular parasites with pro-apoptotic stimuli diminished parasite invasion

\subsection{Physiological significance of apoptosis-like cell death in $T$. gondii}

3.2.1. TUNEL- positive parasites were observed during chronic infection with T. gondii, but not during acute infection

3.2.2. An apoptosis-like cell death occurred after treatment of tachyzoites with chemotherapeutic drugs commonly used against toxoplasmosis

3.3. Molecules that may be responsible for the regulation of apoptosis-like cell death in $T$. gondii

3.3.1. Up-regulation of cell death-associated genes following treatment of $T$. gondii with pro-apoptotic stimuli

3.3.2. Toxoplasma gondii Bax inhibitors (TgBIs) 
3.3.2.1. Bioinformatic analysis

3.3.2.2. Immunofluorescence assay of $T$. gondii Bax inhibitors demonstrated distinct localisations of the proteins

3.3.2.3. TgBIs showed a non-mitochondrial localisation 67

3.3.2.4. Redistribution of TgBIs upon cell death induction

3.3.2.5. TgBIs in HeLa cells partially inhibited caspase $3 / 7$ activity following cell death induction

3.3.2.6. TgBIs in HeLa cells reduced the induction of hypoploid DNA following pro-apoptotic treatments

\subsubsection{Toxoplasma gondii Endonuclease G (TgEndoG)}

3.3.3.1. Bioinformatic analysis 73

3.3.3.2. T. gondii Endonuclease $\mathrm{G}$ had a non-mitochondrial localisation 75

3.3.3.3. Cloning and expression T. gondii Endonuclease G

\subsection{Regulation of apoptosis-like cell death in $T$. gondii}

3.4.1. Disruption of mitochondrial membrane potential following treatment of 78 extracellular parasites with pro-apoptotic stimuli

3.4.2. Activation of caspase-like proteases was detected in T. gondii after treatment with pro-apoptotic stimuli

3.4.3. Protease inhibitors prevented miltefosine-triggered cell death in 80 extracellular parasites

\section{CHAPTER 4 DISCUSSION}

4.1. Cell death markers identified in $T$. gondii are characteristic for apoptosis in metazoans

4.2. Possible benefits of an apoptosis-like cell death in $T$. gondii

4.3. Identification of putative regulators of an apoptosis-like cell death in $T$. gondii

4.4. The existence of distinct pathways of an apoptosis-like cell death in $T$. gondii 
Summary 96

References

100

Publications

108

Curriculum vitae

110

Page |ix 


\section{List of Figures}

\section{CHAPTER 1 INTRODUCTION}

Figure 1.1. Schematic structure of tachyzoites and bradyzoites of Toxoplasma gondii

Figure 1.2. Life cycle of Toxoplasma gondii

Figure 1.3. Genomes of Toxoplasma gondii 6

Figure 1.4. Apoptosis pathways in mammalian cells 11

\section{CHAPTER 3 RESULTS}

Figure 3.1. DNA fragmentation in extracellular parasites following treatment with pro-apoptotic stimuli was detected by TUNEL assay followed by flow cytometry

Figure 3.2. Kinetics of DNA fragmentation in T. gondii upon cell death induction by staurosporine or miltefosine as detected by TUNEL assay and flow cytometry

Figure 3.3. Reduction of the parasite cell size and alterations of the morphology of

T. gondii following treatment with pro-apoptotic stimuli were assessed by flow cytometry and immunofluorescence microscopy

Figure 3.4. DNA fragmentation in intracellular parasites after treatment with staurosporine

Figure 3.5. Translocation of phosphatidylserine (PS) after staurosporine or miltefosine treatment

Figure 3.6. Pre-treatment of extracellular parasites with pro-apoptotic stimuli abrogate the ability of $T$. gondii to invade HFF cells

Figure 3.7. TUNEL-positive bradyzoites were detected during chronic infection of T. gondii

Figure 3.8. Induction of an apoptosis-like cell death in extracellular and intracellular $T$. gondii under drug treatments 
Figure 3.9. Transcript levels of $T$. gondii putative PCD-associated genes after treatment of parasites with pro-apoptotic stimuli

Figure 3.10. Expression and localisation of TgBIs in HeLa cells was detected by immunofluorescence microscopy

Figure 3.11. Heterologous expression of TgBIs showed a non-mitochondrial localisation as detected by MitoTracker and anti-FLAG co-staining

Figure 3.12. Redistribution of TgBI-2 and TgBI-3 following staurosporine or brefeldin A treatment

Figure 3.13. Measurement of caspase $3 / 7$ activity partially revealed an inhibitory effect of TgBI-1 and TgBI-2 in HeLa cells, but not TgBI-3, on apoptosis induction in HeLa cells after treatment with staurosporine and brefeldin $\mathrm{A}$

Figure 3.14. Inhibition of hypoploid nuclei by TgBIs upon cell death stimulation in HeLa cells

Figure 3.15. Alignment of EndoG sequences from different species and prediction of TgEndoG structure

Figure 3.16. YFP-tagging of endogenous TgEndoG demonstrated that TgEndoG has a non-mitochondrial localisation

Figure 3.17. Recombinant plasmid construct of pASK-IBA3plus containing TgEndoG coding sequence and heterologous expression of the fusion protein as detected by immunoblotting

Figure 3.18. Dissipation of mitochondrial membrane potential after treatment of extracellular parasites with pro-apoptotic stimuli

Figure 3.19. Caspase-like proteases were activated in $T$. gondii following proapoptotic treatment

Figure 3.20. DNA strand breaks in T. gondii after treatment with miltefosine was inhibited by the presence of protease inhibitors 


\section{List of Tables}

\section{CHAPTER 1 INTRODUCTION}

Table 1.1. Classification of cell death modalities

\section{CHAPTER 2 MATERIAL AND METHODS}

Table 2.1. Cell death inducers and concentrations used in this study 16

Table 2.2. Antibiotics and antiparasite drugs and concentrations used in this 17 study

Table 2.3. Protease substrate and protease inhibitors and concentrations used in this study

Table 2.4. Antibodies used for Western Blot (WB) or immunofluorescence assay (IFA) and concentrations used in this study

Table 2.5. Primer pairs for amplifying cell death-related genes via quantitative real time PCR

Table 2.6. Primer pairs for cloning and sequencing of T. gondii

Endonuclease G

\section{CHAPTER 3 RESULTS}

Table 3.1. Toxoplasma gondii cell death-associated genes

Table 3.2. Characteristic of TgBIs

\section{CHAPTER 4 DISCUSSION}

Table 4.1. Regulation of an apoptosis-like cell death in T. gondii following staurosporine and miltefosine treatment 


\section{List of Abbreviations}

AIDS

AIF

APS

ATc

$\mathrm{Bcl}$

BI-1

BLAST

BSA

cDNA

$\mathrm{ddH}_{2} \mathrm{O}$

$\Delta \Psi \mathrm{m}$

DEVD-AMC

DMEM

DMSO

DNA

dNTP

DTT

ECL

E. coli

EDTA

EndoG

ER

E64

FACS

FCS

g

h

$\mathrm{HePC}$

HFF

HRP
Acquired immunodeficiency syndrome

Apoptosis-inducing factor

Ammonium persulfate

Anhydrotetracycline

B-cell lymphoma

Bax inhibitor-1

Basic local alignment search tool

Bovine serum albumin

Complementary DNA

Double distilled water

Mitochondrial membrane potential

Asp-Glu-Val-Asp-Aminomethylcoumarin

Dulbecco's modified eagle medium

Dimethyl sulfoxide

Deoxyribonucleic acid

Dinucleotide phosphate

1,4-Dithiothreitol

Enhanced chemiluminescent detection

Escherichia coli

Ethylene diamine tetraacetic acid

Endonuclease G

Endoplasmic reticulum

trans-Epoxysuccinyl-L-leucylamido(4-guanidino)butane

Fluorescence activated cell sorting

Fetal calf serum

Force of gravity

Hour(s)

Hexadecylphosphorylcholine (miltefosine)

Human foreskin fibroblast

Horseradish peroxidase 


\begin{tabular}{|c|c|}
\hline IFA & Immunoflurescence assay \\
\hline $\operatorname{IgG}$ & Immunoglobulin $\mathrm{G}$ \\
\hline IPTG & Isopropyl beta-D-thiogalactopyranoside \\
\hline $\mathrm{kb}$ & Kilo base pair \\
\hline $\mathrm{kDa}$ & Kilo Dalton \\
\hline LB & Luria Broth \\
\hline M & Molar \\
\hline $\mathrm{ml}$ & Milliliter \\
\hline $\min$ & Minute \\
\hline $\mathrm{mg}$ & Milligram \\
\hline$\mu \mathrm{g}$ & Microgram \\
\hline$\mu l$ & Microliter \\
\hline$\mu \mathrm{m}$ & Micrometer \\
\hline MOMP & Mitochondrial outer membrane permeabilisation \\
\hline mRNA & Messenger RNA \\
\hline NEAA & Non-essential amino acid \\
\hline $\mathrm{nM}$ & nanoMolar \\
\hline$p$ & $P$ value \\
\hline PAGE & Polyacrylamide gel electrophoresis \\
\hline PBS & Phosphate buffered saline \\
\hline PCD & Programmed cell death \\
\hline PCR & Polymerase chain reaction \\
\hline PE & Phycoerythine \\
\hline PV & Parasitophorous vacuole \\
\hline RNA & Ribonucleic acid \\
\hline RNase & Ribonuclease \\
\hline $\mathrm{rpm}$ & Revolutions per minute \\
\hline RPMI & Roswell Park Memorial Institute \\
\hline RT & Room temperature \\
\hline RT-PCR & Reverse transcription-polymerase chain reaction \\
\hline $\sec$ & Second \\
\hline SDS & Sodium dodecyl sulphate \\
\hline SEM & Standard error of the mean \\
\hline
\end{tabular}


7-AAD

Stau

tet

$\mathrm{Tg}$

Tm

Tris

tRNA

TUNEL

U

UV

$\mathrm{V}$

V

W

WB

WT

YFP

Z-VAD-FMK
7-Amino-Actinomycin D

Staurosporine

Tetracycline

T. gondii

Annealing temperature

Tris(hydroxylmethyl)-aminomethan

transfer RNA

Terminal deoxynucleotidyl transferase-mediated X-dUTP nick end labelling

Unit

Ultraviolet

Voltage

Volume

Weight

Western blot

Wildtype

Yellow fluorescence protein

z-Val-Ala-Asp-fluoromethylketone 


\section{CHAPTER 1 INTRODUCTION}

\subsection{Toxoplasma gondii}

\subsubsection{A brief history}

Nicolle and Manceaux, scientists who were working in North Africa, unintentionally discovered a protozoan in tissues of a hamster-like rodent, Ctenodactilus gundi, in 1908. The parasite was later named Toxoplasma gondii based on the morphology and the host (in Greek: toxo $=$ arc or bow, plasma $=$ life). Another scientist in Brazil, Alfonso Splendore, also discovered the same parasite in a rabbit in 1908 (Dubey, 2008; Innes, 2010). However, it took then 60 years to unravel the complex life cycle of $T$. gondii (Frenkel et al., 1970).

Toxoplasma gondii is an obligate intracellular protozoan parasite and can infect all warm-blooded animals including humans (Innes, 2010). It belongs to the phylum Apicomplexa that consist of more than 5000 species of parasitic protozoa. Many apicomplexan parasites are important pathogens of humans and animals including Plasmodium (responsible for malaria), Eimeria (a poultry and cattle pathogen), Neospora (a pathogen of dogs and cattle), Cryptosporidium (an opportunistic human and animal pathogen, devastating for AIDS patients), Babesia and Theileria (cattle parasites) (Hu et al., 2004; Saeij et al., 2005).

\subsubsection{Medical importance and therapy}

Toxoplasma gondii infection is highly prevalent in humans and animals worldwide with varying prevalence in different geographic areas (Dubey, 1998; Hill and Dubey, 2002). For example, in the United States and the United Kingdom, an estimated 16 to $40 \%$ of the population is infected. In Central and South America and parts of Europe, 50 to 80 $\%$ of humans are estimated to be infected (Hill and Dubey, 2002). Infection of T. gondii is typically asymptomatic; however it may lead to severe symptoms or be even fatal (death) in individuals with immune dysfunction, e.g. AIDS patients and in developing foetuses and 
neonates. Additionally, this parasite was more recently found to be related with severe intraocular inflammation after infection of immunocompetent adults (Innes, 2010).

Several chemotherapeutic drugs are available to treat symptomatic toxoplasmosis or to prevent the development of clinical manifestation. A drug regimen that is widely used up to now for treating toxoplasmosis is a combination of sulphadiazine, pyrimethamine and folinic acid (Montoya and Remington, 2008). Other drugs that are also commonly used include atovaquone, spiramycin, clindamycin, or diaminodiphenylsulphone (Hill and Dubey, 2002). However, drugs currently available are not effective to eradicate the parasite, particularly the persistent bradyzoite stage. Instead, they only target the tachyzoite stage of $T$. gondii, which is however responsible for the clinical symptoms during acute toxoplasmosis. Side effects of the drugs have been also reported; for instance, spiramycin has a teratogenic effect and thus can not be used to treat toxoplasmosis during pregnancy (Montoya and Remington, 2008). Therefore, new approaches to design novel drugs to eradicate $T$. gondii are required.

\subsubsection{Morphology of $T$. gondii}

Toxoplasma gondii has three infectious stages: tachyzoites, bradyzoites, and sporozoites (Dubey et al., 1998).

The tachyzoite is the rapidly multiplying intracellular stage which is associated with acute infection. The term tachyzoite was invented by Frenkel in 1973 (tachos $=$ speed in Greek). Before that, this stage was called trophozoite or endozoite. It has a crescent shape, approximately 2 by $6 \mu \mathrm{m}$, with a pointed anterior (conoidal) end and a rounded posterior end (Figure 1.1). The shape of tachyzoites becomes ovoid after they enter the host cells by actively penetrating through the host cell plasma membrane. Within the host cells, tachyzoites are enclosed within a parasitophorous vacuole (PV), the membrane of which is formed by both the parasite and the host cell. Tachyzoites multiply asexually within the host cell by endodyogeny until the host cell ruptures because it can no longer support the growth of tachyzoites. After dissemination within their hosts, tachyzoites differentiate into bradyzoites in the brain, muscles, and other organs, where they may remain latent for the host's life (Dubey et al., 1998; Dzierszinski et al., 2004). 
The bradyzoite, a slowly growing parasite stage (brady = slow), is the stage present in tissue cysts. Bradyzoites are also named cystozoites (Dubey, 2008; Dubey et al., 1998; Weiss and Kim, 2000). Bradyzoites divide by endodyogeny in tissue cysts and also remain intracellular. The size of tissue cysts is variable; young tissue cysts may contain only two bradyzoites and the diameter may be as small as $5 \mu \mathrm{m}$, while older cysts may contain hundreds of bradyzoites. The crescent-shaped bradyzoite has approximately 7 by $1.5 \mu \mathrm{m}$ in size (Figure 1.1) (Dubey et al., 1998).

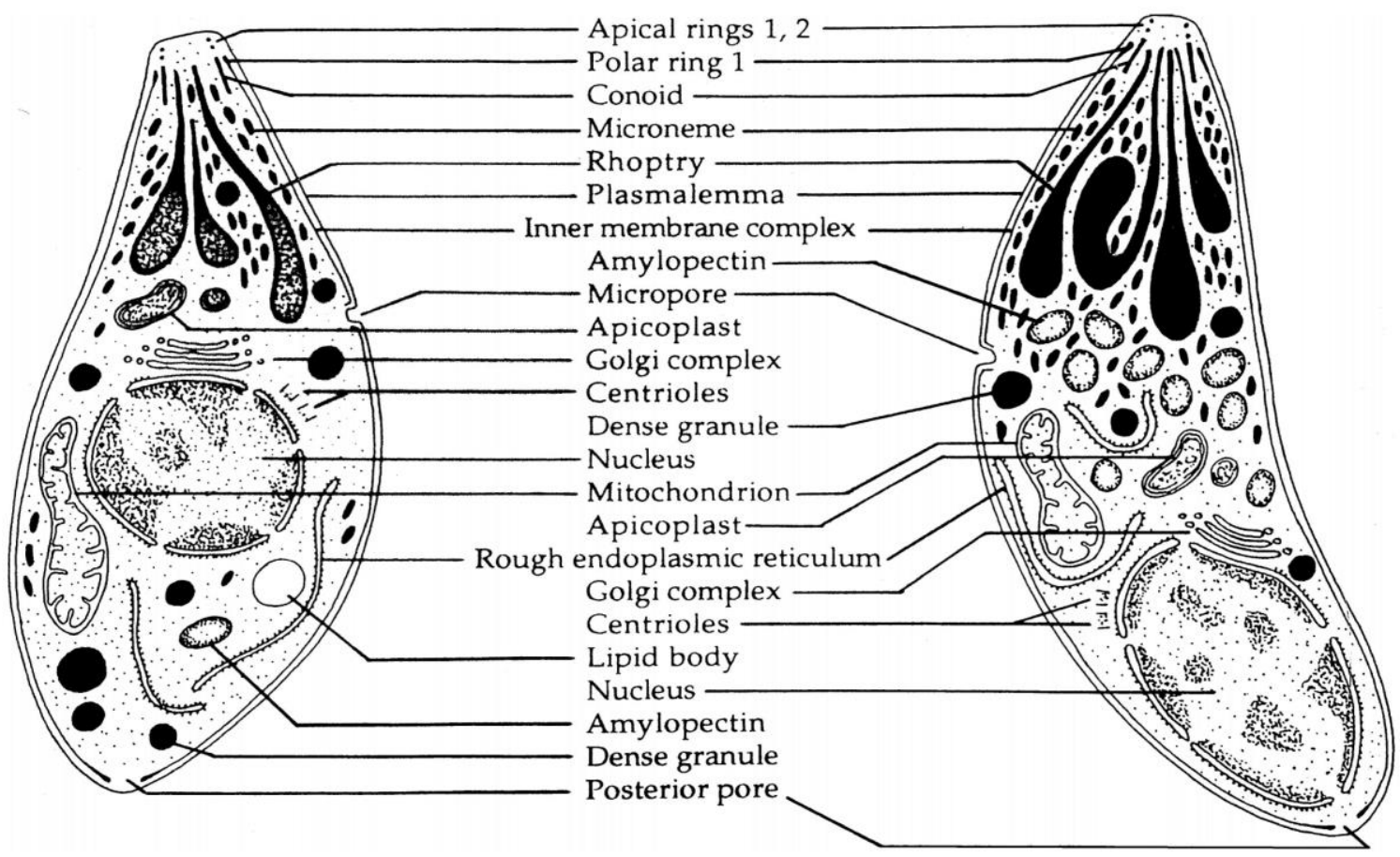

Figure 1.1. Schematic structure of tachyzoites (left) and bradyzoites (right) of Toxoplasma gondii. Tachyzoites and bradyzoites of $T$. gondii are structurally similar, however they vary in certain organelles and inclusion bodies. For instance, the nucleus of tachyzoites is located in the centre of the parasite while in bradyzoites, the nucleus is situated towards the posterior end (Dubey et al., 1998). Picture is taken from Dubey et al. (1998), p. 269.

Oocysts are only shed by the definitive hosts, i.e. members of the Felidae, particularly the cat, after ingesting any of the three infectious stages. The shortest prepatent period, i.e. the time from infection until the shedding of oocysts, is after ingestion of tissue cysts ( 3 to 10 days). The prepatent period is 13 days or more after consuming tachyzoites and 18 days or more after oocysts ingestion (Dubey, 2008; Dubey et al., 1998). Almost all 
cats shed oocysts after tissue cysts ingestion whereas less than 30-50\% of cats shed oocysts after ingestion of tachyzoites or oocysts (Dubey, 2009; Dubey et al., 1998). Unsporulated oocyts are 10 by $12 \mu \mathrm{m}$ in diameter and have a subspheric to spheric shape. Sporulation occurs in the environment after 1 to 5 days of excretion, depending on aeration and temperature. Sporulated oocysts are subspheric to ellipsoid, 11 by $13 \mu \mathrm{m}$ in diameter. Each oocyst contains 2 ellipsoidal sporocysts; each sporocyst is 6 by $8 \mu \mathrm{m}$ in size and contains 4 sporozoites. Sporozoites are morphologically similar with tachyzoites with a size of 2 by 6 to $8 \mu \mathrm{m}$ and have a subterminal nucleus (Dubey et al., 1998).

\subsubsection{Life cycle and transmission of $T$. gondii infection}

Toxoplasma gondii has a complex life cycle; it consists of a sexual cycle in its definitive hosts and asexual cycles both in its definitive and intermediate hosts (including humans) (Figure 1.2). So far, the development of T. gondii in cats has only been studied in detail following ingestion of tissue cysts (Dubey, 1998). After ingestion of tissue cysts, the cyst wall is digested and bradyzoites are released into the intestinal lumen and then cross the intestinal wall. The parasites replicate throughout the body as rapidly dividing tachyzoites (extra-intestinal cycle). Simultaneously, the organisms invade and replicate within the intestinal epithelial cells (entero-epithelial cycle). During the entero-epithelial cycle, five distinct morphological asexual types (A-E) are formed in the enterocytes of cats, leading to a sexual cycle (which only occurs in the definitive host) and finally resulting in the production of oocysts (Dubey, 1998; Dubey et al., 1998). Oocysts are secreted within the cats' feces into the environment, where they undergo sporulation and contaminate food, water and soil. As the cat develops an immune response, cats stop shedding oocyst and the development of tachyzoites is also halted. Bradyzoites (slowly replicating forms of the organism) within tissue cysts are subsequently formed. Cats continue shedding million of oocysts for 1-2 weeks. Once cats develop an immune response to $T$. gondii, reshedding of oocysts is extremely rare (Dubey, 1995).

Intermediate hosts can be infected by the ingestion of sporulated oocysts. As in the cats, parasites undergo an extra-intestinal cycle (asexual reproduction). Subsequent immune response leads to the development of encysted bradyzoites which may persist for years (potentially for the host's lifetime), mainly in the nervous system, muscles and heart 
tissue (Dubey et al., 1998). The tissue cysts containing bradyzoites are infectious to cats and also to other intermediate hosts (Innes, 2010). Therefore, a regular transmission route is also via ingestion of tissue cysts in infected undercooked or cured meat.

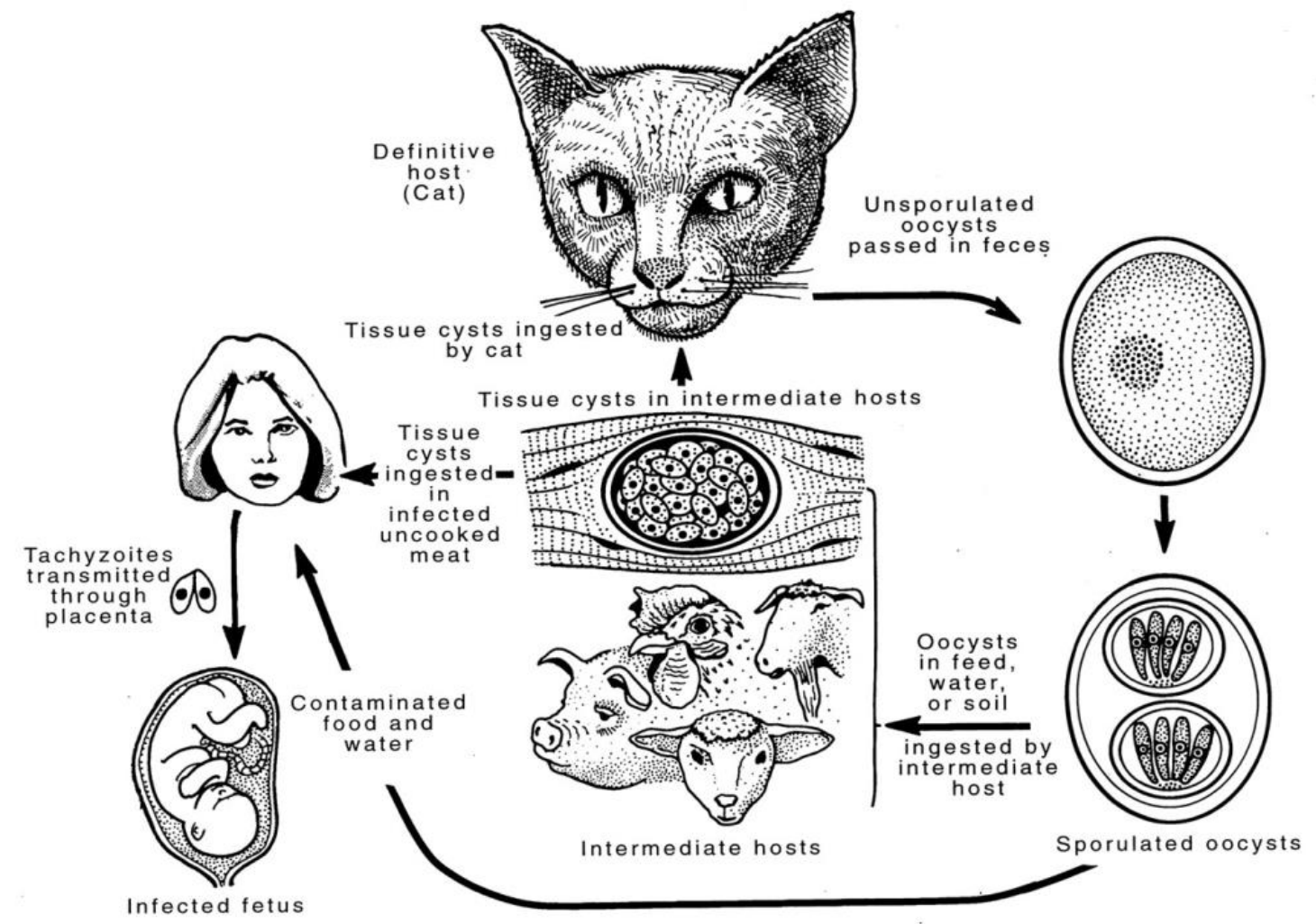

Figure 1.2. Life cycle of Toxoplasma gondii.

T. gondii undergoes its sexual cycle only in the intestine of Felidae, mostly cats (definitive host). The definitive host shed oocysts that undergo sporogony in the environment to generate sporozoites. Oocysts are environmentally resistant and can infect a variety of intermediate hosts. Acute infection is characterised by dissemination of tachyzoites within the host. Primary infection in pregnant women can lead to transmission of infection to the foetus, possibly resulting in miscarriage or severe birth defects. Tissue cysts harbouring bradyzoites are responsible for long-term infection and are transmitted following oral ingestion by intermediate hosts (Dubey et al., 1998; Sibley et al., 2009). Picture is taken from Dubey et al. (1998), p. 268.

Additionally, transmission of infection may also be acquired by a vertical transmission route (congenital transmission) because tachyzoites are able to across the placenta. In humans, transmission to the foetus occurs mostly when pregnant women get a primary infection during gestation (Montoya and Remington, 2008). 


\subsubsection{Genetics of $T$. gondii}

Strains of $T$. gondii are divided into three main clonal groups based on polymorphic markers and on virulence in laboratory mice. Remarkably, these clonotypes differ at the genetic level by only 1 to $2 \%$ (Saeij et al., 2005; Su et al., 2003). Strains of the type I lineage are highly virulent in mice with a LD100 of less than 10 parasites whereas strains of the type II and III lineages are less virulent in mice with LD100 of more than 1000 parasites (Kim and Weiss, 2004). Toxoplasma gondii has a nuclear genome consisting of 14 linear chromosomes (around $65 \mathrm{Mb}$ ), a mitochondrial genome containing $6 \mathrm{~kb}$ tandem repeats and a $35 \mathrm{~kb}$ apicoplast genome (Figure 1.3) (Sibley and Ajioka, 2008). Genome sequences and annotations of strain ME49 (type II) of $T$. gondii as well as genomic sequence of strain GT1 (type I), VEG (type III) and chromosomes Ia and Ib of RH strain (type I) can be accessed via www.toxodb.org (Gajria et al., 2008).

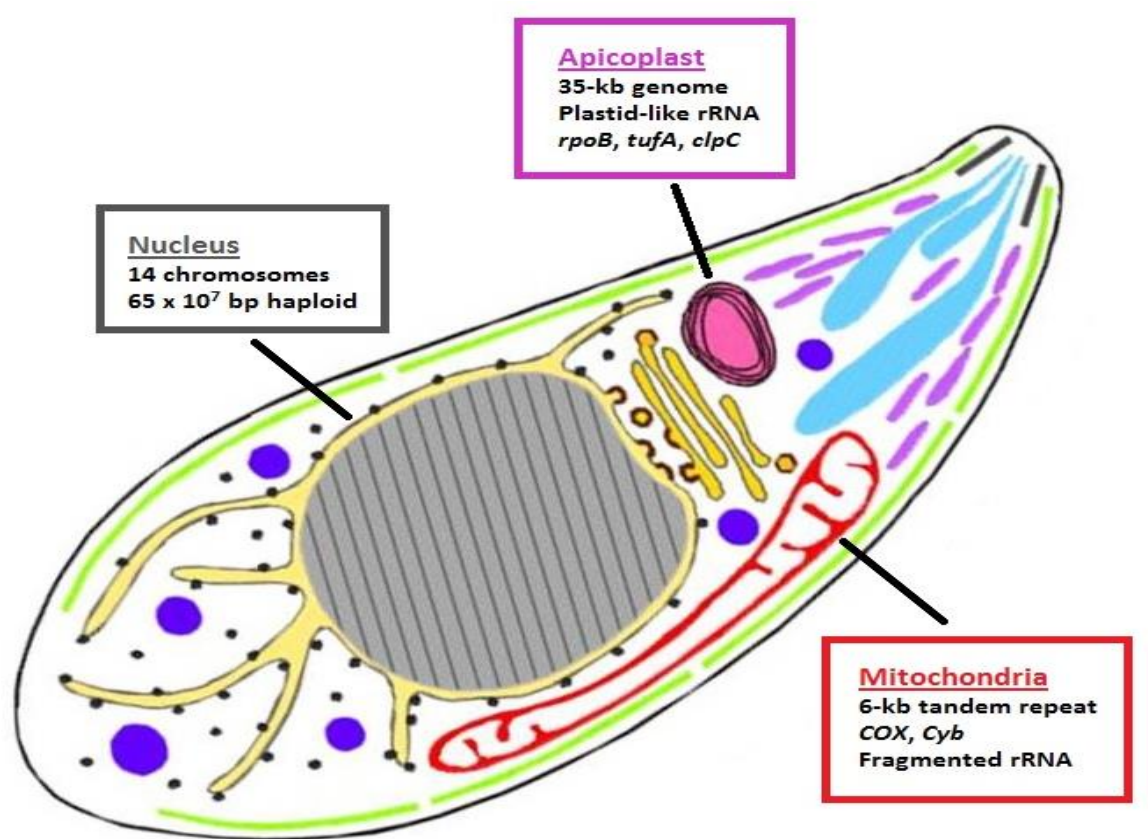

Figure 1.3. Genomes of Toxoplasma gondii.

Nucleus, mitochondria, and apicoplast are organelles that contribute to the total genome of T. gondii (picture is adapted from Nishi et al., 2008; Sibley and Ajioka, 2008). The nuclear genome is composed of 14 linear chromosomes and around $65 \mathrm{Mb}$ in size. The $6 \mathrm{~kb}$ tandem repeats of the mitochondrial genome encodes genes for cytochrome $\mathrm{c}$ oxidase subunits, cytochrome $\mathrm{b}$ and fragmented ribosomal RNAs and the $35 \mathrm{~kb}$ circular apicoplast genome encodes only a few genes encoding proteins including tandemly duplicated ribosomal RNAs and tRNAs (Sibley and Ajioka, 2008). 


\subsection{Programmed Cell Death (PCD)}

\subsubsection{Classification of cell death}

Classification of cell death was initially made based on morphological characteristics. With progress in biochemical and genetic assays for the assignment of cell death-related phenomena, the Nomenclature Committee on Cell Death (NCCD) recommended unified criteria for the definition of cell death which are based on different cell death morphologies and biochemical features (Galluzzi et al., 2012; Kroemer et al., 2009). This should facilitate the use of the same terminology by different scientists. According to the revised NCCD recommendations (Galluzzi et al., 2012), cell death can be functionally classified into extrinsic apoptosis, caspase-dependent or -independent intrinsic apoptosis, regulated necrosis, autophagic cell death and mitotic catastrophe.

Definitions of each type of cell death according to the latest NCCD recommendation (Galluzzi et al., 2012) are as follows:

1. Extrinsic apoptosis is defined as a caspase-dependent cell death which is induced by extracellular stress signal. The signal will then be recognised by specific transmembrane death receptors, such as Fas/CD95, TNF $\alpha$ receptor 1 and TRAIL receptor. This mechanism can be suppressed by pan-caspase chemical inhibitors (e.g. Z-VAD-FMK). The criteria of extrinsic apoptosis involve one of three major lethal signalling cascades. First, activation of the caspase 3 cascade following death receptor signalling and activation of caspase 8 . Second, activation of caspase 3 that involves mitochondrial outer membrane permeabilisation (MOMP) which is initiated by translocation of tBID following death receptor signalling and activation of caspase 8. Or, third, caspase 3 that is activated following ligand deprivationinduced dependence receptor signalling.

2. Intrinsic apoptosis is a cell death mechanism in response to various intracellular stress signals such as DNA damage, oxidative stress, accumulation of unfolded proteins in the endoplasmic reticulum (ER stress), etc. This form of cell death is mediated by MOMP and is linked with generalised and irreversible mitochondrial membrane potential disruption, release of proteins from the intermembrane space of mitochondria (e.g. cytochrome c, Smac, Diablo, HtrA2, AIF, EndoG) into the cytosol, and inhibition of the mitochondria respiratory chain. 
There are two subtypes of intrinsic apoptosis: caspase-dependent and caspaseindependent pathways based on a cytoprotective effect of caspase inhibition.

3. Recently, necrosis is believed to at least partially occur in a regulated way, although for many years, it has been described as a cell death process that only occurs by accident (or uncontrolled). Necrosis lacks the features of apoptosis and autophagy. Regulated necrosis can be induced by alkylating DNA damage, excitotoxins and ligation of cell death receptors under certain circumstances.

4. Autophagic cell death is a mechanism that is mediated by autophagy (cells eat themselves). The mechanism can be inhibited by chemicals and/or genetic means. Morphologically, autophagic cell death is characterised by a massive cytoplasmic vacuolisation.

5. Mitotic catastrophe was occasionally used to define cell death occurring in mitosis. Lately, it has been proposed that mitotic catastrophe might not constitute a bona fide cell death executioner mechanism, but an oncosuppresive mechanism. The oncosuppresive process is initiated by disturbation of the mitotic machinery during the $\mathrm{M}$ phase of the cell cycle and coincides to some extent with a mitotic arrest, which finally induces cell death or senescence.

Comparisons between types of cell death, morphologically and biochemically, are summarised in Table 1.1. 
Table 1.1. Classification of cell death modalities

\begin{tabular}{|c|c|c|c|}
\hline $\begin{array}{l}\text { Type of cell } \\
\text { death }\end{array}$ & $\begin{array}{l}\text { Main biochemical } \\
\text { features }\end{array}$ & $\begin{array}{c}\text { Caspase } \\
\text { dependence }\end{array}$ & $\begin{array}{l}\text { Main morphological } \\
\text { features }\end{array}$ \\
\hline $\begin{array}{l}\text { Extrinsic } \\
\text { apoptosis by } \\
\text { death receptor }\end{array}$ & $\begin{array}{l}\text { Death receptor signalling } \\
\text { Caspase } 8 \text { activation } \\
\text { BID cleavage and } \\
\text { MOMP } \\
\text { Caspase } 3(6,7) \\
\text { activation }\end{array}$ & yes & $\begin{array}{l}\text { Pyknosis, chromatin } \\
\text { condensation, plasma } \\
\text { membrane blebbing }\end{array}$ \\
\hline $\begin{array}{l}\text { Caspase- } \\
\text { dependent } \\
\text { intrinsic } \\
\text { apoptosis }\end{array}$ & $\begin{array}{l}\text { MOMP } \\
\text { Irreversible disruption of } \\
\Delta \Psi \mathrm{m}\end{array}$ & yes & $\begin{array}{l}\text { Pyknosis, chromatin } \\
\text { condensation, plasma } \\
\text { membrane blebbing }\end{array}$ \\
\hline $\begin{array}{l}\text { Caspase- } \\
\text { independent } \\
\text { intrinsic } \\
\text { apoptosis }\end{array}$ & $\begin{array}{l}\text { Release of IMS proteins } \\
\text { Inhibition of respiratory } \\
\text { chain }\end{array}$ & no & $\begin{array}{l}\text { Pyknosis, chromatin } \\
\text { condensation, plasma } \\
\text { membrane blebbing }\end{array}$ \\
\hline $\begin{array}{l}\text { Autophagic } \\
\text { cell death }\end{array}$ & $\begin{array}{l}\text { Lipidation of MAP1LC3 } \\
\text { Degradation of SQSTM1 }\end{array}$ & no & Vacuolisation \\
\hline Necrosis & $\begin{array}{l}\text { Death receptor signalling } \\
\text { Inhibition of caspase } \\
\text { Activation of RIP1 } \\
\text { and/or RIP3 }\end{array}$ & no & $\begin{array}{l}\text { Nuclear membrane } \\
\text { dilatation, circumscribed } \\
\text { chromatin condensation, } \\
\text { increased cell volume } \\
\text { (oncosis) }\end{array}$ \\
\hline $\begin{array}{l}\text { Mitotic } \\
\text { catastrophe }\end{array}$ & $\begin{array}{l}\text { Caspase } 2 \text { activation (in } \\
\text { some cases) } \\
\text { TP53 or TP73 activation } \\
\text { (in some cases) } \\
\text { Mitotic arrest }\end{array}$ & no & $\begin{array}{l}\text { Micronucleation and } \\
\text { multinucleation }\end{array}$ \\
\hline
\end{tabular}

(modified from Galluzzi et al., 2012; Vitale et al., 2011).

Abbreviations: $\Delta \Psi \mathrm{m}$, mitochondrial membrane potential; IMS, intermembrane space; MAP1LC3, microtubule-associated protein 1 light chain 3; MOMP, mitochondrial outer membrane permeabilisation; SQSTM1, sequestosome. 


\subsubsection{Apoptosis in multicellular organisms}

Apoptosis is the most frequent phenotype of self-destruction of a cell. The term apoptosis has been originally introduced by Kerr et al. (1972) to define a type of cell death with specific morphological traits. The word "apoptosis" in Greek means "dropping off" or "falling off" of petals from flowers, or leaves from trees (Kerr et al., 1972 ). Apoptosis is an energy-dependent mechanism and a highly regulated process which plays crucial roles in the development of multicellular organisms as well as in the regulation and maintenance of the cell population under physiological and pathological circumstances (Elmore, 2007).

Histologically, apoptosis is characterised by rounding-up of the cells, retraction of pseudopodes, reduction of cellular volume (pyknosis), chromatin condensation, nuclear fragmentation, plasma membrane blebbing and phagocytosis and degradation by other cells (Kerr et al., 1972; Kroemer et al., 2009). Biochemical features of apoptosis include protein cleavage, protein cross-linking, DNA fragmentation, and externalisation of phosphatidylserine to the outer leaflet of the plasma membrane (Elmore, 2007).

Classical apoptosis pathway in vertebrates can be triggered by extrinsic (death receptor) or intrinsic (mitochondrial-dependent) stimuli (see section 1.2.1). The extrinsic pathway is activated by cell surface death receptors (TNF receptor, CD95/Fas, TRAIL death receptors, etc.) and the intrinsic pathway is associated with the formation of the socalled apoptosome, which is formed by Apaf-1, cytochrome c and pro-caspase 9 (Jeong and Seol, 2008; Vaux and Strasser, 1996) (Figure 1.4). Both pathways can converge at the execution phase that is initiated by the cleavage of caspase 3 which then activates proteases and finally leads to degradation of nuclear material and cytoskeletal proteins (Elmore, 2007).

Inevitably, caspases are crucial molecules in apoptosis pathways in metazoans. There are several types of caspases, two of them are involved in mammalian apoptosis: initiator caspases (caspase 2, 8, 9 and 10) and executioner/effector caspases (caspase 3, 6, and 7). Caspases reside within cells in an inactive form and will be activated following triggering of apoptosis. Caspases have different substrate preferences but they always cleave their substrate after aspartate residues (Green, 2011; Shi, 2002).

Although many studies have confirmed the critical importance of caspases in the regulation of apoptosis in metazoa, apoptosis can be executed by a mechanism which 
ensues independently of caspases. Caspase-independent cell death is defined as a form of cell death that occurs when caspases cannot be activated following common stimuli of apoptosis. Nevertheless this type of cell death often shares features as described during caspase-dependent apoptotic cell death (Tait and Green, 2008).

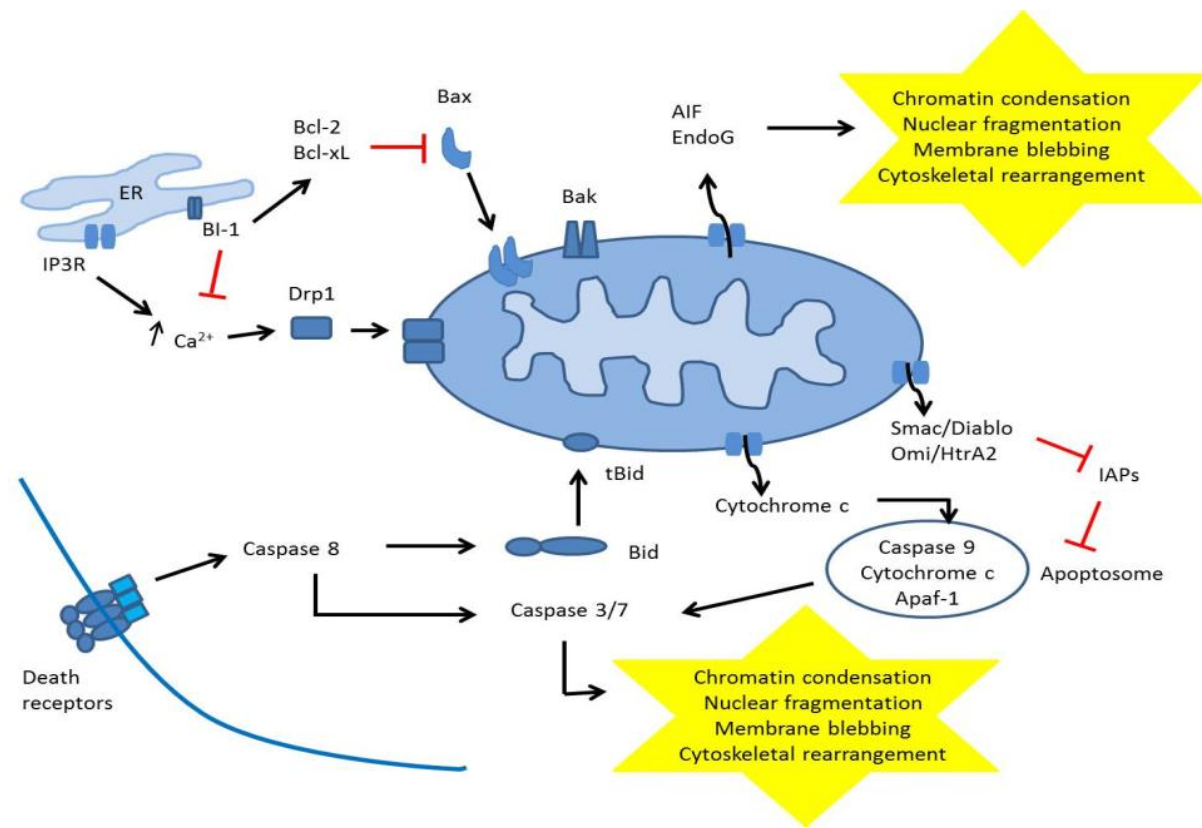

Figure 1.4. Apoptosis pathways in mammalian cells.

The extrinsic apoptosis pathway is initiated by transmembrane death receptor-mediated interactions. This interaction will activate caspase 8 which in turn activates a downstream caspase cascade and leads to cell death. On the other hand, caspase 8 cleaves Bid (BH3-only protein, pro-apoptotic member of Bcl-2 family) and the truncated Bid (tBid) translocates to mitochondria which results in the activation of intrinsic apoptosis pathway. Activation of the intrinsic (mitochondria-dependent) pathway leads to the release of cytochrome c into the cytosol, the formation of the apoptosome and activation of the caspase cascade. Members of Bcl-2 family proteins play important roles in regulation of cytochrome c release. Bax and Bak (pro-apoptotic members of Bcl-2 family) translocate from the cytosol to mitochondrial membrane as homodimers. Antiapoptotic members of Bcl-2 family, Bcl-2 and Bcl-xL, inhibit the function of Bax and Bak. Release of $\mathrm{Ca}^{2+}$ from the ER through the IP3R (inositol 1,4,5-triphosphate receptor) stimulates translocation of the DRP-1 protein to mitochondria. Accumulation of $\mathrm{Ca}^{2+}$ in mitochondria in turn is responsible for the release of cytochrome c, consequently leading to cell death. AIF and EndoG, proteins with nuclease activity, are released from mitochondria and translocated into nucleus. They execute cell death independently of caspases. Mitochondria also release Smac/Diablo and Omi/HtrA2 and they block the activation of inhibitors of apoptosis (IAPs) (adopted from Jeong and Seol, 2008). Bax inhibitor-1, a protein that mainly localises at the ER membrane, acts as an anti-apoptotic regulator by interaction with Bcl-2 or Bcl-xL or by decreasing cytosolic $\mathrm{Ca}^{2+}$ level (Robinson et al., 2011; Xu and Reed, 1998). 
Many proteins (caspases, cathepsins, endonucleases, calpains, and other proteases) play roles in the execution of apoptosis, and they can be regulated by several cellular organelles, including mitochondria and endoplasmic reticulum (ER) (Figure 1.4). These organelles and their specific apoptosis-related proteins may act independently or influence each other (Broker et al., 2005).

In vertebrates, mitochondria are the central players in both caspase-dependent and caspase-independent apoptosis pathways. In caspase-dependent apoptosis pathway, mitochondria play pivotal roles by releasing molecules that induce caspase activation and chromatin condensation (Jeong and Seol, 2008). On the other hand, apoptosis-inducing factor (AIF) and Endonuclease $G$ are released by mitochondria and act in a caspaseindependent fashion to execute apoptosis (Broker et al., 2005; Elmore, 2007; van Loo et al., 2001). The endoplasmic reticulum is also an important organelle in the execution of apoptosis pathways. It regulates apoptosis either by releasing cell death signals of its own as a response to ER stress or by transmitting death-promoting signals to mitochondria following death stimuli (Breckenridge et al., 2003; Hetz, 2007; Madeo and Kroemer, 2009). The mitochondria and ER are linked physiologically, particularly in the regulation of $\mathrm{Ca}^{2+}$ dynamics. $\mathrm{Ca}^{2+}$ released from the ER will be taken up by mitochondria, causing mitochondrial damage and thus leading to cell death (Jaattela and Tschopp, 2003; Scorrano et al., 2003).

\subsubsection{Apoptosis-like cell death in unicellular organisms}

Cell death, e.g. apoptosis, was until recently believed to be confined to multicellular taxa only. Nevertheless, there is increasing evidence that an ancestral form of apoptosis exists in unicellular organisms as well including protozoan parasites e.g. Leishmania spp, Plasmodium spp, Trichomonas vaginalis, Trypanosoma cruzi, Trypanosoma brucei, and Giardia lamblia (Bruchhaus et al., 2007; Jiménez-Ruiz et al., 2010; Reece et al., 2011). Apoptosis-like cell death in protozoan parasites shares characteristic features of apoptosis in metazoa, e.g. phosphatidylserine exposure, DNA fragmentation, chromatin condensation, loss of mitochondrial membrane potential, cell shrinkage, and protease activation (Bruchhaus et al., 2007; Jiménez-Ruiz et al., 2010) . However, the underlying molecular mechanisms of cell death in protozoan parasites obviously differ from those in 
metazoans. For instance, bona fide caspases, i.e. key players of apoptosis in metazoans, are absent in protozoan parasites as are members of the Bcl-2 family (Nedelcu, 2009). Instead, other proteins or molecules may contribute to apoptosis-like cell death in protozoan, such as metacaspases, cathepsins, or calpain (Arnoult et al., 2002; Ch'ng et al., 2010; El-Fadili et al., 2010; Hurd et al., 2006). Considering that protozoan parasites do not express genuine caspases, it seems that apoptosis-like cell death in the parasites proceeds via a caspase-independent pathway.

The existence of an apoptosis-like cell death in single-celled organisms has opened up an intriguing question: what is the benefit of a self-killing mechanism for protozoan parasites? There is now evidence for different functions of programmed cell death pathways in protozoan parasites. PCD could be useful to control cell proliferation, differentiation and survival of Trypanosoma cruzi (Ameisen et al., 1995), for limiting intensity of infection in the host as shown for the malaria parasite, Plasmodium berghei (Al-Olayan et al., 2002), or to evade host immunity in order to establish infection as described for Leishmania (van Zandbergen et al., 2006). PCD arguably plays essential roles in regulating parasites densities in distinct host compartments and in avoiding inflammatory responses to ensure a sustained infection (van Zandbergen et al., 2010).

Studies on elucidating programmed cell death in protozoan parasites are still scarce. Even though apoptosis-like cell death in unicellular organisms resembles apoptosis in metazoans as described above, the mechanisms still remain largely unclear. Studies on $T$. gondii programmed cell death are hitherto limited and consequently, there is only little information available regarding apoptosis or programmed cell death in T. gondii.

When we consider $T$. gondii as a population of genetically identical organisms within its host (and T. gondii tissue cysts as a community of highly inter-dependent organisms possibly communicating between each other), a form of programmed cell death of a subpopulation may be evolutionary favorable and may increase the parasite fitness because it may avoid an overwhelming parasite load and host death. Furthermore, a mechanism known as apoptotic mimicry has been reported in $T$. gondii in order to dampen the host immune response by inducing TGF- $\beta$ secretion (Seabra et al., 2004). Finally, it has also been described that the parasite inhibits NO production by translocating phosphatidylserine (PS) to its outer leaflet of the plasma membrane in order to ensure its 
persistence in activated macrophages (Seabra et al., 2004). Exposure of PS by T. gondii appeared to be also required to maintain parasite and host survival (Santos et al., 2011). Some markers of apoptosis, e.g. chromatin condensation, DNA degradation and hypoploid nuclei, have been described in the tachyzoite stage of $T$. gondii following treatment with the exogenous nitric oxide (NO) donor sodium nitroprusside (Peng et al., 2003).

\subsection{Objectives of the study}

Toxoplasma gondii is one of the most successful parasites that can infect humans and animals, leading to a major health problem worldwide. During recent years, programmed cell death (PCD), a common feature of multicellular organisms that is crucial to the development, homeostasis and integrity of multicellular organisms, has been described in unicellular organisms including protozoan parasites. Limited information is available regarding programmed cell death in $T$. gondii. However, knowledge of how this parasite dies and whether this proceeds via distinct cellular pathways is a major of interest. Therefore, the focus of this study was to unravel cell death modalities in $T$. gondii and to characterise underlying mechanisms. Specifically, the aims of this study were to:

1. characterise cell death phenotypes in $T$. gondii,

2. determine the physiologic relevance of apoptosis-like cell death in $T$. gondii,

3. identify molecules that may be involved in the regulation of apoptosis-like cell death in T. gondii,

4. examine how cell death in $T$. gondii is regulated.

Since apoptosis is the most common phenotype of cell death, this study focused on the identification of markers of apoptosis in $T$. gondii. We further determined when the parasite employs such form of cell death during its natural life cycle including tachyzoites and bradyzoites that contribute to acute and chronic infection, respectively. In addition, we determined whether the parasite undergoes apoptosis following treatment with proapoptotic stimuli or drug treatment in vitro. We performed in silico analysis of the $T$. gondii genomic database and identified proteins that may play roles in apoptosis mechanism in $T$. gondii. Since caspases or members of the Bcl-2 family have not been identified in the genome of protozoan parasites, programmed cell death, if present, would 
proceed independently of caspases. Endonuclease G and Bax inhibitors are of our interest in this study as both proteins have been reported to play a role in caspase-independent apoptosis pathway. Endonuclease $\mathrm{G}$ is a mitochondria-located endonuclease that is translocated to the nucleus where it cleaves chromatin DNA into nucleosomal fragments. Bax inhibitors are at least partially ER membrane-resident proteins that act as negative regulators of cell death caused by ER stress. Up to now, there are no reports on either $T$. gondii Bax inhibitors or EndoG. Considering the role of these proteins in apoptosis pathways, structure and function of these proteins in the metabolism of T. gondii and particularly in the regulation of apoptosis-like cell death in the parasite needs to be elucidated. We, therefore, in this study tried to identify the presence of genes encoding putative Bax inhibitors and EndoG via analysis from the Toxoplasma genomic database. We further intended to analyse their structure and designed approaches to analyse their function. Moreover, the involvement of mitochondria and proteases during PCD in $T$. gondii was analysed by detecting the mitochondrial membrane potential as well as caspaselike activity in parasites that have been treated with pro-apoptotic stimuli.

Currently available drug regimens against $T$. gondii are only effective against the tachyzoite stage but not the bradyzoite stage. Furthermore, those drugs are poorly tolerated and have severe side effects (Blader and Saeij, 2009). Therefore, the development of new approaches to treat the disease is a challenge to be solved. A better understanding of programmed cell death in $T$. gondii is required. Once the knowledge of molecular mechanisms of PCD in Toxoplasma is known, it would possibly open new possibilities to combat the parasite without strong stimulation of the host immune response. 


\section{CHAPTER 2 \\ MATERIALS AND METHODS}

\subsection{Materials}

Disposable materials and plastic ware used in this study were purchased from Nunc, Falcon, Corning Costar or Braun.

\subsubsection{Cell lines, $T$. gondii and bacteria strains}

All cell lines, Toxoplasma gondii and bacteria, unless otherwise stated, were obtained from stocks available at the Institute for Medical Microbiology, Georg-August University Göttingen.

HFF

HeLa229

L929

ME49 (tissue cysts)

NTE

E. coli $\mathrm{DH} 5 \alpha$

E. coli BL21 (DE)3

E. coli BL21 (DE)3 codon plus
Human foreskin fibroblasts

Human cervical cancer cell line (European Collection of Animal Cell Cultures/ECACC)

Mouse-derived fibroblasts

T. gondii type II strain (kindly provided by Dirk Schlüter, Magdeburg)

T. gondii type II strain (clinical isolate; Groß, 1991)

\subsubsection{Cell death inducers}

All cell death inducers were purchased from Sigma-Aldrich.

Table 2.1. Cell death inducers and concentrations used in this study

\begin{tabular}{lll}
\hline Chemical & Stock solution & Working concentration \\
\hline Brefeldin A & $10 \mathrm{mM}$ in DMSO & $10 \mu \mathrm{M}$ \\
Camptothecin & $50 \mathrm{mg} / \mathrm{ml}$ in DMSO & $1 \mu \mathrm{M}$ and $5 \mu \mathrm{M}$ \\
$\mathrm{H}_{2} \mathrm{O}_{2}$ & $200 \mathrm{mM}$ in ddH $\mathrm{de}_{2} \mathrm{O}$ & $0.5 \mathrm{mM}, 2 \mathrm{mM}$ and $5 \mathrm{mM}$ \\
Miltefosine & $500 \mathrm{mM}$ in PBS & $20 \mu \mathrm{M}$ and $100 \mu \mathrm{M}$
\end{tabular}


Staurosporine

Thapsigargin

Tunicamycin
$1 \mathrm{mM}$ in DMSO

$2 \mathrm{mM}$ in DMSO

$10 \mathrm{mM}$ in DMSO
$0.3 \mu \mathrm{M}, 5 \mu \mathrm{M}$, and $10 \mu \mathrm{M}$

$2 \mu \mathrm{M}$

$5 \mu \mathrm{g} / \mathrm{ml}$

\subsubsection{Antibiotics and parasiticidal agents}

Drugs used in this study, unless otherwise stated, were purchased from Sigma-Aldrich.

Table 2.2. Antibiotics and antiparasite drugs and concentrations used in this study

\begin{tabular}{lll}
\hline Chemical & Stock solution & Working concentration \\
\hline Ampicillin & $100 \mathrm{mg} / \mathrm{ml}$ in $\mathrm{ddH}_{2} \mathrm{O}$ & $100 \mu \mathrm{g} / \mathrm{ml}$ \\
Anhydrotetracycline & $2 \mathrm{mg} / \mathrm{ml}$ in DMF & $25 \mathrm{ng} / \mathrm{ml}$ \\
Anisomycin & $10 \mathrm{mM}$ in $70 \%$ ethanol & $100 \mathrm{nM}$ and $500 \mathrm{nM}$ \\
Atovaquone & $5 \mathrm{mM}$ in DMSO & $100 \mathrm{nM}$ and $1 \mu \mathrm{M}$ \\
Chlorampenicol & $34 \mathrm{mg} / \mathrm{ml}$ in ethanol & $34 \mu \mathrm{g} / \mathrm{ml}$ \\
Clindamycin hydrochloride & $10 \mathrm{mM}$ in $70 \%$ ethanol & $100 \mathrm{nM}$ and $500 \mathrm{nM}$ \\
G418 (Biochrom AG) & $100 \mathrm{mg} / \mathrm{ml}$ in ddH $\mathrm{H}_{2} \mathrm{O}$ & $700 \mu \mathrm{g} / \mathrm{ml}$ \\
Penicillin/Streptomycin & $10,000 \mathrm{U} / \mathrm{ml} / 10,000 \mu \mathrm{g} / \mathrm{ml}$ & $1 \%(\mathrm{v} / \mathrm{v})$ \\
ready to use (Biochrom & & \\
AG) & & $1 \mu \mathrm{M}$ and $5 \mu \mathrm{M}$ \\
Pyrimethamine & $10 \mathrm{mM}$ in $70 \%$ ethanol & \\
\hline
\end{tabular}

\subsubsection{Protease substrate and protease inhibitors}

Table 2.3. Protease substrate and protease inhibitors and concentrations used in this study

\begin{tabular}{lll}
\hline Chemical & Stock solution & Working concentration \\
\hline DEVD-AMC (Bachem) & $10 \mathrm{mM}$ in $\mathrm{ddH}_{2} \mathrm{O}$ & $10 \mu \mathrm{M}$ \\
E64 (Sigma-Aldrich) & $20 \mathrm{mg} / \mathrm{ml}$ in DMSO & $100 \mu \mathrm{M}$ \\
Protease inhibitor cocktail & 1 tablet dissolved in $400 \mu \mathrm{l}$ & $4 \%(\mathrm{v} / \mathrm{v})$ \\
(Roche) & $\mathrm{ddH}_{2} \mathrm{O}$ & \\
Z-VAD-FMK (Enzo Life & $10 \mathrm{mg} / \mathrm{ml}$ in DMSO & $100 \mu \mathrm{M}$ \\
Sciences/Alexis) & & \\
\hline
\end{tabular}




\subsubsection{Antibodies}

Table 2.4. Antibodies used for Western blot (WB) or immunofluorescence assay (IFA) and concentrations used in this study

\begin{tabular}{|c|c|}
\hline Antibodies & $\begin{array}{l}\text { Working dilution (WB; } \\
\text { IFA) }\end{array}$ \\
\hline Anti-FLAG M2 (Stratagene) & IFA $1: 800$ \\
\hline Anti-His-tag Ab (Qiagen) & WB $1: 1,000$ \\
\hline Anti-mouse-IgG HRP conjugate (Dianova) & WB 1:2,000 \\
\hline Cy2-conjugated anti-mouse IgG (Dianova) & IFA $1: 400$ \\
\hline Cy3-conjugated donkey anti-rabbit IgG (Dianova) & IFA $1: 800$ \\
\hline Cy3-conjugated anti-rat antibody IgG (Dianova) & IFA $1: 400$ \\
\hline $\begin{array}{l}\text { DC11 (rat anti-bradyzoites antibody; Institute for Medical } \\
\text { Microbiology, Georg-August University Göttingen) }\end{array}$ & IFA $1: 100$ \\
\hline QIAexpress Strep-tag Ab (Qiagen) & WB $1: 2,000$ \\
\hline $\begin{array}{l}\text { RS7.2 (rabbit anti-T. gondii polyclonal antibody; Institute } \\
\text { for Medical Microbiology, Georg-August University }\end{array}$ & IFA $1: 1,000$ \\
\hline Göttingen) & \\
\hline
\end{tabular}

\subsubsection{Fluorescent probes}

In this study, Hoechst 33258 and propidium iodide (both were from Sigma-Aldrich) were used to stain nucleic acids. To label mitochondria with an intact mitochondrial membrane potential, MitoTracker ${ }^{\circledR}$ Orange CM-H ${ }_{2}$ TMRos (Invitrogen) was applied.

\subsubsection{Oligonucleotides}

Primer sequences used in this study were designed using Primer3 software and were purchased from Sigma-Aldrich. 
Table 2.5. Primer pairs for amplifying cell death-related genes via quantitative real time PCR

\begin{tabular}{|c|c|c|c|}
\hline Gene ID & Forward $\left(5^{\prime}-3^{\prime}\right)$ & Reverse $\left(5^{\prime}-3^{\prime}\right)$ & $\begin{array}{l}\text { Amplicon } \\
\text { (bp) }\end{array}$ \\
\hline TgME49_008710 & AGGCAGCGAAACACAGAAAG & ACATGCAGGGAAGCTCCA & 264 \\
\hline TgME49_007620 & CGCGTACCCATACGTGAAGA & GCGACGAACTGTTGCTTGTC & 234 \\
\hline TgME49_078970 & ACTTCGTCGCGTTTCACTTT & ATGCAGTACGCATTGAGCAG & 248 \\
\hline TgME49_053000 & AAGGCGAGAGGAATGGATTT & CGTCGTAAAATTGCAGCAGA & 201 \\
\hline TgME49_094420 & AGAGAGGAGGGAGACGAAGG & CAGCGAACTGTGTTCGTCAT & 156 \\
\hline TgME49_093820 & GACGATTCACACATGGTTGC & TTGATTTTCCTGGGAGAACG & 228 \\
\hline TgME49_093830 & GACCTCTCGGCTGTAGCATC & CGCACCGTCTGTCAGAGTTA & 158 \\
\hline TgME49_021360 & CTTCGTCAGCGGGAATATGT & GCCGATAGTGAGTTGGGTGT & 241 \\
\hline TgME49_049770 & GCCATTGTTCCACGTCTTTT & GAAGGGGAAGGAAGGAGATG & 162 \\
\hline TgME49_109560 & CCAGGACAATTTGCACACAC & CGTCCGCTCTTTTCTTTACG & 232 \\
\hline TgME49_003030 & CCAGGCTGCTACTCCTTACG & GAACCCGTTTCAATGTCGAT & 171 \\
\hline TgME49_105490 & TCTTGCCAGCGTGAATACTG & GCGCTGATAGTCACTCCACA & 247 \\
\hline TgME49_105870 & GATGCATTCTGTGCGTGTCT & GGGACTCACCTGGATTCTGA & 159 \\
\hline TgME49_035560 & TCCCAGTCCACAGAGTTCCT & AGAGACGGCAAAGGATCAGA & 209 \\
\hline TgME49_107780 & GTTGCTGGGATAGCTTCTGC & GCCTTGAGAGGACAGAGGTG & 240 \\
\hline TgME49_035880 & CCGAGCGAAGTTTTTACGTC & CACCGAAAATGTGAGACACG & 170 \\
\hline TgME49_006490 & TTCGTCCACTACGCTTGTTG & GGGAGAATCGTTTCGTCGTA & 207 \\
\hline $\begin{array}{l}\text { TgME49_009030 } \\
\text { (actin) }\end{array}$ & TGGCAACGAGCGATTCCGCTG & GTTCCTTGGTCAGCCTCTCGCC & 200 \\
\hline
\end{tabular}

Table 2.6. Primer pairs for cloning and sequencing of $T$. gondii Endonuclease G

\begin{tabular}{ll}
\hline Primers & Sequence $\left(5^{\prime}-3^{\prime}\right)$ \\
\hline TgEG_KlonipQE30_2_for & NNNNNNGCATGCACTACTCCTTTCGTTTCTCCTCCTTC \\
& $($ SpHI site in bold letters $)$ \\
TgEG_KlonipQE30_2_rev & NNNNNNAAGCTTTCAGTCGCCCAGAACAGAAGCG \\
& (HindIII site in bold letters $)$ \\
TgEG_KlonipASKIBA3_2_for & NNNNNNGAATTCACTACTCCTTTCGTTTCTCCTCCTTC \\
& TGC (EcoRI site in bold letters $)$
\end{tabular}




$\begin{array}{ll}\text { TgEG_KlonipASKIBA3_2_rev } & \begin{array}{l}\text { NNNNNNGGTACCGTCGCCCAGAACAGAAGCGATG } \\ (\text { KpnI site in bold letters })\end{array} \\ \text { TgEndoG_4_for } & \text { ACGCCACTGTCCAGCCTTCG } \\ \text { TgEndoG_4_rev } & \text { CCGCCAGATGCCCCTTGCTG } \\ \text { TgEndoG893_1486_for } & \text { CCGAAAGGCTCTGTTGTTTC } \\ \text { TgEndoG893_1486_rev } & \text { TTTTTCGCCTCTTTCGTCTC } \\ \text { TgEndoG1364_2175_for } & \text { CTTTCAGGCAAGGGATTCTC } \\ \text { TgEndoG1364_2175_rev } & \text { TCGAGCCTGACTCTCTCCTC } \\ \text { TgEndoG_1911-1931 } & \text { AAACCCTCAGACGCCTTCTT } \\ \text { TgEndoG_2380-2400 } & \text { GTCGTAGTGGAGGCCATTGT }\end{array}$

\subsubsection{Plasmids}

TgEndoG-pBluescriptSK- Recombinant plasmid containing TgEndoG full length sequence (purchased from ATG:biosynthetics GmBH). pASK-IBA3 plus Expression plasmid. It contains an anhydrotetracycline (ATc)-regulatable promoter for generating C-terminal Strep-tagged proteins (IBA GmbH).

pQE30 For recombinant protein expression in E. coli-generating proteins fused with a $6 x$ His-tag at the $\mathrm{N}$-terminus (Stratagene).

pRSET-A For recombinant protein expression in E. coli-generating proteins fused with a 6xHis-tag at the N-terminus (kindly provided by Antonio Jiménez-Ruiz, Madrid).

\subsubsection{Molecular weight markers}

GeneRuler 100 bp DNA Ladder

Fermentas

GeneRuler 1 kb DNA Ladder

Fermentas

Prestained Protein Marker, Broad Range

(6-175 kDa)

New England Biolabs 


\subsubsection{Enzymes}

Alkaline antarctic phosphatase

DNase I Amplification Grade

KOD DNA polymerase

Omniscript reverse transcriptase

Quick T4 DNA Ligase

Restriction Enzymes

RNase A

Taq DNA polymerase
New England Biolabs

Sigma-Aldrich

Novagen

Qiagen

New England Biolabs

New England Biolabs

Sigma-Aldrich

Roche

\subsubsection{Kits}

Annexin V-PE apoptosis detection

BD Biosciences

$\mathrm{BCA}^{\mathrm{TM}}$ Protein Assay

Pierce

CaspaTag ${ }^{\text {TM }}$ Pan-Caspase In Situ Assay Kit, Fluorescein

ECL detection reagent

Chemicon International

FuGene HD Transfection Reagent

Amersham

GenElute $^{\mathrm{TM}}$ Mammalian Total RNA Miniprep

Roche

GenElute Plasmid Mini Prep Kit

Sigma-Aldrich

GenElute Plasmid Maxi Prep Kit

Sigma-Aldrich

In Situ Cell Death Detection, Fluorescein

Sigma-Aldrich

LightCycler ${ }^{\circledR}$ FastStart DNA Master ${ }^{\text {PLUS }}$ SYBR Green I

Roche

Omniscript ${ }^{\circledR}$ Reverse Transcription

Roche

QIAquick PCR Purification Kit

Qiagen

Qiagen

Quick Ligation Kit

New England Biolabs

\subsubsection{Other chemicals and reagents}

Acrylamide/bisacrylamide

Roth

Agarose NEEO Ultra-Qualität

Roth

Ammonium peroxodisulfate (APS)

Sigma-Aldrich

Ampuwa sterile water

Sartorius

BSA

Sigma-Aldrich 


\section{CHAPS}

Diethanolamine

Dimethylformamid

EDTA

Mowiol

PBS

Ponceau S

Roti ${ }^{\circledR}$-Blue

Saponin

Sodium azide

Sodium citrate

TEMED

Triton X-100

Trypsin

Tween 20

\subsubsection{Instruments}

Centrifuges

Digital balances

Electrophoresis power supply

FACS

Gel documentation system

Gel electrophoresis

Incubators
Sigma-Aldrich

Fluka BioChemika

Fluka BioChemika

Merck

Calbiochem

Biochrom AG

Roth

Roth

Sigma-Aldrich

Merck

Biochrom AG

Roth

Sigma-Aldrich

Biochrom AG

Biochrom AG

Megafuge 2.0 (Heraeus)

Megafuge 2.0 RS (Heraeus)

Multifuge 3 S-R (Heraeus)

Avanti J-25 (Beckman Coulter)

5417 R (Eppendorf)

5424 (Eppendorf)

5403 (Eppendorf)

LP6200 S (Sartorius)

CP 224 S (Sartorius)

EPS 500/400 (Pharmacia Fine Chemicals)

FACSCalibur (BD Biosciences)

BioDoc II (Biometra)

Mini Protean II (BioRad)

Type BB 6220 CU (Heraeus) 
Laser scanning confocal microscope

Light cycler

Light microscopy

Luminescent image analyzer

Luminometry

Magnetic stirrer

$\mathrm{pH}$ Meter

Photometer

Pipette aids

Pipettes

Semi-dry gel transfer

Sonicator

Shaker

Thermocycler

Thermomixer
TCS SP 2 (Leica)

LightCycler ${ }^{\circledR}$ Carousel-Based System (Roche)

Model DM IL (Leica)

Axiostar plus (Zeiss)

Image Reader LAS-4000 (Fujifilm)

Victor V multilabels counter (Perkin Elmer)

RCT basic (IKA Labortechnik)

Calimatic 766 (Knick)

Ultraspec 1000 (Pharmacia Biotech)

Pipetboy acu (IBS Integra Biosciences)

Pipetus-akku (Hirschman Laborgerate)

Eppendorf

Semi-dry electroblotter (Sartorius)

Sonifier 250 (Branson)

Polymax 1040 (Heidolph)

Duomax 1030 (Heidolph)

Model T3 (Biometra)

Model Compact (Eppendorf)

\subsection{Methods}

\subsubsection{Maintenance of cells and cell harvest}

\subsubsection{In vitro cultivation of Toxoplasma gondii}

Toxoplasma gondii strain NTE was used for all experiments involving T. gondii tachyzoites. Tachyzoites were propagated in L929 cells in 12 -well plates at $37^{\circ} \mathrm{C}$, in a $5 \%$ $\mathrm{CO}_{2}$ humidified atmosphere in Toxoplasma medium (see below). Extracellular parasites released from lysed host cells (approximately 3-4 days post inoculation/p.i) were used for re-inoculation at different parasite-host cell ratios, ranging from 6 to 2 drops of parasites and 5 to 3 drops of L929 in $1.5 \mathrm{ml}$ Toxoplasma medium. 


\section{$\underline{\text { Toxoplasma medium }}$}

RPMI 1640

$1 \%(\mathrm{v} / \mathrm{v})$ heat-inactivated FCS

$100 \mathrm{U} / \mathrm{ml}$ Penicillin/100 $\mu \mathrm{g} / \mathrm{ml}$ Streptomycin

\subsubsection{Production of brain cysts}

The avirulent type II ME49 cysts-forming strain of $T$. gondii was a kind gift from Dirk Schlüter, Magdeburg. Brains from chronically infected mice were isolated at 10 to 30 weeks p.i, homogenised using glass beads and spread onto glass slides for further experiments. To infect mice for brain cyst development, 5-10 cysts were intraperitoneally inoculated into NMRI mice.

\subsubsection{Cultivation of wild type and transfected cell lines}

All cell lines were grown at $37^{\circ} \mathrm{C}, 5 \% \mathrm{CO}_{2}$ in a humidified atmosphere. Human Foreskin Fibroblast (HFF), HeLa cells and Toxoplasma gondii Bax inhibitors (TgBIs)-expressing HeLa cells were grown in $75 \mathrm{~cm}^{2}$ flasks with DMEM medium supplemented as indicated below. The cells were detached by rinsing with 3-5 $\mathrm{ml} 1 \%$ (w/v) EDTA (Biochrom AG) and subsequent incubation in 3-5 ml $0.25 \%(\mathrm{w} / \mathrm{v})$ trypsin (Biochrom AG). After resuspension in cell culture medium, the cells were then centrifuged at $400 \mathrm{x} \mathrm{g}$ for $5 \mathrm{~min}$ and used for subcultivation. Subcultivation of L929 was done by scraping the cells using a cell scraper, and 6 to 8 drops of cell suspension were transferred into a 6-well plate with 4 ml L929 medium. Subcultivation was done twice a week.

\section{L929 medium}

DMEM

$1 \%(\mathrm{v} / \mathrm{v})$ heat-inactivated FCS

100 U/ml Penicillin/

$100 \mu \mathrm{g} / \mathrm{ml}$ Streptomycin

$1 \mathrm{x}$ non-essential amino acid

$1 \mathrm{mM}$ sodium pyruvate
$\underline{\mathrm{HeLa} / \mathrm{HFF} \text { medium }}$

DMEM

$10 \%(\mathrm{v} / \mathrm{v})$ heat-inactivated FCS

100 U/ml Penicillin/

$100 \mu \mathrm{g} / \mathrm{ml}$ Streptomycin

$1 \mathrm{x}$ non-essential amino acid

$1 \mathrm{mM}$ sodium pyruvate 


\subsubsection{Harvesting of $T$. gondii tachyzoites}

Extracellular tachyzoites were harvested by differential centrifugation at $35 \mathrm{x} \mathrm{g}$ for $5 \mathrm{~min}$ to separate them from host cells. The supernatant, which contained the parasites, was then transferred into a fresh tube and centrifuged at 1,314 x g for $10 \mathrm{~min}$. Pelleted parasites were resuspended in $10 \mathrm{ml}$ Toxoplasma medium and centrifuged again at 1,314 x g for 10 min. The pellet was then resuspended in $1 \mathrm{ml}$ medium and parasites were counted in a hemocytometer grid (see below).

\subsubsection{Harvesting mammalian cells}

HFF, HeLa, or TgBIs-transfected HeLa cells were detached using EDTA and trypsin as described above (section 2.2.1.3). After centrifugation, cells were washed once by resuspension in cell culture medium and centrifugation at $400 \mathrm{x}$ g for $5 \mathrm{~min}$. Pelleted cells were resuspended in $\mathrm{HeLa} / \mathrm{HFF}$ medium. An appropriate dilution of the cells was then mixed with an equal volume of $0.1 \%$ (w/v) tryphan blue in PBS in order to determine the number of viable cells using a hemocytometer grid.

\subsubsection{Cell count}

A hemocytometer (Improved Neubauer) was used to determine the amount of cells by using the formula:

Number of cells per $\mathrm{ml}=$ average counted cells per quadrant $\mathrm{x}$ dilution factor $\mathrm{x} 10^{4}$.

\subsubsection{Cryopreservation of cells}

After counting the cells, $1 \times 10^{7}$ cells per ml were mixed gently with an equal volume of freezing medium (see below) and transferred in a cryotube. The cells were stored immediately at $-80^{\circ} \mathrm{C}$ overnight before transferring them into liquid nitrogen for long-term storage. To recultivate frozen cells, they were thawed in a $37^{\circ} \mathrm{C}$ waterbath and were immediately transferred into a 50-ml centrifuge tube with the appropriate cell culture medium. After centrifugation at $400 \mathrm{x} \mathrm{g}$ for $5 \mathrm{~min}$ (mammalian cells) or $1,314 \mathrm{x} \mathrm{g}$ for 10 min (T. gondii), pelleted cells were resuspended in cell culture medium, transferred into a flask or co-cultivated with host cells, and incubated at $37^{\circ} \mathrm{C}$ in a $5 \% \mathrm{CO}_{2}$ humidified atmosphere. 


\section{Freezing medium}

$40 \%$ cell culture medium

$40 \%(\mathrm{v} / \mathrm{v}) \mathrm{FCS}$

$20 \%(\mathrm{v} / \mathrm{v}) \mathrm{DMSO}$

\subsubsection{Determination of apoptosis markers}

\subsubsection{DNA strand breaks and cell size reduction in extracellular parasites}

Terminal deoxynucleotidyl transferase-mediated X-dUTP nick end labelling (TUNEL) assay followed by flow cytometry was employed to determine DNA strand breaks and reduction of cell volume, which are hallmarks of apoptosis. For this purpose, tachyzoites were seeded at a cell density of $2 \times 10^{7}$ parasites in $500 \mu 1$ Toxoplasma medium in a 24well plate for $72 \mathrm{~h}$. The parasites were treated with $5 \mu \mathrm{M}$ and $10 \mu \mathrm{M}$ staurosporine, $20 \mu \mathrm{M}$ and $100 \mu \mathrm{M}$ miltefosine, $0.5 \mathrm{mM}$ and $2 \mathrm{mM} \mathrm{H}_{2} \mathrm{O}_{2}, 1 \mu \mathrm{M}$ and $5 \mu \mathrm{M}$ camptothecin, or were left untreated. They were harvested at 0 (freshly isolated parasites), 6, 24, 48, and $72 \mathrm{~h}$ post treatment. All samples were centrifuged at $1,800 \mathrm{x}$ g for $10 \mathrm{~min}$, washed with PBS and fixed in $4 \%(\mathrm{w} / \mathrm{v})$ paraformaldehyde in $0.1 \mathrm{M}$ sodium cacodylate $\mathrm{pH} 7.4$ for $1 \mathrm{~h}$ at room temperature. TUNEL staining was performed using the In Situ Cell Death Detection, Fluorescein kit (Roche) as recommended in the manufacturer's instruction. Briefly, after washing with PBS, the fixed cells were irreversibly permeabilised with $0.1 \%$ Triton X-100 in $0.1 \%$ sodium citrate for $5 \mathrm{~min}$ at room temperature. The cells were then centrifuged at $1,800 \mathrm{x}$ g for $10 \mathrm{~min}$. Pelleted cells were resuspended in $30 \mu \mathrm{l}$ TUNEL solution (1:10 of enzyme in labelling solution), and incubated for $1 \mathrm{~h}$ at $37^{\circ} \mathrm{C}$ in the dark. The cells were then washed once with PBS. Finally, cells were resuspended in $500 \mu 1$ PBS and measured by flow cytometry using a FACSCalibur (BD Bioscience) and analysed by Cellquest Pro as software. A total of 50,000 events were collected from each sample. TUNEL-positive cells were determined from the logarithmic FL-1 Height histogram. An alteration in cell size was measured in parallel by comparing the mean of forward scatter (FSC) value from different samples.

To evaluate the TUNEL reactivity of $T$. gondii microscopically, a $50 \mu 1$ aliquot of parasite suspension treated or not with pro-apoptotic stimuli was spread onto glass slides, air-dried, and fixed in $4 \%(\mathrm{w} / \mathrm{v})$ paraformaldehyde in $0.1 \mathrm{M}$ sodium cacodylate $\mathrm{pH} 7.4$ for $1 \mathrm{~h}$ at 
room temperature. The samples were blocked/permeabilised in $0.1 \mathrm{mg} / \mathrm{ml}$ saponin and $1 \%$ BSA in PBS (blocking solution) for $1 \mathrm{~h}$ at room temperature. After having been washed with $0.1 \mathrm{mg} / \mathrm{ml}$ saponin in PBS (washing solution) for $10 \mathrm{~min}$, the cells were incubated with rabbit anti-T. gondii polyclonal antibody RS7.2 (diluted 1:1,000 in blocking solution) for $1 \mathrm{~h}$ at room temperature. After three washing steps (10 min for each step) with washing solution, the cells were incubated with Cy3-conjugated donkey anti-rabbit IgG (diluted 1:800 in blocking solution). Following three washing steps, the cells were irreversibly permeabilised with $0.1 \%$ Triton $\mathrm{X}-100$ in $0.1 \%$ sodium citrate for at least $5 \mathrm{~min}$ at room temperature. The parasites were then incubated in $60 \mu 1$ TUNEL mixture to ensure proper distribution of the solution on the parasites. Afterwards, the cells were washed twice with PBS and mounted using Mowiol 4-88 (Calbiochem). The number of TUNEL-positive cells was counted from at least 350 parasites using confocal laser scanning microscopy with a $63 x$ oil-immersion objective. In some experiments, nuclei were counterstained with Hoechst 33258. To this end, after TUNEL staining, the parasites were washed twice in PBS and were then incubated in $2 \mu \mathrm{g} / \mathrm{ml}$ Hoechst 33258 (stock $1 \mathrm{mg} / \mathrm{ml}$, diluted in 0.1 $\mathrm{mg} / \mathrm{ml}$ saponin in PBS) for $45 \mathrm{~min}$ at room temperature in the dark. Afterwards, cells were washed three times with PBS, rinsed shortly in $\mathrm{ddH}_{2} \mathrm{O}$ and mounted using Mowiol 4-88. The nuclei stained with TUNEL and Hoechst 33258 were documented using a conventional fluorescence microscopy (Zeiss).

\subsubsection{DNA strand breaks in intracellular parasites}

To examine the presence of TUNEL-positive intracellular parasites upon cell death induction, confluent HFF cells grown on glass cover slips in a 24-well cell culture plate were inoculated with $10^{5}$ tachyzoites per well. After $24 \mathrm{~h}$ of infection, $10 \mu \mathrm{M}$ staurosporine or $20 \mu \mathrm{M}$ miltefosine was added. T. gondii-infected non-treated HFF cells were used as controls. The cells were fixed after 3,6 , and $24 \mathrm{~h}$ post treatment with $4 \%$ paraformaldehyde in $0.1 \mathrm{M}$ sodium cacodylate $\mathrm{pH} 7.4$ for $1 \mathrm{~h}$ at room temperature. Samples were simultaneously blocked/permeabilised in $0.1 \mathrm{mg} / \mathrm{ml}$ saponin and $1 \%$ BSA in PBS for $1 \mathrm{~h}$ at room temperature. The parasites were then labelled with parasite-specific antibody and TUNEL as described above (section 2.2.2.1). Cells were examined by confocal laser scanning microscopy. 


\subsubsection{Detection of phosphatidylserine exposure}

An Annexin V-Phycoerythrin (PE) apoptosis detection kit was used to quantify the translocation of phosphatidylserine from the inner to the outer leaflet of the plasma membrane after treatment of parasites with pro-apoptotic stimuli. To this end, $2 \times 10^{7}$ parasites were incubated in the presence of $10 \mu \mathrm{M}$ staurosporine or $20 \mu \mathrm{M}$ miltefosine for 24 and 48 h. As controls, non-treated parasites were used. Samples were washed twice with ice-cold PBS and then resuspended in $1 \mathrm{x}$ Annexin V-binding buffer (provided in the kit) at a concentration of $1 \times 10^{6}$ cells per ml. A $100 \mu$ aliquot of parasite suspension $(1 \mathrm{x}$ $10^{5}$ cells) was then mixed with $5 \mu \mathrm{l}$ of Annexin V-solution and $5 \mu \mathrm{l}$ of 7-AAD-solution, and was incubated for $15 \mathrm{~min}$ at room temperature in the dark. After adding $400 \mu \mathrm{l}$ of $1 \mathrm{x}$ Annexin V-binding buffer to each sample, cells were analysed by flow cytometry. Nonstained cells, cells stained with Annexin V alone, and cells stained with 7-AAD alone were used as controls to set up compensation parameters and quadrants. Data from 10,000 events per sample was collected using a BD FACSCalibur flow cytometer and analysed using Cellquest Pro as software.

\subsubsection{Effect of pro-apoptotic stimuli on parasite invasiveness}

To evaluate the ability of extracellular parasites that have been treated with pro-apoptotic stimuli to invade host cells, $2 \times 10^{7}$ parasites were pre-treated with $10 \mu \mathrm{M}$ staurosporine or $20 \mu \mathrm{M}$ miltefosine or were left untreated for $48 \mathrm{~h}$. At the indicated time point, parasites were isolated and washed once with HFF cell culture medium by centrifugation at 1,800 $\mathrm{x}$ $\mathrm{g}$ for $10 \mathrm{~min}$. Confluent HFF cells which have been seeded on glass cover slips within the wells of a 24 -well plate were then inoculated with $2 \times 10^{5}$ parasites per well in the absence of pro-apoptotic stimuli. After $4 \mathrm{~h}$, the medium was exchanged to remove extracellular parasites and cells were incubated for further $48 \mathrm{~h}$. Cells were then fixed in $4 \%(\mathrm{w} / \mathrm{v})$ paraformaldehyde in $0.1 \mathrm{M}$ sodium cacodylate $\mathrm{pH} 7.4$ for $1 \mathrm{~h}$ at room temperature and parasites were stained with parasite-specific antibody as described above (section 2.2.2.1, without TUNEL staining). The number of parasites which had invaded host cells was counted in 10 randomly selected visual fields per sample. Results were presented as the number of parasitophorous vacuoles per visual field. 


\subsubsection{Physiological significance of apoptosis-like cell death in $T$. gondii}

\subsubsection{TUNEL staining of $T$. gondii from the peritoneal cavity during acute toxoplasmosis in mice}

NMRI mice were inoculated with 50, 100 or 500 T. gondii NTE tachyzoites i.p. At 7, 10 and 12 days p.i, the mice were killed by $\mathrm{CO}_{2}$ inhalation and the intraperitoneal fluid was collected by first injecting 7-8 $\mathrm{ml}$ ice-cold $\mathrm{PBS}$ and then aspirating the diluted intraperitoneal exudate. After centrifugation at $35 \mathrm{x} \mathrm{g}$ for $5 \mathrm{~min}$, the supernatant was transferred into a new tube and was centrifuged again at 1,314 x g for $10 \mathrm{~min}$. Pelleted cells that contain parasites was resuspended in $1 \mathrm{ml} 1 \mathrm{x}$ PBS. An aliquot of $50 \mu \mathrm{l}$ intraperitoneal fluid was then spread onto slides, air-dried, and fixed in $4 \%(\mathrm{w} / \mathrm{v})$ paraformaldehyde in $0.1 \mathrm{M}$ sodium cacodylate $\mathrm{pH} 7.4$ for $1 \mathrm{~h}$ at room temperature. Finally, parasites were stained as described above (section 2.2.2.1).

\subsubsection{TUNEL staining of brain cysts from chronically infected mice}

Homogenised brain from chronically Toxoplasma-infected mice (see 2.2.1.2) was spread on glass slides, air-dried and fixed in ice-cold absolute methanol at room temperature for 5 min. The cells were then blocked/permeabilised in $1 \%$ BSA and $0.2 \%$ Triton X-100 in PBS pH 7.4 (blocking solution) for $1 \mathrm{~h}$. Thereafter, bradyzoites-containing tissue cysts were immunolabelled with DC11 rat anti-T. gondii bradyzoite antibody (diluted at 1:100 in blocking solution) for $1 \mathrm{~h}$. After having been washed three times in PBS, cells were incubated in Cy3-conjugated anti-rat IgG antibody (diluted 1:400 in blocking solution) for $1 \mathrm{~h}$ in the dark. Afterwards, cells were washed as above and were incubated in $0.1 \%$ Triton X-100 in $0.1 \%$ sodium citrate for $15 \mathrm{~min}$ and then TUNEL-stained as described in section 2.2.2.1. Samples were examined by confocal laser scanning microscopy.

\subsubsection{Apoptosis-like cell death after treatment of parasites with chemotherapeutic agents}

HFF cells were seeded in 24-well plates containing $13 \mathrm{~mm}$ round cover slips at a density of 40,000 cells per well. After having reached confluence, the cells were infected with $10^{5}$ tachyzoites per well. Four hours after infection, the monolayer cells were washed to remove extracellular parasites and cell culture medium was replaced by fresh medium 
containing $100 \mathrm{nM}$ and $500 \mathrm{nM}$ anisomycin, $1 \mu \mathrm{M}$ and $5 \mu \mathrm{M}$ pyrimethamine, $100 \mathrm{nM}$ and $1 \mu \mathrm{M}$ atovaquone or $100 \mathrm{nM}$ and $500 \mathrm{nM}$ clindamycin. Infected cells cultured without any drugs were used as controls. At 24 and $48 \mathrm{~h}$ post treatment, cells were fixed in $4 \%(\mathrm{w} / \mathrm{v})$ paraformaldehyde in $0.1 \mathrm{M}$ sodium cacodylate $\mathrm{pH} 7.4$ for $1 \mathrm{~h}$ at room temperature. Due to the delayed anti-parasitic effect of clindamycin (Fichera et al., 1995; Pfefferkorn et al., 1992), treatment with this drug was done during two consecutive cycles. Samples of the first cycle were simultaneously fixed with other treated samples. For the second cycle of treatment, parasites which egressed from the host cells during the first cycle of clindamycin treatment (3-4 days p.i) were isolated by centrifugation at 1,800 x g for 10 min. Pelleted parasites were resuspended in HFF cell culture medium and fresh HFF cells were infected with $25 \%$ of parasites from the first cycle in the presence of clindamycin. At 24 and $48 \mathrm{~h}$ after the addition of drugs, staining of parasites and TUNEL test were performed as described above. Parasite replication was determined by counting the number of tachyzoites per parasitophorous vacuole (at least 100 parasitophorous vacuoles per sample were inspected). The presence of TUNEL-positive parasites was monitored and documented by confocal laser scanning microscopy with a 63x oil-immersion objective.

\subsubsection{Putative regulators of apoptosis-like cell death in $T$. gondii}

To identify putative cell death regulators of apoptosis-like cell death in T. gondii, in silico analysis of the Toxoplasma genomic database (www.toxodb.org) was performed. Seventeen genes associated with cell death have been identified using a text-centered approach from the Toxoplasma genomic database (Chapter 3, Table 3.1).

\subsubsection{RNA isolation and cDNA synthesis}

Total RNA was extracted from $7 \times 10^{7}$ parasites, which had been previously incubated with $10 \mu \mathrm{M}$ staurosporine or $20 \mu \mathrm{M}$ miltefosine for 24 and $48 \mathrm{~h}$. RNAs from treated parasites, from freshly isolated parasites and non-treated controls were isolated according to the manufacturer's instruction (GenElute ${ }^{\mathrm{TM}}$ Mammalian Total RNA Miniprep). Contaminating DNA was digested by incubation of $50 \mu$ l of isolated total RNA with $5 \mu 1$ DNase I and $5 \mu$ l DNase buffer (provided in the DNase I Amplification Grade kit, SigmaAldrich) for $15 \mathrm{~min}$ at room temperature. The reaction was stopped by adding $5 \mu$ stop 
solution and DNase was inactivated by incubation for $10 \mathrm{~min}$ at $60^{\circ} \mathrm{C}$. For the cDNA synthesis reaction, up to $2 \mu \mathrm{g}$ DNase-treated RNA were mixed with 1 x RT buffer, $0.5 \mathrm{mM}$ of each dNTP, $10 \mathrm{U}$ RNase inhibitor, $1 \mu \mathrm{M}$ oligodT primer and $4 \mathrm{U}^{\text {Omniscript }}{ }^{\circledR}$ Reverse Transcriptase and RNase-free water in a total volume of $20 \mu 1$. Synthesis of cDNA was carried out for $90 \mathrm{~min}$ at $37^{\circ} \mathrm{C}$.

\subsubsection{Primer design and quantitative real-time PCR}

The synthesized cDNA was subjected to quantitative real time PCR using the primer pairs as shown in Table 2.5 (see 2.1.7). All primer pairs were designed using Primer3 software and were purchased from Sigma-Aldrich. For $20 \mu \mathrm{l} \mathrm{PCR} \mathrm{reactions,} \mathrm{the} \mathrm{following}$ components were mixed: $2 \mu \mathrm{l}$ of cDNA, $0.25 \mu \mathrm{M}$ each of forward and reverse primers, and $4 \mu \mathrm{l}$ of buffer and enzyme mixture (provided in the LightCycler ${ }^{\circledR}$ FastStart DNA Master $^{\text {PLUS }}$ SYBR Green I kit, Roche). The amplification was carried out in a LightCycler ${ }^{\circledR}$ Carousel-Based System (Roche) using a cycling program as follows: (i) denaturation at $95^{\circ} \mathrm{C}$ for $10 \mathrm{~min}, 45$ cycles of (ii) denaturation at $95^{\circ} \mathrm{C}$ for $10 \mathrm{sec}$, (iii) annealing at $62^{\circ} \mathrm{C}$ (with the exception of calpain: $65^{\circ} \mathrm{C}$ ) for $5 \mathrm{sec}$, and (iv) extention at $72^{\circ} \mathrm{C}$ for $20 \mathrm{sec}$, (v) melting curve analysis between $62^{\circ} \mathrm{C}$ and $95^{\circ} \mathrm{C}$, (vi) final incubation at $40^{\circ} \mathrm{C}$ until stopping the PCR. The up- or down-regulation of transcript levels between two samples was calculated on basis of the crossing point $(\mathrm{CP})$ values from the samples which were normalized to actin using the formula:

Level of expression (treatment/freshly) $=\frac{2 \text { target }{ }^{(\text {CPfreshly-CPtreatment })}}{2 \text { actin }{ }^{\text {(CPfreshly-CPtreatment })}}$

Target refers to putative cell death-associated genes.

\subsubsection{Heterologous expression of Toxoplasma gondii Bax inhibitors (TgBIs)}

\subsection{Transfection of TgBIs-coding genes into HeLa cells}

In silico analysis of the Toxoplasma genomic database revealed the presence of three putative T. gondii Bax inhibitors (TgBI-1, TgBI-2, and TgBI-3). Genes encoding TgBIs (identified and cloned in the lab of Dominique Soldati-Favre, Geneva) had previously been subcloned into a FLAG tag-containing mammalian expression vector (pCMV-SC-NF) and recombinant plasmids were extracted using the GenElute Plasmid Maxi Prep Kit (Sigma- 
Aldrich). Subcloning TgBIs coding sequences into pCMV-SC-NF, propagation of the recombinant plasmids and extraction of the plasmids have been done by Julia Hofhuis, a master student who performed her lab rotation at the Institute for Medical Microbiology, Georg-August University of Göttingen.

In order to analyse the function of TgBIs in a well-established system of apoptosis, genes encoding these proteins were heterologously expressed in HeLa cells. To this purpose, HeLa cells were seeded in a 12-well plate at a density of 150,000 cells per well for $24 \mathrm{~h}$ in complete HeLa medium (section 2.2.1.3). The medium was then replaced by DMEM containing $3 \%$ FCS prior to the transfection procedure. Transfection was done using FuGene HD Transfection Reagent (Roche) according to the manufacturer's recommendation (ratio of transfection reagent to recombinant plasmid 4:2 and 6:2) for 15 min at room temperature. The transfection solution was added to the cells in a drop-wise manner. The plates were swirled to ensure gentle mixing of the transfection solution and were then incubated for $4 \mathrm{~h}$ at $37^{\circ} \mathrm{C}$ before changing the medium to complete HeLa cell culture medium again. At $48 \mathrm{~h}$ following transfection, G418 $(700 \mu \mathrm{g} / \mathrm{ml})$ was added for selection of stable transfectants. After several days ( $\sim$ days), the medium was replaced with fresh medium containing G418. After having reached confluence, transfectants were transferred into 6-well plates, and afterwards into $75 \mathrm{~cm}^{2}$ flask. As controls, non-transfected HeLa cells were grown in complete medium without and with G418. Stable transfection of the genes encoding TgBIs into HeLa cells was confirmed by immunofluorescence microscopy (see below).

To obtain single clones of each TgBI-transfected HeLa cell line, pools of stably transfected HeLa-TgBI-1, HeLa-TgBI-2 and HeLa-TgBI-3 were seeded at one cell per well in 96-well plates (in complete HeLa medium supplemented with G418). Cells from single clones per well were selected (approximately at 7 days after seeding) and were transferred into 6-well plates for further propagation. Cells from single clones were then evaluated for efficient TgBI expression by immunofluorescence microscopy. 


\subsection{Indirect immunofluorescence assay}

HeLa and TgBIs-transfected HeLa cells were seeded in a 24-well plate with $13 \mathrm{~mm}$ round glass cover slips at a density of 75,000 cells per well. After 24 and 48 h, cells were washed once in PBS, fixed in $4 \%(\mathrm{w} / \mathrm{v})$ paraformaldehyde in $0.1 \mathrm{M}$ sodium cacodylate $\mathrm{pH} 7.4$ for $1 \mathrm{~h}$ at room temperature and were then incubated for $10 \mathrm{~min}$ in $50 \mathrm{mM} \mathrm{NH}_{4} \mathrm{Cl}$ in $\mathrm{PBS}$ at room temperature. The cells were blocked/permeabilised with $0.1 \mathrm{mg} / \mathrm{ml}$ saponin and $1 \%$ in PBS (blocking solution) for $1 \mathrm{~h}$ at room temperature. After having been washed with 0.1 $\mathrm{mg} / \mathrm{ml}$ saponin in PBS (washing solution), cells were incubated with anti-FLAG antibody (diluted 1:800 in blocking solution) for $1 \mathrm{~h}$ at room temperature. The cells were then washed three times (10 min for each step) with washing solution before being incubated with Cy2-conjugated anti-mouse IgG antibody (diluted 1:400 in blocking solution) for $1 \mathrm{~h}$ at room temperature in the dark. Following three washing steps and a subsequent washing step in PBS for $10 \mathrm{~min}$, the cells were incubated in $5 \mu \mathrm{g} / \mathrm{ml}$ propidium iodide in PBS for 5 min in order to visualize the whole cell population. The samples were then washed twice (5 min each) in PBS, were shortly rinsed with $\mathrm{ddH}_{2} \mathrm{O}$, and finally mounted with Mowiol 4-88. The cells were analysed by confocal laser scanning microscopy with a $63 x$ oil-immersion objective.

\subsection{Colocalisation of TgBIs}

MitoTracker $^{\circledR}$ Orange CM- $\mathrm{H}_{2} \mathrm{TMRos}$ was employed for detecting a possible colocalisation of TgBIs with mitochondria in HeLa-TgBIs. To this end, $1.5 \times 10^{4} \mathrm{HeLa}$ or TgBIs-expressing HeLa cells per well were grown on $13 \mathrm{~mm}$ round glass cover slips in a 24-well plate. When cells have reached confluence, HeLa cell culture medium was replaced with pre-warmed DMEM without supplement but containing $500 \mathrm{nM}$ MitoTracker ${ }^{\circledR}$ probe for $45 \mathrm{~min}$ at $37^{\circ} \mathrm{C}$. After having been washed in DMEM for 5 min at $37^{\circ} \mathrm{C}$, cells were washed for $5 \mathrm{~min}$ with $1 \mathrm{x}$ PBS at room temperature before fixing them with $4 \%$ paraformaldehyde in $0.1 \mathrm{M}$ sodium cacodylate $\mathrm{pH} 7.4$ for $15 \mathrm{~min}$ at room temperature. Cells were then permeabilised using 0.25\% Triton X-100 in PBS for 15 min and were thereafter blocked with $1 \%$ BSA in PBS (blocking solution) for $1 \mathrm{~h}$. Cells were further incubated with anti-FLAG antibody (diluted 1:800 in blocking solution) for $1 \mathrm{~h}$ at room temperature. Cells were then washed three times (10 min for each step) with $1 \mathrm{x}$ PBS 
before being incubated with Cy2-conjugated anti-mouse IgG antibody (diluted 1:400 in blocking solution) for $1 \mathrm{~h}$ at room temperature in the dark. Following three washing steps as above, cells were shortly rinsed with $\mathrm{ddH}_{2} \mathrm{O}$ and finally mounted with Mowiol 4-88. The cells were analysed by confocal laser scanning microscopy with a $63 x$ oil-immersion objective.

\subsection{Measurement of caspase $3 / 7$ activity}

HeLa and TgBIs-expressing HeLa cells were seeded in $92 \mathrm{~mm}$ cell culture dishes $(1.5 \mathrm{x}$ $10^{6}$ cells per dish). On the following day, cells were treated with $0.3 \mu \mathrm{M}$ staurosporine or $10 \mu \mathrm{M}$ brefeldin A or were left untreated, and incubated for 24 and $48 \mathrm{~h}$. At the indicated time points, cells were then isolated by EDTA/trypsin treatment (see 2.2.1.3) and centrifuged at $400 \mathrm{x} \mathrm{g}$ for $5 \mathrm{~min}$. After having been washed in PBS, cells were transferred into microcentrifuge tubes and centrifuged again at 3,400 $\mathrm{xg}(6,000 \mathrm{rpm})$ for $3 \mathrm{~min}$ at $4^{\circ} \mathrm{C}$. Pelleted cells were lysed in $50 \mu \mathrm{l}$ NP-40 buffer (see below) supplemented with $4 \%$ protease inhibitor cocktail on ice for $15 \mathrm{~min}$. The supernatants were collected by pelleting insoluble material at $18,400 \mathrm{x} \mathrm{g}(14,000 \mathrm{rpm})$ for $5 \mathrm{~min}$ at $4^{\circ} \mathrm{C}$. They were stored at $-80^{\circ} \mathrm{C}$ until use or directly subjected to a caspase activity assay.

To this end, an equal amount of proteins from each sample was applied for the activity assay; protein concentrations were measured as described below (see section 2.2.4.4.5). After adjusting an equal protein concentration in all samples, $10 \mu 1$ of supernatant was pipetted into the wells of a 96-well plate, and then mixed with $90 \mu 1$ reaction buffer containing DEVD-AMC, i.e. the substrate of caspase 3 or 7. Each sample was prepared in triplicate and NP-40 buffer was used as a blank. Cleavage of the substrate was measured using a multi-label microplate reader at $37^{\circ} \mathrm{C}$ at excitation and emission wavelengths of 380 and $460 \mathrm{~nm}$, respectively. The fluorescence was recorded every $5 \mathrm{~min}$ during a period of $60 \mathrm{~min}$. The caspase activity was assessed by calculating the increase in the fluorescence intensity from 5 to $60 \mathrm{~min}$. 
NP-40 lysis buffer $\mathrm{pH} 8.0$

$150 \mathrm{mM} \mathrm{NaCl}$

$50 \mathrm{mM}$ Tris base

$1 \%$ Nonidet P40

$\underline{\text { MDB buffer }}$

10 mM HEPES pH 7.0

$40 \mathrm{mM} \beta$-glycerophosphate

$50 \mathrm{mM} \mathrm{NaCl}$

$2 \mathrm{mM} \mathrm{MgCl}{ }_{2}$

5 mM EGTA $\underline{\text { Reaction buffer }}$

$0.1 \mathrm{mg} / \mathrm{ml}$ BSA (in MDB buffer)

$0.1 \%$ CHAPS (in MDB buffer)

$10 \mu \mathrm{M}$ DEVD-AMC

\subsection{Quantification of protein concentration}

Protein concentrations were measured in a microplate format following the instructions from the $\mathrm{BCA}^{\mathrm{TM}}$ Protein Assay Kit. In brief, $25 \mu \mathrm{l}$ of each BSA standard (provided in the kit as stock solution with a concentration of $2 \mathrm{mg} / \mathrm{ml}$ ) with known concentration, ranging from 125 to $1,500 \mu \mathrm{g} / \mathrm{ml}$ (stock solution of BSA was diluted in NP-40 lysis buffer), or unknown samples were transferred in duplicate into the wells of a 96-well plate. Thereafter, $200 \mu \mathrm{l}$ of working reagent (prepared by mixing 50 parts of reagent $\mathrm{A}$ with 1 part of reagent B) were added to each well and the plate was mixed thoroughly on a plate shaker for $30 \mathrm{sec}$. Samples were then incubated for $30 \mathrm{~min}$ at $37^{\circ} \mathrm{C}$ before measuring the optical density in a microplate reader at $550 \mathrm{~nm}$. The protein concentrations of unknown samples were calculated using a standard curve equation.

\subsection{Analysis of hypoploid DNA}

To quantitatively measure apoptotic nuclei, staining with propidium iodide and flow cytometry have been performed. For this purpose, HeLa and TgBIs-expressing HeLa cells were seeded in a 6 -well plate $\left(2.5 \times 10^{5}\right.$ cells per well) overnight. They were then treated with $0.3 \mu \mathrm{M}$ staurosporine or $10 \mu \mathrm{M}$ brefeldin $\mathrm{A}$ or were left untreated. Cells were harvested at 24 and $48 \mathrm{~h}$ post treatment by centrifugation at $400 \mathrm{x} \mathrm{g}$ for $5 \mathrm{~min}$. Pelleted cells were resuspended in $300 \mu \mathrm{l}$ PBS containing $5 \%$ FCS, permeabilised by adding 750 
$\mu \mathrm{l}$ ice-cold $70 \%$ ethanol and incubated for $10 \mathrm{~min}$ at $4^{\circ} \mathrm{C}$. After cells have been washed with PBS, $50 \mu \mathrm{g} / \mathrm{ml}$ propidium iodide in PBS and $0.5 \mathrm{mg} / \mathrm{ml}$ RNase A were added and the cells were incubated for additional $20 \mathrm{~min}$ at room temperature in the dark. Stained cells were then centrifuged as above, washed in $500 \mu \mathrm{PBS}$ and finally resuspended in 500-750 $\mu 1$ PBS. At least 10,000 cells of each sample were analysed by flow cytometry (FACSCalibur).

\subsection{Redistribution of TgBIs during cell death induction in transgenic HeLa cells}

HeLa and TgBIs-expressing HeLa cells were seeded on $13 \mathrm{~mm}$ round glass cover slips in a 24 -well plate $(75,000$ cells per well). Cells were treated with $0.3 \mu \mathrm{M}$ staurosporine, or 10 $\mu \mathrm{M}$ brefeldin $\mathrm{A}$, or were left untreated for 24 and $48 \mathrm{~h}$. At the indicated time points, cells were washed once in PBS, fixed in $4 \%(\mathrm{w} / \mathrm{v})$ paraformaldehyde in $0.1 \mathrm{M}$ sodium cacodylate $\mathrm{pH} 7.4$ for $1 \mathrm{~h}$ at room temperature and were then blocked/permeabilised in 0.1 $\mathrm{mg} / \mathrm{ml}$ saponin and $1 \%$ in PBS (blocking solution) for $1 \mathrm{~h}$ at room temperature. Staining of TgBIs using anti-FLAG primary antibody and Cy2-conjugated secondary antibody was carried out as described in section 2.2.4.3.2.

\subsubsection{Putative T. gondii Endonuclease G (TgEndoG)}

\subsection{Cloning procedure}

\subsection{Digestion of plasmid or PCR products}

The TgEndoG coding sequence (TgME49_008710) was released from pBluescript SKTgEndoG recombinant plasmid (synthetically generated, purchased from ATG:biosynthetics $\mathrm{GmBH}$ ) by digestion with EcoRI and KpnI and was subcloned into EcoRI- and KpnI-digested pASK-IBA3 plus vector. Digestion was performed by incubating $0.5 \mu \mathrm{g}$ of plasmid DNA, $10 \mathrm{U}$ of each enzyme, buffer 1 , and $100 \mu \mathrm{g} / \mathrm{ml}$ BSA at $37^{\circ} \mathrm{C}$ overnight.

Alternatively, TgEndoG coding sequence was amplified from the pASK-IBA3-TgEndoG recombinant vector using primer pairs (see Table 2.6) which introduced appropriate restriction enzyme sites; SpHI and HindIII for cloning into pQE30 or EcoRI and HindIII for cloning into pRSET-A. Amplification was carried out by incubating (final 
concentration) 1 x buffer, $1.5 \mathrm{mM} \mathrm{MgSO}_{4}, 0.2 \mathrm{mM}$ of each dNTP, $0.3 \mu \mathrm{M}$ of each forward and reverse primer, $5 \%(\mathrm{v} / \mathrm{v})$ DMSO, $1 \mu \mathrm{l}$ plasmid, $0.02 \mathrm{U}$ KOD DNA polymerase and $\mathrm{ddH}_{2} \mathrm{O}$ to give a final volume of $25 \mu$. The target sequence was amplified by PCR using the following cycling program: (i) denaturation at $95^{\circ} \mathrm{C}$ for $2 \mathrm{~min}, 40$ cycles of (ii) denaturation at $95^{\circ} \mathrm{C}$ for $20 \mathrm{sec}$, (iii) annealing at $60^{\circ} \mathrm{C}$ for $10 \mathrm{sec}$, (iv) extention at $70^{\circ} \mathrm{C}$ for $1 \mathrm{~min}$, and (v) a final extention at $70^{\circ} \mathrm{C}$ for $5 \mathrm{~min}$. PCR products were purified using the QIAquick PCR purification kit (Roche) as described in the manufacturer's protocol. Ten $\mu 1$ of purified PCR product was then double-digested by adding $1 \mu \mathrm{l}$ of $\mathrm{SpHI}$ and HindIII, and buffer 2 in a total volume of $20 \mu$ l. Digestion with EcoRI and HindIII was done in two successive steps: the PCR product was first digested with HindIII (with appropriate buffer), gel-purified (see below) and afterward digested with EcoRI. Digestion was performed at $37^{\circ} \mathrm{C}$ overnight.

\subsection{Analysing DNA fragments by agarose gel electrophoresis}

PCR products or digested plasmids $(5-10 \mu \mathrm{l})$ were mixed with 6 x DNA loading dye (Fermentas) and separated in 0.8-1\% agarose in $1 \mathrm{x}$ TAE contained $1 \mu \mathrm{g} / \mathrm{ml}$ ethidium bromide. DNA markers were separated in parallel with the samples. Bands were visualised under ultraviolet light illumination and documented using a BioDoc II digital imaging system (Biometra).

\section{$\underline{\text { TAE buffer }}$}

$40 \mathrm{mM}$ Tris base

$1 \%$ glacial acetic acid

1 mM EDTA

\subsection{DNA extraction from agarose gels}

DNA was extracted from agarose gels using the QIAquick PCR Purification kit according to the manufacturer's protocol. Briefly, DNA was separated in a $1 \%$ agarose gel in $1 \mathrm{x}$ TAE contained $1 \mu \mathrm{g} / \mathrm{ml}$ ethidium bromide. DNA bands of interest were visualised and excised with a scalpel under ultraviolet light illumination. Three volumes of buffer QG were then added to 1 volume of the excised gel. The gel was incubated at $50^{\circ} \mathrm{C}$ for $10 \mathrm{~min}$ 
(or until the gel was completely dissolved). The mixture was applied to a QIAquick spin column placed in a 2-ml collection tube. DNA was bound to the membrane by centrifugation at 17,900 x g (13,000 rpm) for $1 \mathrm{~min}$ and washed with $750 \mu \mathrm{l}$ of Buffer PE. Finally, DNA was eluted by adding $50 \mu \mathrm{l}$ buffer EB to the centre of the column and collected by centrifugation.

\subsection{Vector dephosphorylation}

To avoid vector re-circularisation during cloning, the digested vector was dephosphorylated by incubating gel-purified vector with $1 \mathrm{x}$ buffer and $10 \mathrm{U}$ Antarctic phosphatase for $1 \mathrm{~h}$ at $37^{\circ} \mathrm{C}$. Thereafter, the phosphatase was heat-inactivated for $5 \mathrm{~min}$ at $60^{\circ} \mathrm{C}$.

\subsection{Ligation}

Dephosphorylated vector and insert were mixed in different ratios of vector to insert $(1: 1$, 1:3, 1:5), and were incubated with $1 \mu \mathrm{T} 4$ DNA ligase (400 U), $2 \times$ buffer and $\mathrm{ddH}_{2} \mathrm{O}$ to give a total volume of $20 \mu \mathrm{l}$. The ligation reaction was incubated in a thermocycler at decreasing temperatures of $22^{\circ} \mathrm{C}$ for $2 \mathrm{~h}, 18^{\circ} \mathrm{C}$ for $1 \mathrm{~h}, 14^{\circ} \mathrm{C}$ for $1 \mathrm{~h}, 10^{\circ} \mathrm{C}$ for $1 \mathrm{~h}, 6^{\circ} \mathrm{C}$ for 1 $\mathrm{h}$, and was then incubated at $4{ }^{\circ} \mathrm{C}$ overnight. The ligation reaction mixture ( 5 to $7 \mu \mathrm{l}$ ) was then transformed into E. coli DH5 $\alpha$.

\subsection{Transformation}

E. coli $\mathrm{DH} 5 \alpha$ (or BL21) competent cells were thawed on ice for $5 \mathrm{~min}$, mixed carefully with 1-50 ng plasmid DNA, and incubated for $30 \mathrm{~min}$ on ice. Transformation of recombinant plasmid DNA was done by heat shock at $42^{\circ} \mathrm{C}$ for exactly $55 \mathrm{sec}$ to transform E. coli $\mathrm{DH} 5 \alpha$ or for $90 \mathrm{sec}$ for transforming ligation reaction mixture (see above) into $E$. coli DH5a. Transformation of recombinant plasmid DNA into E. coli BL21 or E. coli BL21 codon plus was performed by heat shock at $42^{\circ} \mathrm{C}$ for exactly $90 \mathrm{sec}$. After adding $500 \mu \mathrm{l}$ of LB medium (without antibiotic), the cells were incubated at $37^{\circ} \mathrm{C}$ for $1 \mathrm{~h}$ with shaking at $300 \mathrm{rpm}$. Different amounts of cells were plated $(50 \mu 1,100 \mu 1,150 \mu \mathrm{l}$, and 200 $\mu 1$ of transformed cells) onto LB plates (with appropriate antibiotics for selection of transformants) and the plates were incubated upside down at $37^{\circ} \mathrm{C}$ overnight. 
$\underline{\mathrm{LB} \text { medium for } 1 \mathrm{~L}}$

$5 \mathrm{~g}$ yeast extract

$10 \mathrm{~g} \mathrm{NaCl}$

$10 \mathrm{~g}$ tryptone
$\underline{\mathrm{LB}}$ plate for $1 \mathrm{~L}$

$5 \mathrm{~g}$ yeast extract

$10 \mathrm{~g} \mathrm{NaCl}$

$10 \mathrm{~g}$ tryptone

$15 \mathrm{~g}$ agar

\subsection{Screening transformants}

Selection of positive transformants was done by colony PCR, plasmid digestion or sequencing. Using an inoculation loop, single colonies were picked and stirred in $20 \mu \mathrm{l}$ of $20 \mathrm{mM} \mathrm{NaOH}$ in a microcentrifuge tube. In order to extract the plasmid, the cells were heated for $15 \mathrm{~min}$ at $95^{\circ} \mathrm{C}$ and centrifuged at $12,000 \mathrm{rpm}$ for $5 \mathrm{~min}$ to completely collect all solution. For PCR amplification, the following reaction mixture was set up (final concentrations): $1 \mathrm{x}$ buffer, $1.5 \mathrm{mM} \mathrm{MgSO} 4,0.2 \mathrm{mM}$ of each dNTP, $0.3 \mu \mathrm{M}$ each primers TgEndoG_4_forward and reverse, $5 \%$ (v/v) DMSO, $1 \mu$ l plasmid, 0.02 U KOD DNA polymerase and $\mathrm{ddH}_{2} \mathrm{O}$ to give a final volume of $25 \mu \mathrm{l}$. The DNA target was amplified as follows: (i) denaturation at $95^{\circ} \mathrm{C}$ for $2 \mathrm{~min}, 45$ cycles of (ii) denaturation at $95^{\circ} \mathrm{C}$ for 20 sec, (iii) annealing at $60^{\circ} \mathrm{C}$ for $10 \mathrm{sec}$, and (iv) extention at $70^{\circ} \mathrm{C}$ for $15 \mathrm{sec}$, and (v) a final extention at $70^{\circ} \mathrm{C}$ for $5 \mathrm{~min}$, followed by cooling at $4^{\circ} \mathrm{C}$ until the end of the PCR.

Restriction enzyme digestion of recombinant plasmids was also performed in order to confirm transformation with the correct vector. The restriction enzymes used depended on the vectors: EcoRI and KpnI for pASK-IBA3 plus, SpHI and HindIII for pQE30, or EcoRI and HindIII for pRSET-A. PCR products and digested plasmids were verified by agarose gel electrophoresis as described above (see 2.2.4.4.1.2).

Positive clones were further confirmed by DNA sequencing (Seqlab, Göttingen; www.seqlab.de.).

\subsection{Plasmid extraction}

Selected single colonies were inoculated into $5 \mathrm{ml}$ LB medium supplemented with antibiotics for selection of transformants and grown overnight at $37^{\circ} \mathrm{C}$. Plasmid DNA was isolated from overnight culture using the GenElute Plasmid Mini Prep Kit according to the 
manufacturer's instructions. Large scale plasmid preparation was achieved by using the GenElute Plasmid Maxi Prep Kit as recommended by the manufacturer.

\subsection{Preparation of glycerol stocks of bacteria}

Five hundred $\mu$ l of overnight culture (in LB medium with appropriate antibiotic) was gently mixed with $500 \mu \mathrm{l}$ sterile $87 \%$ glycerol. After approximately $5 \mathrm{~min}$ at room temperature, cells were transferred to $-80^{\circ} \mathrm{C}$ for long-term storage.

\subsection{Protein expression}

To express TgEndoG recombinant protein, plasmids were transformed into E. coli BL21 or E. coli BL21 codon plus (see above) and cells were selected for ampicillin-resistant transformants. Transformants were streaked onto LB plates supplemented with antibiotic (ampicillin for BL21 or ampicillin and chlorampenicol for BL21 codon plus). Positive colonies were inoculated into 2-5 ml LB medium with appropriate antibiotics and grown overnight at room temperature with constant shaking. Overnight cultures were further used to inoculate $25-100 \mathrm{ml} \mathrm{LB}$ medium with antibiotics and grown at room temperature with shaking until reaching an $\mathrm{OD}_{600}=0.4-0.6$. Protein expression was induced by adding $25 \mathrm{ng}$ anhydrotetracycline (for pASK-IBA3 plus) or 1-2 mM IPTG (for pQE30 and pRSET-A). Induction was done for 2 hours. Cells were harvested by centrifugation at 4,500 x g for 12 $\min$ at $4^{\circ} \mathrm{C}$. Bacterial pellets were stored at $-20^{\circ} \mathrm{C}$.

\subsection{Analysis of protein expression}

Protein expression was assessed by performing SDS-PAGE and protein detection by Coomassie blue staining or Western blot analysis.

\subsection{Sodium Dodecyl Sulphate-Polyacrylamide Gel Electrophoresis (SDS- PAGE)}

Separation of proteins was performed by SDS-PAGE using the Mini Protean 3 (Biorad) system; $7.5 \%$ separating gels and $4.4 \%$ stacking gels were used in all experiments. The separating gel was prepared as described below, poured into a vertical gel assemble with spacers of $0.75 \mathrm{~mm}$, carefully overlaid with isopropanol and allowed to polymerise. 
Isopropanol was then removed by washing twice with $\mathrm{ddH}_{2} \mathrm{O}$ and a stacking gel was poured onto the separating gel and a comb was inserted. After polymerisation of the gel, samples were mixed with $2 \mathrm{x}$ sample buffer and heated for $5 \mathrm{~min}$ at $99^{\circ} \mathrm{C}$ in a thermomixer. A prestained protein marker was prepared in parallel. Electrophoresis was performed in an electrophoresis chamber with 1 x SDS running buffer at a constant current of $25 \mathrm{~mA}$ per gel.

Separating gel $(7.5 \%)$

$0.5 \mathrm{M}$ Tris- $\mathrm{HCl}(\mathrm{pH} 8.8)$

$0.1 \%(\mathrm{w} / \mathrm{v}) \mathrm{SDS}$

$7.5 \%$ acrylamide/bisacrylamide

$(30 \% / 0.8 \% \mathrm{w} / \mathrm{v})$

$0.1 \%(\mathrm{w} / \mathrm{v})$ APS

$0.04 \%$ TEMED

2x sample buffer

125 mM Tris-Hcl pH 6.8

$4 \%(\mathrm{w} / \mathrm{v}) \mathrm{SDS}$

$20 \%(\mathrm{v} / \mathrm{v})$ glycerol

$65 \mathrm{mM}$ DTT

$0.002 \%$ bromophenol blue
Stacking gel $(4.4 \%)$

0.125 M Tris- $\mathrm{HCl}$ (pH 6.8)

$0.2 \%(\mathrm{w} / \mathrm{v}) \mathrm{SDS}$

$4.4 \%$ acrylamide/bisacrylamide

$(30 \% / 0.8 \% \mathrm{w} / \mathrm{v})$

$0.04 \%(w / v)$ APS

$0.4 \%$ TEMED

$0.004 \%$ (w/v) Bromophenol blue

10x SDS running buffer $(1 \mathrm{~L})$

$0.25 \mathrm{M}$ Tris- $\mathrm{HCl}$

1.92 M Glycin

$1 \%(\mathrm{w} / \mathrm{v}) \mathrm{SDS}$

\subsection{Coomassie blue staining of proteins separated by SDS-PAGE}

Polyacrylamide gels were fixed in $8.5 \%$ o-phosphoric acid and $20 \%$ absolute methanol for $1 \mathrm{~h}$ at room temperature with constant shaking. Gels were then submerged in colloidal Coomassie blue solutions (see below) at room temperature overnight. After having been washed three times $\left(10 \mathrm{~min}\right.$ each) in $25 \%$ absolute methanol, gels were soaked in $\mathrm{ddH}_{2} \mathrm{O}$. The gels were then encased in plastic wraps and dried at room temperature overnight. 


\section{Colloidal Coomassie blue solution}

$20 \%$ absolute methanol

$20 \%$ Roti $^{\circledR}$-Blue solution (Roth)

$60 \% \mathrm{ddH}_{2} \mathrm{O}$

\subsection{Electroblotting of proteins separated by SDS-PAGE}

Proteins separated by polyacrylamide gel electrophoresis were transferred onto a nitrocellulose membrane (NC, GE Healthcare) using a semidry blot system. For this purpose, the polyacrylamide gel and the NC membrane were placed within a stack of filter papers soaked in different transfer buffers as follows (from anode to catode): 6 Whatman filter papers soaked with $0.3 \mathrm{M}$ Tris- $\mathrm{HCl}(\mathrm{pH} 10.4)$ and $20 \%$ methanol, 3 Whatman filter papers soaked with $25 \mathrm{mM}$ Tris- $\mathrm{HCl}(\mathrm{pH} \mathrm{10.4)}$ and $20 \%$ methanol, Hybond nitrocellulose membrane, polyacrylamide gel, and 9 Whatman filter papers soaked with $40 \mathrm{mM} 6$ aminocapronic acid ( $\mathrm{pH}$ 7.6) and $20 \%$ methanol. Proteins were transferred to the NC membrane at room temperature with a constant current of $0.8 \mathrm{~mA} / \mathrm{cm}^{2}$ blot area for $1.5 \mathrm{~h}$.

\subsection{Blocking, washing and antibody incubation}

Transferred proteins were visualised by reversible staining with Ponceau S. Nitrocellulose membranes containing transferred protein was put in a petri dish and soaked in $0.1 \%(\mathrm{w} / \mathrm{v})$ Ponceau $\mathrm{S}$ in $5 \%(\mathrm{v} / \mathrm{v})$ acetic acid (Sigma-Aldrich) until proteins bands were clearly visible. After Ponceau S staining, the NC membrane was washed three times with water. For immunoblotting, nonspecific binding sites were blocked and proteins were stained, depending on the tag of the fusion protein, as follows.

For detection of His-tag fusion protein, $\mathrm{NC}$ membrane was washed in $\mathrm{ddH}_{2} \mathrm{O}$ twice $(5 \mathrm{~min}$ each) and was then incubated in blocking solution for $2 \mathrm{~h}$ at room temperature or overnight at $4^{\circ} \mathrm{C}$. After having been washed once in washing solution for $5 \mathrm{~min}$, the NC membrane was incubated with mouse anti-His-tag antibody (diluted 1:1,000 in blocking solution) for $2 \mathrm{~h}$ at room temperature (or overnight at $4^{\circ} \mathrm{C}$ ) and then washed three times 5 min with washing solution. Incubation of the NC membrane with anti-mouse IgG HRP-conjugated secondary antibody (diluted 1:2,000 in secondary antibody dilution buffer) was done for 
$1.5 \mathrm{~h}$ followed by three washing steps (10 min each) with washing solution. Proteins were then detected using enhanced chemiluminescence detection (ECL).

For detection of protein fused to a Strep-tag, NC membrane was washed twice (10 min each) with TBS buffer $\mathrm{pH} 7.5$ and then it was blocked in blocking solution for $1 \mathrm{~h}$ at room temperature or overnight at $4^{\circ} \mathrm{C}$. After having been washed twice (10 min each) with TBSTween/Triton and an additional washing step for 10 min with TBS buffer, the NC membrane was incubated with mouse anti-Strep-tag antibody (diluted 1:2,000 in blocking solution) for $1 \mathrm{~h}$ at room temperature (or overnight at $4^{\circ} \mathrm{C}$ ) and then washed again as above. Immune complexes were then labelled with anti-mouse IgG HRP-conjugated secondary antibody (diluted 1:2,000 in secondary antibody dilution buffer) for $1 \mathrm{~h}$. Enhanced chemiluminescence detection was performed after washing the NC membrane four times (10 min each) with TBS-Tween/Triton.

\section{For detection of His-tag fusion protein For detection of Strep-tag fusion protein \\ Washing solution \\ $0.05 \%$ Tween-20 \\ $1 \times$ PBS \\ TBS buffer $\mathrm{pH} 7.5$ \\ $10 \mathrm{mM}$ Tris-Cl \\ $150 \mathrm{mM} \mathrm{NaCl}$}

Blocking solution

$5 \%$ dry skimmed milk

$0.2 \%$ Tween-20

$0.02 \%$ sodium azide

$1 \times$ PBS

$\underline{\text { Secondary antibody dilution buffer }}$

$5 \%$ dry skimmed milk

$0.05 \%$ Tween-20

$1 \times$ PBS
TBS-Tween/Triton $\mathrm{pH} 7.5$

$20 \mathrm{mM}$ Tris- $\mathrm{Cl}$

$500 \mathrm{mM} \mathrm{NaCl}$

$0.05 \%(\mathrm{v} / \mathrm{v})$ Tween

$0.02 \%(\mathrm{v} / \mathrm{v})$ Triton

\section{Blocking solution}

$3 \% \mathrm{BSA}(\mathrm{w} / \mathrm{v})$ in TBS

$\underline{\text { Secondary antibody dilution buffer }}$

$10 \%$ (w/v) dry skimmed milk in TBS 


\subsection{Protein Detection}

ECL reagents (GE Healthcare) were mixed 1:2 and used to incubate the membrane for 1 min. Superfluous solution was removed and the membrane was covered using a transparent plastic foil. Protein visualisation was done by exposing the membrane to a chemiluminescence imaging device (Luminescent Image Analyzer LAS-4000, Fujifilm). Images were recorded either by automatic or increment (up to $1 \mathrm{~h}$ ) exposure.

\subsubsection{Regulation of Toxoplasma PCD}

\subsubsection{Detection of $\Delta \Psi \mathrm{m}$}

Intact mitochondrial membrane potential $(\Delta \Psi \mathrm{m})$ is an indicator of vital cells. To label mitochondria with an intact $\Delta \Psi \mathrm{m}$, MitoTracker staining was used in this study. To this end, $2 \times 10^{7}$ parasites were cultivated in the presence of $10 \mu \mathrm{M}$ staurosporine or $20 \mu \mathrm{M}$ miltefosine to induce cell death for 24 and $48 \mathrm{~h}$. Non-treated parasites were used as controls. The parasites were then isolated by centrifugation at $1,800 \mathrm{x} \mathrm{g}$ for $10 \mathrm{~min}$. Pelleted cells were resuspended in $500 \mu \mathrm{l}$ pre-warmed RPMI containing $500 \mathrm{nM}$ MitoTracker ${ }^{\circledR}$ Orange CM-H $\mathrm{H}_{2} \mathrm{TMRos}$ for $45 \mathrm{~min}$ at $37^{\circ} \mathrm{C}$. The cells were fixed in $4 \%$ paraformaldehyde in $0.1 \mathrm{M}$ sodium cacodylate for $15 \mathrm{~min}$ at room temperature and were then centrifuged at $1,800 \mathrm{x} \mathrm{g}$ for $10 \mathrm{~min}$. After having been washed in $1 \mathrm{x}$ PBS by centrifugation as above, pelleted cells were resuspended in $10 \mu \mathrm{PBS}$, mixed with $10 \mu 1$ Mowiol 4-88, spread onto glass slides and covered with glass cover slips. Numbers of parasites with an intact mitochondrial membrane potential were counted from at least 500 cells using a confocal laser scanning microscopy with a 63x oil-immersion objective.

\subsubsection{In situ measurement of caspase-like activity}

CaspaTag assay, a method for labelling active caspases in situ, and flow cytometry were used in this study to determine the involvement of caspase-like proteins in the regulation of apoptosis-like cell death in T. gondii. Parasites were cultivated in a 24-well tissue culture plate $\left(2 \times 10^{7}\right.$ parasites per well) for $2,4,24$ and $48 \mathrm{~h}$ in the presence of $10 \mu \mathrm{M}$ staurosporine or $20 \mu \mathrm{M}$ miltefosine to induce cell death. Non-treated parasites were processed in parallel and were used as controls. The labelling of active caspase-like proteases was performed using the CaspaTag ${ }^{\mathrm{TM}}$ Pan-Caspase In Situ Assay Kit, 
Fluorescein (Chemicon International), following the manufacturer's recommendation. Briefly, at the indicated time points, $300 \mu \mathrm{l}$ of each sample were transferred into a $15-\mathrm{ml}$ tube and incubated with $10 \mu \mathrm{l}$ freshly prepared $30 \mathrm{x}$ FLICA solutions for $1 \mathrm{~h}$ at $37^{\circ} \mathrm{C}$ under $5 \% \mathrm{CO}_{2}$. One vial FLICA reagent (a carboxyfluorescein-labelled FAM-VAD-FMK) provided in the kit was dissolved in $50 \mu \mathrm{l}$ DMSO to give a $150 \mathrm{x}$ stock solution. Working solution $(30 \mathrm{x})$ was prepared by diluting the stock solution in PBS pH 7.4. After incubation with FLICA solution, $2 \mathrm{ml}$ of $1 \mathrm{x}$ wash buffer (diluted from $10 \mathrm{x}$ stock solution provided in the kit with $\mathrm{ddH}_{2} \mathrm{O}$ ) were mixed with each sample. Cells were then centrifuged at $400 \mathrm{xg}$ for $5 \mathrm{~min}$ at room temperature. Pelleted cells were resuspended in $1 \mathrm{ml}$ of $1 \mathrm{x}$ wash buffer and thereafter were centrifuged again as above. Parasites were then resuspended in $400 \mu 1$ $1 \mathrm{x}$ wash buffer. Measurement of caspase labelling was done using flow cytometry (FACSCalibur) from 100,000 events.

\subsubsection{Impact of proteases during induction of apoptosis-like cell death in $T$. gondii}

To evaluate the effect of protease inhibitors in the regulation of $T$. gondii apoptosis-like cell death, $2 \times 10^{7}$ parasites were seeded in a 24 -well plate and were incubated in the presence of $100 \mu \mathrm{M}$ Z-VAD-FMK or $100 \mu \mathrm{M}$ E-64 for $1 \mathrm{~h}$ prior to the treatment with 10 $\mu \mathrm{M}$ staurosporine or $20 \mu \mathrm{M}$ miltefosine. After $48 \mathrm{~h}$ of treatment, the parasites were isolated by centrifugation at $1,800 \mathrm{x} g$ for $10 \mathrm{~min}$ and pelleted parasites were resuspended in $1 \mathrm{ml}$ of $1 \mathrm{x}$ PBS. Parasite solutions (30-50 $\mu \mathrm{l})$ were then spread onto slides and were fixed in $4 \%(\mathrm{w} / \mathrm{v})$ paraformaldehyde in $0.1 \mathrm{M}$ sodium cacodylate $\mathrm{pH} 7.4$ for $1 \mathrm{~h}$ at room temperature. Blocking/permeabilisation were done by incubating cells in $0.1 \mathrm{mg} / \mathrm{ml}$ saponin and $1 \%$ BSA in PBS and afterwards, parasites were stained with rabbit anti- $T$. gondii antibody and TUNEL as described in section 2.2.2.1. The number of TUNELpositive parasites was counted from 500-800 cells using confocal laser scanning microscopy with a $63 x$ oil-immersion objective. 


\subsubsection{Bioinformatics}

The Toxoplasma gondii genome database can be accessed through www.toxodb.org. Tools available in the National Center for Biotechnology Information (NCBI: www.ncbi.nlm.nih.gov) or EMBL-EBI (www.ebi.ac.uk) was used for nucleic acid or protein alignment. Primer3, a primer design tool, was applied to design primers for DNA amplification. Prediction of TgEndoG structure was performed by using PyMOL Molecular Graphics System, Version 1.3r1-edu-Win32 Schrödinger, LLC (collaboration with Antonio Jiménez-Ruiz, Madrid). MitoProt was used for predicting a mitochondrial localisation of TgEndoG and TgBIs. Membrane topology of TgBIs was predicted by using several tools: TMHMM2.0, SOSUI, Pfam, MEMSAT_SVM, MEMSAT3, iPSORT, SCAMPI, and TopPred 0.01.

\subsubsection{Statistical analysis}

Unless otherwise stated, results were depicted as means \pm S.E.M. of at least three independent experiments. Comparisons were performed by Student's $t$-test; $p$-values of less than 0.05 were considered significant. 


\section{CHAPTER 3}

RESULTS

\subsection{Toxoplasma gondii exhibited markers of apoptosis in response to pro-apoptotic stimuli}

Here we describe multiple apoptosis markers which were identified in T. gondii upon treatment with pro-apoptotic stimuli.

\subsubsection{The occurrence of DNA fragmentation in $T$. gondii after stimulation of cell death}

One biochemical hallmark associated with apoptosis is DNA breakdown (Elmore, 2007; Popov and Korochkin, 2004). Terminal deoxynucleotidyl transferase-mediated XdUTP nick end labelling (TUNEL) assay followed by flow cytometry was used for analysing DNA strand breaks in $T$. gondii following treatment with diverse pro-apoptotic stimuli. The TUNEL reaction labels free 3'-OH termini of DNA fragments occurring during apoptosis with fluorochrome-conjugated nucleotides. In our study, $\mathrm{H}_{2} \mathrm{O}_{2}$, staurosporine, miltefosine, and camptothecin were used to trigger $T$. gondii death. Apoptosis can be induced by reactive oxygen species. It has been described that high concentration of $\mathrm{H}_{2} \mathrm{O}_{2}$ triggered necrosis whereas low or moderate concentration of $\mathrm{H}_{2} \mathrm{O}_{2}$ induced apoptosis; the effective $\mathrm{H}_{2} \mathrm{O}_{2}$ concentration depends on the cell model used for the experiments (Troyano et al., 2003). Staurosporine, an alkaloid isolated from Streptomyces, is widely used to induce apoptosis. It inhibits a broad spectrum of protein kinases (Bertrand et al., 1994). Miltefosine (hexadecylphosphocholine/HePC) was initially used as an anticancer drug. Recently, it has been proven as a potent chemotherapeutic agent against Leishmania spp.. Miltefosine is able to induce apoptosis in a variety of tumour cell lines by affecting membrane fluidity, but the exact molecular mechanism to initiate apoptosis remains unclear (Paris et al., 2004). Camptothecin is a DNA topoisomerase I inhibitor and effectively acts against a broad spectrum of tumours by initiating apoptosis (Liu et al., 2000). 
To this end, tachyzoites of $T$. gondii were cultivated in the presence of $\mathrm{H}_{2} \mathrm{O}_{2}$, staurosporine, miltefosine, or camptothecin for 24 and $48 \mathrm{~h}$. Freshly isolated and mocktreated parasites were used as controls. At the indicated time points, all samples were subjected to TUNEL staining. The number of TUNEL-positives parasites was quantitatively measured using flow cytometry (FACS). TUNEL-positive parasites were detected in freshly isolated populations $(\sim 20 \%)$ and this did not significantly increase during the next $48 \mathrm{~h}$ (Figure 3.1B), indicating a basal level of parasites with considerable DNA fragmentation. Parasites treated with pro-apoptotic stimuli displayed a higher percentage of TUNEL-positive cells to various extents as compared to freshly isolated and mock-treated parasites (Figure 3.1 A,B). Specifically, we observed a significant increase of the number of TUNEL-positive parasites after $\mathrm{H}_{2} \mathrm{O}_{2}$ treatment $(\sim 30 \%$ at $48 \mathrm{~h}$ post treatment; $\mathrm{p}<0.05)$, suggesting that reactive oxygen species may induce apoptosis in $T$. gondii. Staurosporine increased the number of TUNEL-positive parasites to approximately $50 \%$ and this was significantly higher as compared to the freshly isolated and control parasites $(\mathrm{p}<0.01)$. It indicates that protein phosphorylation may play critical roles in cell death regulation in $T$. gondii. Induction of TUNEL-positive parasites in our study was most prominently observed after miltefosine treatment (more than $55 \%$ at $48 \mathrm{~h}$ after treatment; $\mathrm{p}<0.01)$. Camptothecin also induced a significant number of TUNEL-positive parasites of approximately $40 \%$ after $48 \mathrm{~h}$ of treatment (Figure 3.1B; $<<0.05$ ). 
A

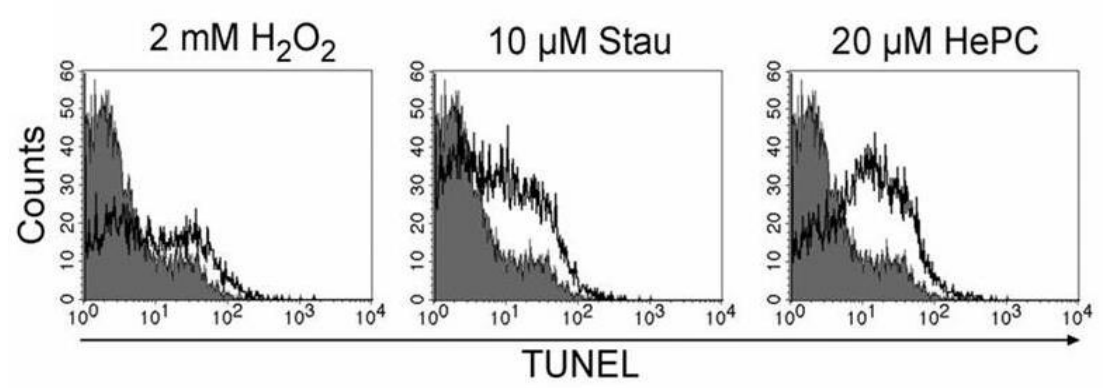

B

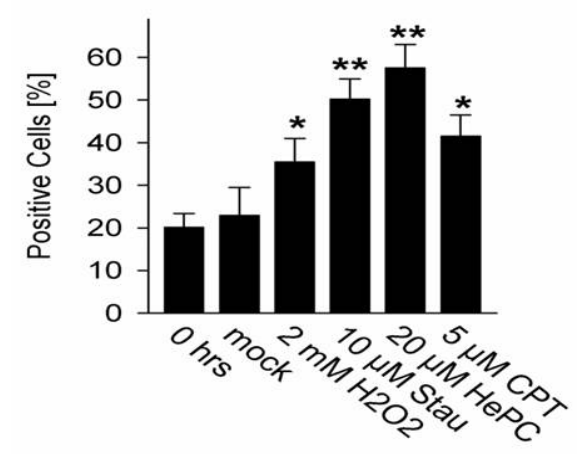

Figure 3.1. DNA fragmentation in extracellular parasites following treatment with pro-apoptotic stimuli was detected by TUNEL assay followed by flow cytometry. Extracellular parasites were treated with $2 \mathrm{mM} \mathrm{H}_{2} \mathrm{O}_{2}$, $10 \mu \mathrm{M}$ staurosporine (Stau), $20 \mu \mathrm{M}$ miltefosine (HePC), $5 \mu \mathrm{M}$ camptothecin (CPT), or were mock-treated. Freshly isolated parasites were prepared in parallel ( 0 hrs). A, Parasites were selected based on the FSC/SSC dot plots. Representative histograms of parasites treated for $48 \mathrm{~h}$ with $\mathrm{H}_{2} \mathrm{O}_{2}$, stau or $\mathrm{HePC}$ (open histograms) or of freshly isolated parasites (filled histograms) are depicted. B, Bars represent means \pm S.E.M. Results are from $5-6$ independent experiments (CPT: $\mathrm{n}=2)$. $* p<0.05 ; * * p<0.01$.

The strong induction of DNA strand breaks in $T$. gondii by staurosporine and miltefosine prompted us to use these cell death inducers for further experiments. Induction of cell death as detected by TUNEL-positive parasites was determined at $0,6,24,48$, and $72 \mathrm{~h}$ post staurosporine and miltefosine treatments. The time course experiments showed that staurosporine induced DNA fragmentation in a dose- and time-dependent manner. At the early time points (up to $48 \mathrm{~h}$ post treatment), the number of TUNEL-positive parasites between the lower concentration of staurosporine $(5 \mu \mathrm{M})$ and untreated (mock) parasites were not clearly different but it was considerably higher after $72 \mathrm{~h}$ of treatment with $5 \mu \mathrm{M}$ staurosporine as compared to control. Induction of DNA strand breaks in T. gondii was obviously increased by $10 \mu \mathrm{M}$ staurosporine from $48 \mathrm{~h}$ onwards until $72 \mathrm{~h}$ of treatment 
(Figure 3.2A). Miltefosine induced DNA fragmentation more rapidly than after staurosporine treatment. Increased levels of TUNEL-positive parasites were already clearly observed at $24 \mathrm{~h}$ post treatment and further increased thereafter (Figure 3.2B).

A

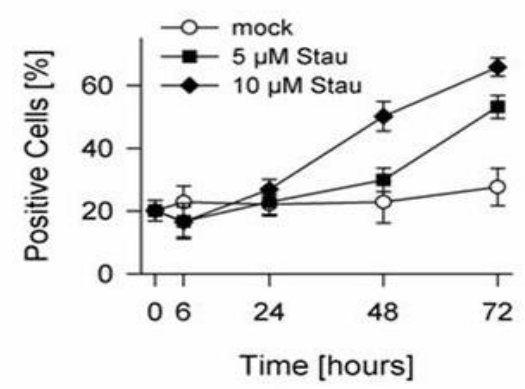

B

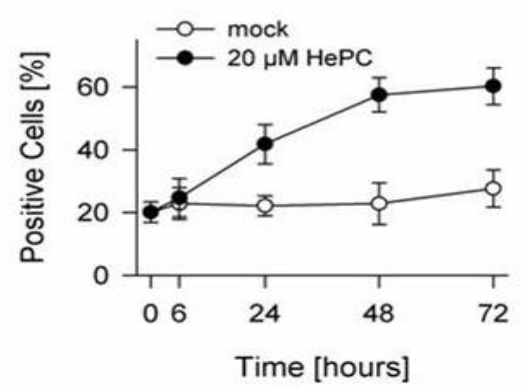

Figure 3.2. Kinetics of DNA fragmentation in $T$. gondii upon cell death induction by staurosporine or miltefosine as detected by TUNEL assay and flow cytometry. Extracellular parasites were treated with $5 \mu \mathrm{M}$ or $10 \mu \mathrm{M}$ staurosporine (Stau), $20 \mu \mathrm{M}$ miltefosine (HePC), or were mock-treated for up to $72 \mathrm{~h}$. Data represents means \pm S.E.M. from at least three independent experiments.

Together, the results indicated that DNA strand breaks, a hallmark of apoptosis, could be induced in $T$. gondii following treatment with common pro-apoptotic stimuli.

\subsubsection{Cell size reduction in extracellular parasites after treatment with pro- apoptotic stimuli}

Various morphological changes occur during apoptosis including cell shrinkage, i.e. pyknosis (Kerr et al., 1972). To investigate alteration in parasite size upon cell death induction, the means of the forward scatter (FSC) value as obtained by flow cytometry analysis of freshly isolated, untreated and treated parasites were compared; reduction in FSC value implied a decrease in cell size.

Our data showed that a reduction in cell size was observed in staurosporine- and miltefosine-treated parasites (Figure 3.3A). Staurosporine treatment led to a reduction of parasite size in a dose- and time-dependent fashion as likewise observed after TUNEL staining (see above) (Figure 3.3A). Such cell size reduction was clearly observed at $72 \mathrm{~h}$ post treatment. However, the reduction in cell size was more rapidly and more strongly detected in miltefosine-treated parasites (Figure 3.3A) that also displayed the highest percentage of TUNEL-positive parasites. 
A

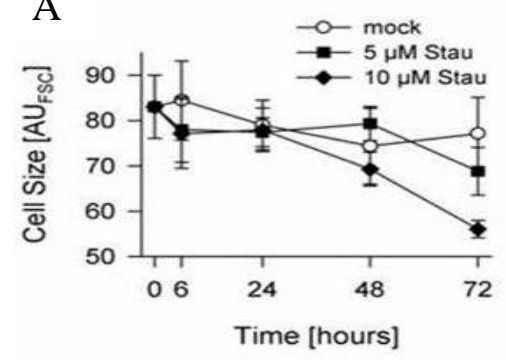

B

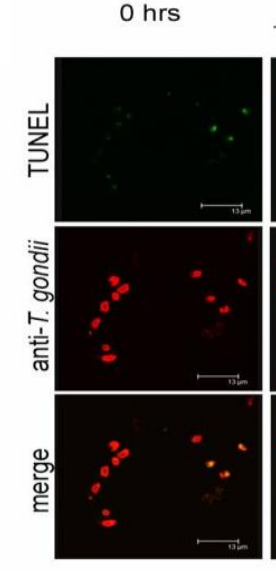

C

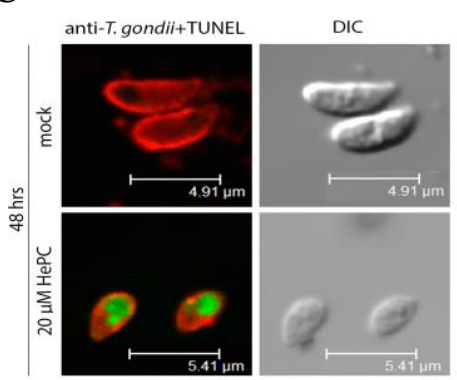

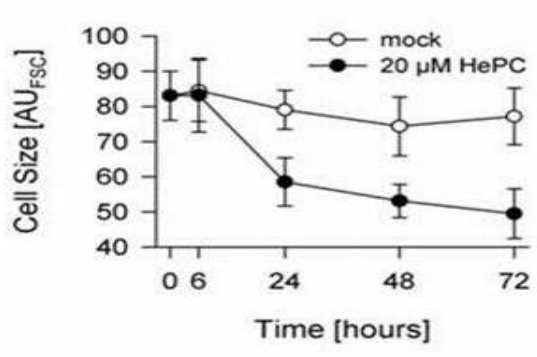

$48 \mathrm{hrs}$

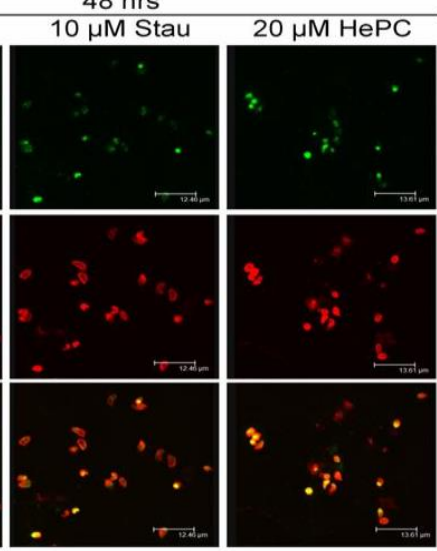

D

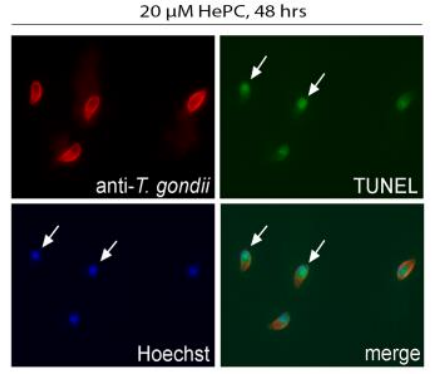

Figure 3.3. Reduction of the parasite cell size and alterations of the morphology of T. gondii following treatment with pro-apoptotic stimuli were assessed by flow cytometry and immunofluorescence microscopy. Parasites were treated with $5 \mu \mathrm{M}$ or $10 \mu \mathrm{M}$ staurosporine (Stau), $20 \mu \mathrm{M}$ miltefosine (HePC) or were mocktreated. A, Comparison of the parasite cell size between samples was based on the FSC value of FACS data analysis. Data represents means \pm S.E.M. from at least three independent experiments. B, Images are representative of freshly isolated (0 hrs), mock-treated, staurosporine (Stau)- or miltefosine (HePC)-treated parasites that have been stained with parasite-specific antibodies (red) and TUNEL reaction mix (green) at 48 $\mathrm{h}$ of treatment. C, Representative images of mock-treated or miltefosine (HePC)-treated parasites at $48 \mathrm{~h}$ post treatment as obtained by confocal laser scanning microscopy with high magnification clearly showed TUNEL-positive parasites after HePC treatment with a reduction in cell size. DIC, differential interference contrast. D, Hoechst staining (blue) of parasites treated with miltefosine (HePC) for $48 \mathrm{~h}$ confirmed the colocalisation of TUNEL labelling with nuclear staining (arrows). 
To further confirm and extend the results of TUNEL assay and flow cytometry, we performed TUNEL assay followed by confocal laser scanning microscopy. Parasites that had been treated or not with pro-apoptotic stimuli were stained with antibodies against $T$. gondii (red labelling) and thereafter were subjected to TUNEL staining (green labelling). Using confocal laser scanning microscopy, we observed the occurrence of DNA strand breaks in approximately $9 \%$ of freshly isolated and control parasites (data not shown). A strong increase in the number of TUNEL-positive parasites was detected following treatment with pro-apoptotic stimuli $(\sim 40 \%$ and $\sim 35 \%$ at $48 \mathrm{~h}$ post staurosporine and miltefosine treatment, respectively; Figure 3.3B). Moreover, TUNEL-positive tachyzoites showed morphologically a reduction in cell size; they became smaller and round in comparison to TUNEL-negative parasites that maintained their crescent shape (Figure 3.3B,C). To counterstain the nuclei of parasites, Hoechst 33258 (blue fluorescence) was applied after TUNEL staining. The results clearly confirmed a colocalisation of TUNEL labelling and Hoechst 33258 staining (Figure 3.3D, arrows), showing that DNA strand breaks occurred within the parasite nuclei.

Thus, we provided evidence that rounding-up of the cell and reduction of the cell volume, which are characteristic features of cells undergoing apoptosis, were also detected in $T$. gondii after treatment with pro-apoptotic stimuli.

\subsubsection{Treatment with staurosporine, but not HePC, triggered TUNEL-positivity in intracellular parasites}

To identify whether $T$. gondii would undergo apoptosis in the natural environment, i.e. intracellularly, confluent HFF cells were infected with tachyzoites of $T$. gondii for $24 \mathrm{~h}$ prior to treatment with staurosporine or miltefosine and then further incubated for up to 24 h. At 3, 6, and 24 h post treatment, the cells were fixed in paraformaldehyde and stained with parasite-specific antibodies and were subjected to TUNEL staining. The presence of TUNEL-positive intracellular parasites was then visualised by confocal laser scanning microscopy.

Staurosporine strongly triggered DNA strand breaks in $44.3 \%$ and $48.9 \%$ of intracellular parasites at 3 and $6 \mathrm{~h}$ post treatment, respectively. Since HFF cells were also affected by staurosporine (Figure 3.4, arrow) the occurrence of TUNEL-positive 
intracellular T. gondii could not be followed beyond the time point of $6 \mathrm{~h}$ of treatment. In contrast, miltefosine did not strongly induce DNA strand breaks in intracellular parasites (0.6 and $0.5 \%$ at 3 and $6 \mathrm{~h}$ after treatment, respectively; data not shown). Although TUNEL-positive intracellular parasites were not regularly detected in mock-treated cells, we also observed occasionally TUNEL-positive intracellular control parasites (data not shown).

This result established that treatment of intracellular $T$. gondii with a well-known pro-apoptotic stimulus, i.e. staurosporine, induces significant levels of DNA fragmentation, i.e. a hallmark of apoptosis.

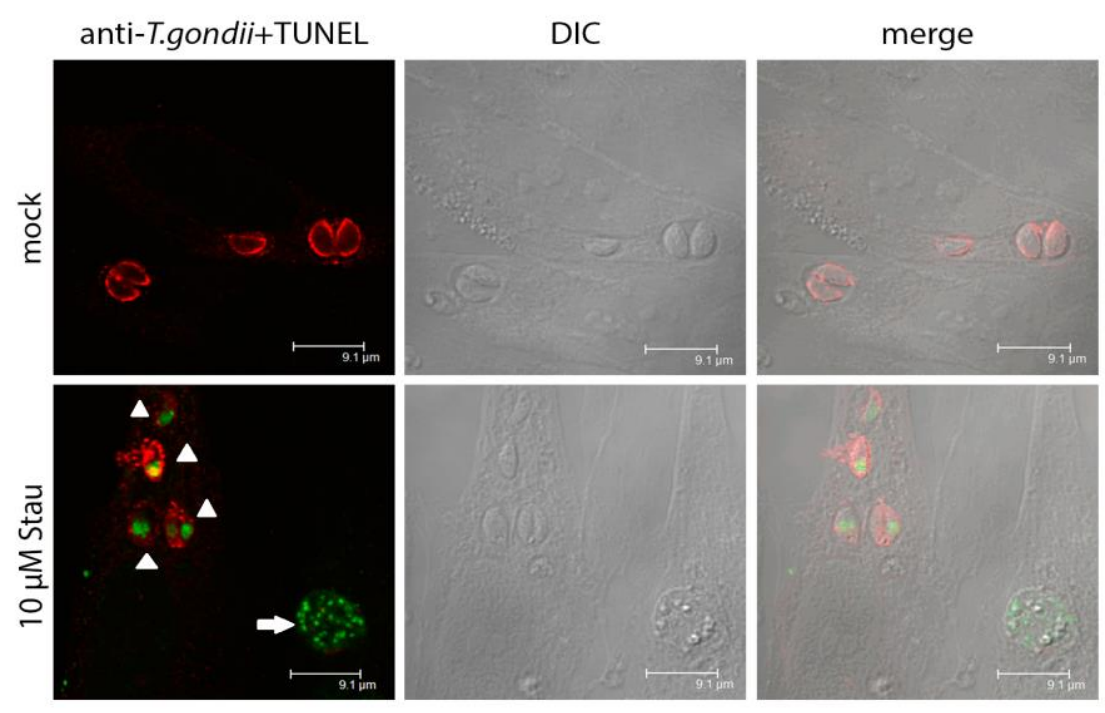

Figure 3.4. DNA fragmentation in intracellular parasites after treatment with staurosporine. Images represent staurosporine (stau)-treated or mock-treated T. gondii-infected HFF cells at $3 \mathrm{~h}$ post treatment. Note the appearance of TUNEL-positive intracellular parasites (arrowheads) and TUNEL-positive HFF cells (arrow). Differential interference contrast (DIC) images are included to assure intracellular location of the parasites.

\subsubsection{T. gondii displayed phosphatidylserine externalization following treatment with pro-apoptotic stimuli}

An important biochemical feature of apoptosis is phosphatidylserine (PS) exposure. Phosphatidylserine, a component of the cell membrane, is translocated from the inner leaflet to the outer leaflet of the plasma membrane during early stages of apoptosis; such PS-exposing cells are still viable (Martin et al., 1995; Zimmermann et al., 2001). The exposure of PS can be quantitatively detected using Annexin V, a $\mathrm{Ca}^{2+}$-dependent 
phospholipid binding protein with high affinity for PS (Vermes et al., 1995). 7-Aminoactinomycin (7-AAD), a standard flow cytometry viability probe, was included to distinguish between apoptotic and necrotic cells. Viable cells will exclude 7-AAD, whereas the membranes of dead and damaged cells are permeable to 7-AAD. Cells which undergo apoptosis will thus be stained positive for Annexin V-PE and negative for 7-AAD.

To this end, tachyzoites of $T$. gondii were incubated with staurosporine, miltefosine or were mock-treated for 24 and $48 \mathrm{~h}$. At the indicated time points, parasites were collected by centrifugation and were then incubated with Annexin V and 7-AAD. The stained cells were then subjected to flow cytometry. The results showed that approximately $5 \%$ of mock-treated parasites exposed PS on their cell membrane (Figure 3.5). Treatment of parasites with staurosporine and miltefosine resulted in a higher percentage of Annexin $\mathrm{V}$ positive/7-AAD negative cells at 24 and $48 \mathrm{~h}$ post treatment as compared to control parasites (Figure 3.5). Translocation of PS significantly increased at $24 \mathrm{~h}$ post staurosporine and miltefosine treatment with $\sim 12 \%$ and $\sim 17 \%$ of the cells being positive, respectively $(\mathrm{p}<0.01)$. This further increased slightly until $48 \mathrm{~h}$ after miltefosine treatment $(\mathrm{p}<0.01)$. Importantly, only minor percentages of 7-AAD positive cells were detected among control parasites and those treated with pro-apoptotic stimuli during the observation period ( 2 to $3 \%$; data not shown). This excluded the induction of necrosis following proapoptotic treatment of $T$. gondii.
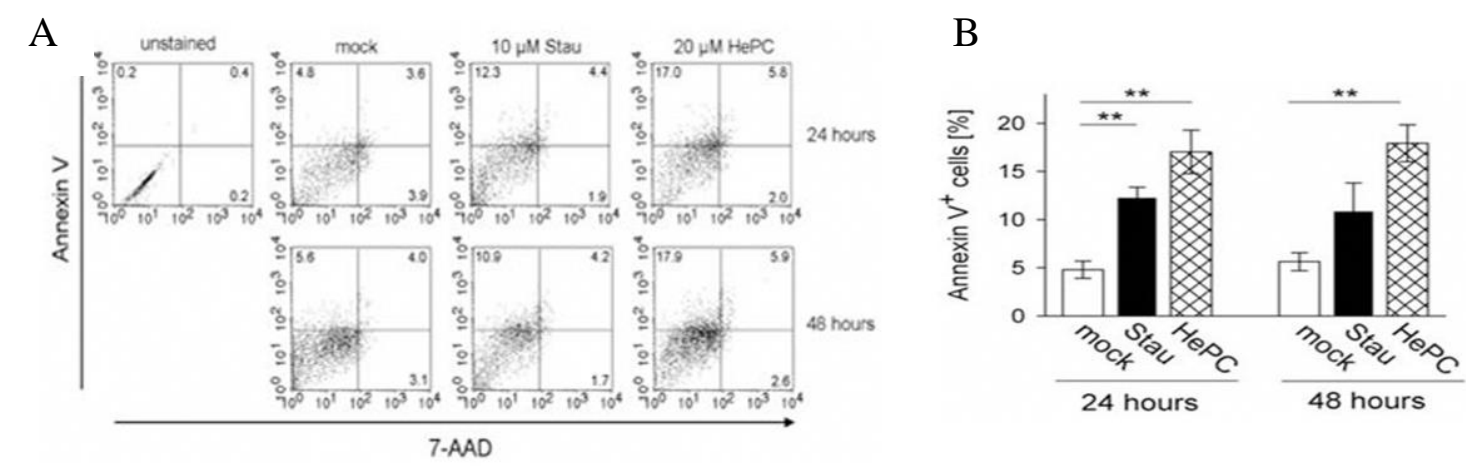

Figure 3.5. Translocation of phosphatidylserine (PS) after staurosporine or miltefosine treatment. The percentages of parasites which exposed PS on the outer leaflet of their plasma membrane after treatment with staurosporine (stau) or miltefosine (HePC) or mock treatment were assessed using Annexin V/7-AAD staining and by flow cytometry. Representative flow cytometry dot plots (A) and comparison between samples for the emergence of Annexin V-positive/7-AAD-negative parasites (B) are depicted. Bars represent means \pm S.E.M. from four independent experiments. $* * p<0.01$. 
Together, the data strongly indicated that following treatment with pro-apoptotic stimuli, T. gondii undergo a form of programmed cell death that shows characteristic features of apoptosis as observed in metazoan.

\subsubsection{Pre-treatment of extracellular parasites with pro-apoptotic stimuli diminished parasite invasiveness}

To evaluate whether extracellular parasites preserve their invasion ability after being pre-incubated with pro-apoptotic stimuli, extracellular tachyzoites were pre-treated with staurosporine or $\mathrm{HePC}$ or were mock-treated for $48 \mathrm{~h}$ and thereafter the parasites were used to inoculate confluent HFF cells. At 24 and $48 \mathrm{~h}$ of infection, cells were fixed and stained using parasite-specific antibodies and were then analysed using confocal laser scanning microscopy. The numbers of parasitophorous vacuoles (PV) were determined from 10 randomly selected visual fields. The results showed that untreated parasites were still able to invade HFF cells and to replicate as shown by the increasing sizes of parasitophorous vacuoles over time and numerous PVs per visual field (Figure 3.6A,B). A possible explanation for the survival of untreated tachyzoites in the extracellular milieu is that tachyzoites of $T$. gondii form mRNA aggregates with characteristics of stress granules (SG). The SG formation occurs when cells encounter environmental stress and it improves the fitness of extracellular parasites (Lirussi and Matrajt, 2011). Conversely, parasites which had been pre-treated with $10 \mu \mathrm{M}$ staurosporine or $20 \mu \mathrm{M}$ miltefosine lost their invasiveness; they resided extracellularly and were not able to invade host cells (Figure 3.6A). This indicated that extracellular parasites undergoing apoptosis might not be able to sustain a productive infection. 
A
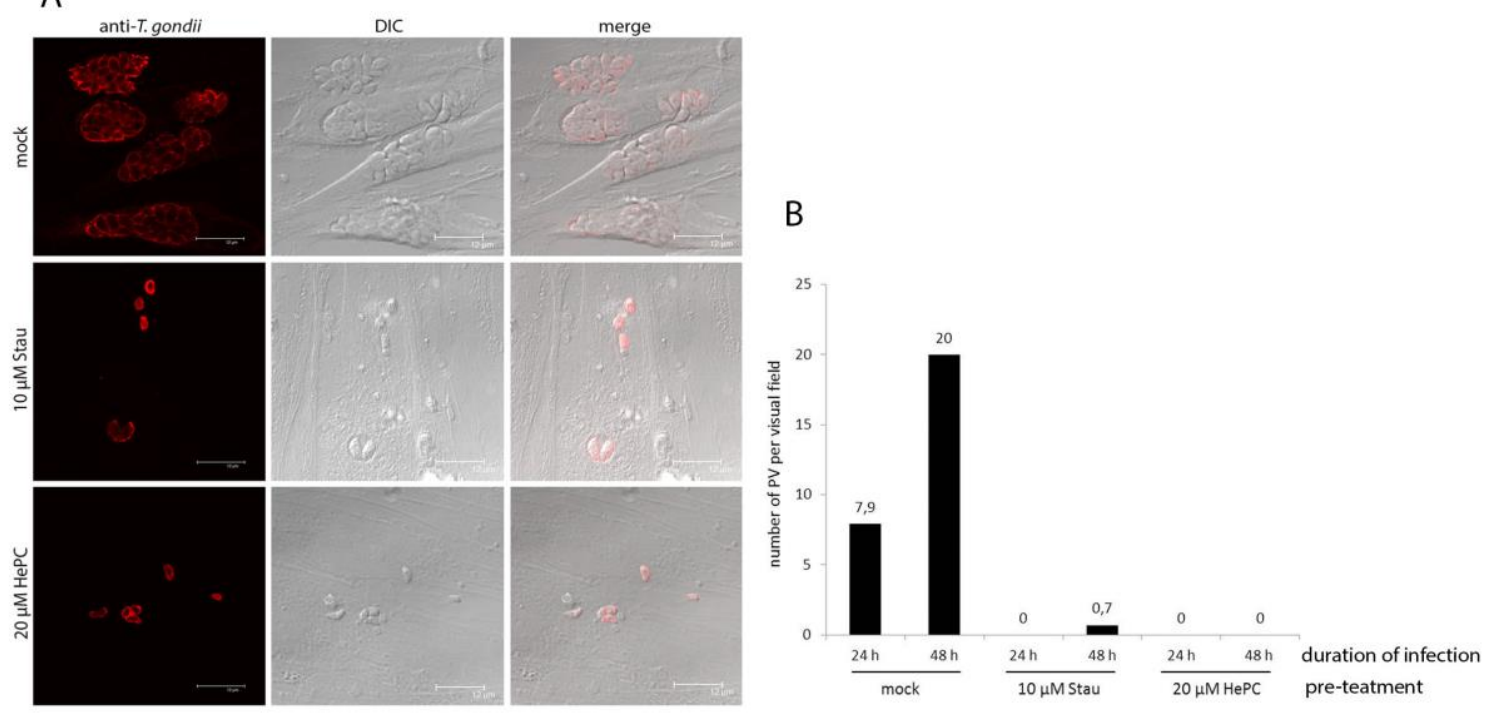

Figure 3.6. Pre-treatment of extracellular parasites with pro-apoptotic stimuli abrogate the ability of T. gondii to invade HFF cells. Extracellular parasites were pre-treated with $10 \mu \mathrm{M}$ staurosporine (stau) or $20 \mu \mathrm{M}$ miltefosine (HePC) or were mock-treated for $48 \mathrm{~h}$ prior to inoculation of confluent HFF cells. After 24 and $48 \mathrm{~h}$ of infection, cells were fixed and parasites were stained with parasite-specific antibodies. A, Images are representative of HFF cells that were inoculated for $48 \mathrm{~h}$ with parasites which had been treated with staurosporine and miltefosine as obtained by confocal laser scanning microscopy. DIC, differential interference contrast. B, Numbers of parasitophorous vacuoles (PVs) were determined from 10 randomly selected visual fields from each sample. Bars represent the average numbers of PV per visual field. Data is from 1 experiment.

\subsection{Physiological significance of apoptosis-like cell death in $T$. gondii}

There are an increasing number of reports on the existence of apoptosis-like cell death in single-celled organisms. Putative benefits of a self-destruction ability for protozoan parasites have been proposed to be the regulation of parasite densities, the evasion of immune responses of the host, the regulation of differentiation processes in parasites, and a counter mechanism under stress conditions in order to ensure perpetuation of infection (Al-Olayan et al., 2002; Ameisen et al., 1995; Bruchhaus et al., 2007; Duszenko et al., 2006; van Zandbergen et al., 2006; van Zandbergen et al., 2010).

In this study, we performed experiments to unravel the physiologic relevance of an apoptosis-like cell death in T. gondii. 


\subsubsection{TUNEL-positive parasites were observed during chronic infection with $T$. gondii, but not during acute infection}

The tachyzoite is the rapidly replicating stage of $T$. gondii and contributes to acute inflammation during T. gondii infection (Dubey et al., 1998; Kim and Weiss, 2004; Laliberte and Carruthers, 2008). A chronic infection of T. gondii is characterised by the presence of bradyzoites, a slow growing stage, within tissue cysts. The cysts contain hundreds or more bradyzoites and may persist for years without harming the host (Dubey et al., 1998; Pavesio et al., 1992).

To evaluate the appearance of an apoptosis-like cell death during the natural life cycle of $T$. gondii, mice (strain NMRI) were inoculated by defined amounts of parasites i.p. and the intraperitoneal fluid was isolated at 7, 10, and 12 days post infection. After fixing and permeabilising the intraperitoneal cells, the parasites were stained using antibodies against $T$. gondii and subsequently were stained by TUNEL assay. The results indicated that TUNEL-positive parasites were not prevalent during acute intraperitoneal inflammation at 7, 10 and 12 days post infection (Figure 3.7A). It suggests that apoptosislike cell death did not contribute to the regulation of tachyzoite density during acute infection in the peritoneal cavity of infected mice.

We furthermore tested whether apoptosis-like cell death occurs during chronic infection. For this purpose, NMRI mice were infected intraperitoneally by $T$. gondii tissue cysts (kindly provided by Dirk Schlüter, Magdeburg). After 30 weeks, brains from Toxoplasma-infected NMRI mice were isolated and homogenised by glass beads. The homogenised solution was then spread onto glass slides and fixed with methanol. Staining with antibodies recognizing bradyzoites of $T$. gondii within the brain cysts and concomitant TUNEL assays showed the appearance of TUNEL-positive bradyzoites within tissue cysts. Bradyzoites with TUNEL-positive feature were diffusely distributed within the tissue cysts (Figure 3.7B); no particular localisation, for example along the line of the cyts wall, was detected. Importantly, we could not detect the presence of TUNEL-positive parasites in young tissue cysts (consisting of only few bradyzoites). Thus, the results indicated that $T$. gondii employs an apoptosis-like cell death during chronic infection; it possibly depends on the age of the tissue cysts. 
A

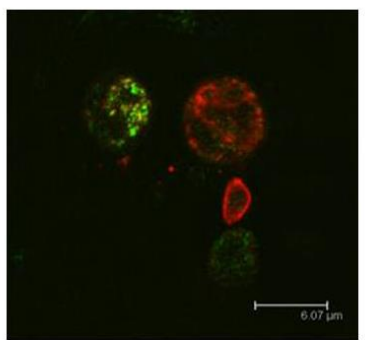

7 days p.i

B

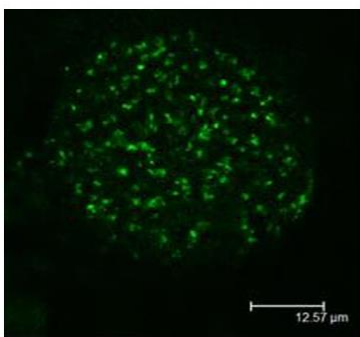

TUNEL

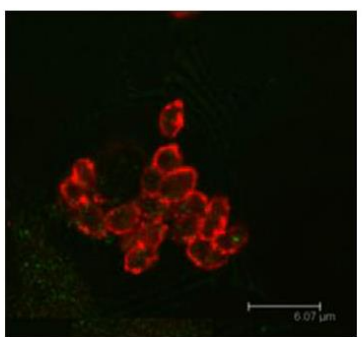

10 days $p . i$

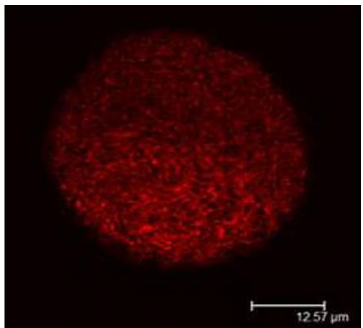

$\mathrm{DC} 11 \mathrm{Ab}$

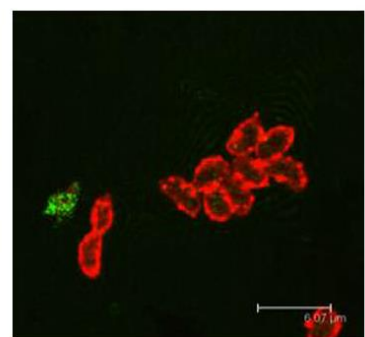

12 days p.i
anti-T. gondii

TUNEL

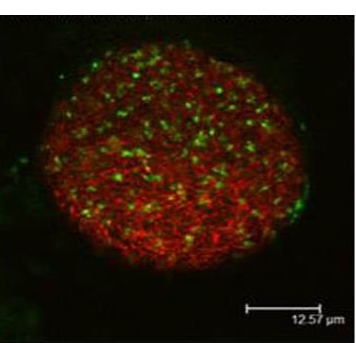

merge

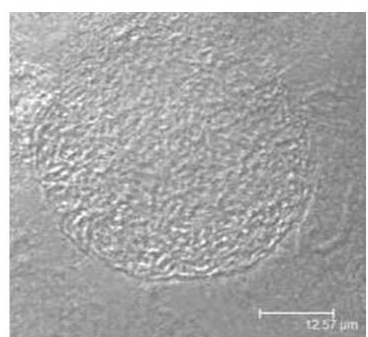

DIC

Figure 3.7. TUNEL-positive bradyzoites were detected during chronic infection of T. gondii. Mice were intraperitoneally inoculated with a defined amount of tachyzoites (for establishment of acute infection) or with a defined number of cysts (for development of chronic infection). A, At 7, 10, and 12 days post inoculation (p.i) with tachyzoites, intraperitoneal fluid was collected and then labelled using polyclonal $T$. gondii-specific antibodies and TUNEL reaction mix. B, Brains from chronically infected mice $(\sim 30$ weeks p.i) were isolated, homogenised and then stained using DC11 antibodies (bradyzoite-specific labelling) and TUNEL reaction mix. TUNEL-positive parasites (green labelling) were not observed during acute inflammation (A) but were recognised during chronic infection (B). 


\subsubsection{An apoptosis-like cell death occurred after treatment of tachyzoites with chemotherapeutic drugs commonly used against toxoplasmosis}

To determine whether $T$. gondii would undergo an apoptosis-like cell death under drug treatment, confluent HFF cells were inoculated with tachyzoites and were then treated with common drugs to treat toxoplasmosis. Parasites were labelled with parasite-specific antibodies and were subjected to TUNEL assay, and were analysed by confocal laser scanning microscopy.

The results showed a significant reduction of parasite replication following treatments of $T$. gondii with anisomycin, atovaquone or pyrimethamine as compared to the control treatment $(\mathrm{p}<0.01$; Figure 3.8A). At $48 \mathrm{~h}$ post treatment, numbers of parasites per PV decreased from $\sim 8$ in untreated parasites to $\sim 2-4$ in treated parasites. Because of its delayed parasiticidal effect (Fichera et al., 1995; Pfefferkorn et al., 1992), clindamycin effectively inhibited parasite replication only during the second but not the first replication cycle (Figure 3.8A; p <0.01). Remarkably, the number of TUNEL-positive extracellular parasites increased after treatment with all chemotherapeutics for $48 \mathrm{~h}$ as compared to the control with the strongest increase being induced using atovaquone $(\mathrm{p}<0.05)$ (Figure 3.8B; Figure 3.8C, arrowheads). Most importantly, a significant number of intracellular parasites which were strongly TUNEL-positive were also recognised during the second cycle of clindamycin treatment (Figure 3.8D; p<0.01). TUNEL-positive intracellular parasites following clindamycin treatment showed an abnormal morphology (Figure 3.8C, arrows). This data indicates that an apoptosis-like cell death can be triggered in extracellular and intracellular parasites undergoing drug treatment. 
A

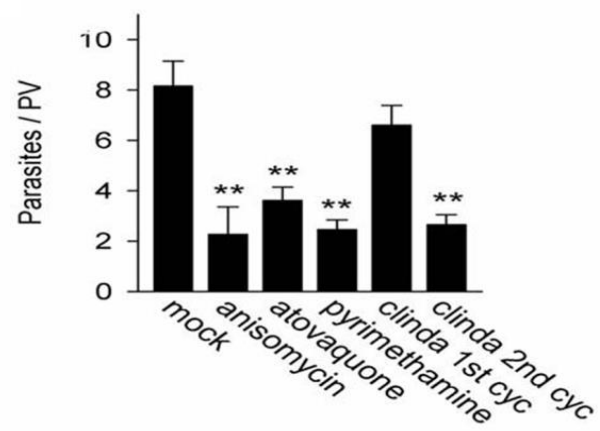

B

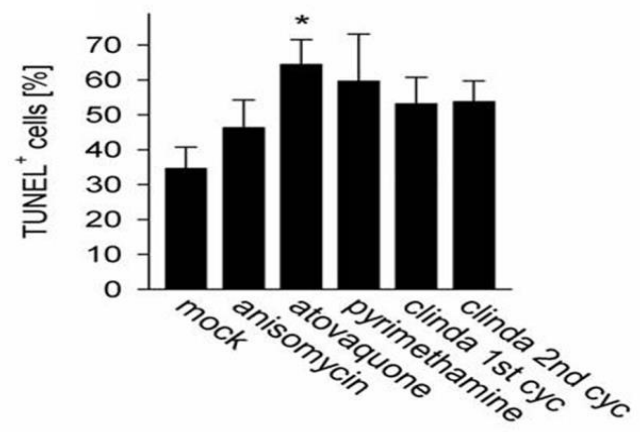

$\mathrm{C}$
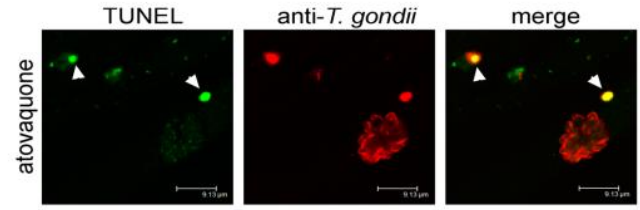

DIC

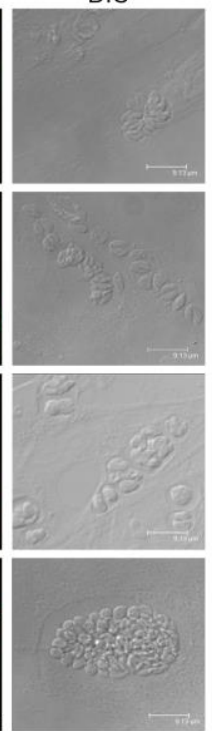

$\mathrm{D}$
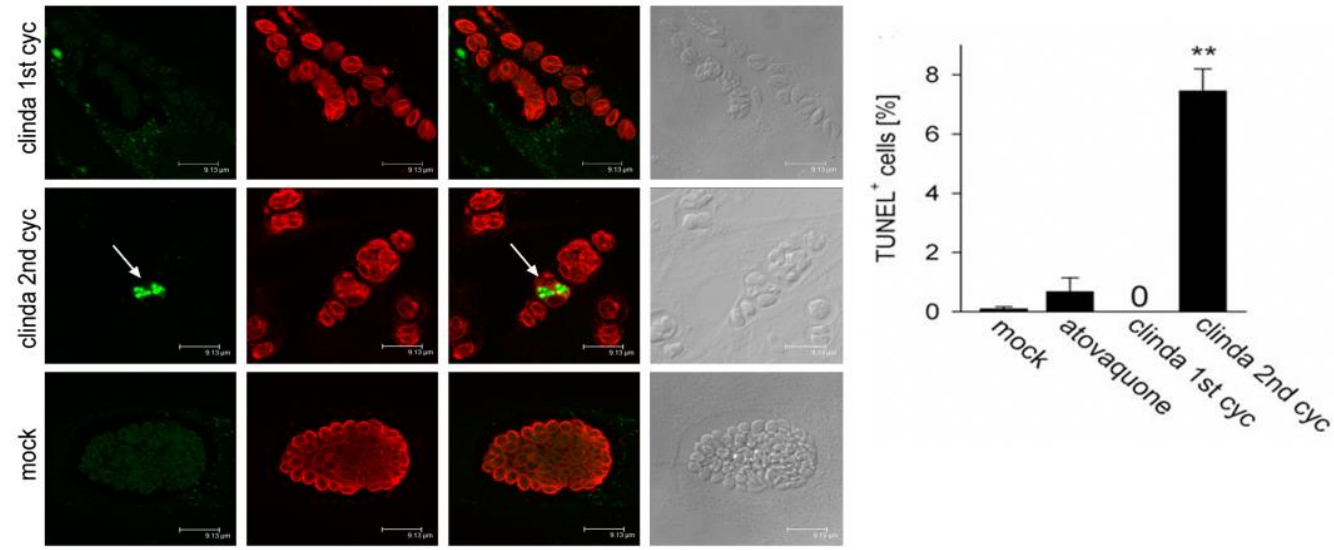

Figure 3.8. Induction of an apoptosis-like cell death in extracellular and intracellular $T$. gondii under drug treatments. Parasite-infected HFF cells were treated with $500 \mathrm{nM}$ anisomycin, $100 \mathrm{nM}$ atovaquone, $1 \mu \mathrm{M}$ pyrimethamine or $500 \mathrm{nM}$ clindamycin (clinda; for two consecutive cycles of parasite replication) or were mock-treated. After $48 \mathrm{~h}$ of treatment, parasites were stained using parasite-specific antibodies (red fluorescence) and DNA strand breaks were detected by in situ TUNEL assay (green fluorescence). A, Parasite replication was determined by calculating the average number of parasites per parasitophorous vacuole (PV). Bars represent means \pm S.E.M. from three independent experiments. B,D, TUNEL-positive extracellular (B) and intracellular (D) parasites. Bars represent means \pm S.E.M. from three independent experiments. C, Representative images of cells treated with the indicated drugs for $48 \mathrm{~h}$ were obtained by confocal laser scanning microscopy. TUNEL-positive extracellular parasites are marked by arrowheads (atovaquone), TUNEL-positive intracellular parasites are marked by arrows (second cycle of clindamycin treatment). DIC, differential interference contrast; $* p<0.05 ; * * p<0.01$. 


\subsection{Molecules which may be responsible for the regulation of apoptosis-like cell} death in T. gondii

Since molecules which might regulate or execute apoptosis-like cell death in $T$. gondii are yet unknown, experiments were conducted in order to unravel possible cell death pathways in $T$. gondii.

\subsubsection{Up-regulation of cell death-associated genes following treatment of $T$. gondii with pro-apoptotic stimuli}

Apoptosis is a highly complex and sophisticated mechanism, involving numerous components of the cell death machinery (Elmore, 2007; Vaux and Strasser, 1996). In order to identify putative cell death-associated molecules in $T$. gondii, a text-based in silico analysis of the $T$. gondii genome database (www.toxodb.org) was conducted. Seventeen cell death-associated genes were identified from the database, including a putative endonuclease G (TgME49_008710), a putative apoptosis inducing factor (AIF; TgME49_007620), a putative metacaspase (TgME49_006490), putative Bax inhibitors (hypothetical proteins; TgME49_049770, TgME49_109560 and TgME49_003030), putative calpains (TgME49_093820 and TgME49_093830), beclin-1 (TgME49_021360), putative ICE-like protease (TgME49_078970), putative ELMO-like protein (TgME49_053000), putative programmed cell death proteins (TgME49_094420 and TgME49_105490), a putative defender against cell death (DAD; TgME49_105870), a putative apoptosis-regulating basic protein (TgME49_035880), and other hypothetical proteins (TgME49_035560 and TgME49_107780) (Table 3.1).

Table 3.1. Toxoplasma gondii cell death-associated genes

\begin{tabular}{lll}
\hline Gene ID & Name & Annotation \\
\hline TgME49_008710 & $\begin{array}{l}\text { DNA/RNA non-specific } \\
\text { endonuclease domain-containing } \\
\text { protein }\end{array}$ & $\begin{array}{l}\text { nucleic acid } \\
\text { binding/endonuclease activity/ } \\
\text { hydrolase activity/metal ion } \\
\text { binding }\end{array}$ \\
TgME49_007620 & $\begin{array}{l}\text { Pyridine nucleotide-disulphide } \\
\text { oxidoreductase domain- } \\
\text { containing protein }\end{array}$ & $\begin{array}{l}\text { oxidation reduction/electron } \\
\text { carrier activity/FAD } \\
\text { binding/electron transport }\end{array}$
\end{tabular}


TgME49_078970 ICE-like protease (caspase) p20 domain-containing protein

TgME49_053000 ELMO/CED12 family domaincontaining protein

TgME49_094420 Programmed cell death protein, putative

TgME49_093820 Calpain family cysteine protease domain-containing protein

TgME49_093830 Calpain, putative

TgME49_021360 Beclin-1, putative

TgME49_049770 Hypothetical protein, conserved

TgME49_109560 Hypothetical protein, conserved

TgME49_003030 Hypothetical protein

TgME49_105490 Programmed cell death protein, putative

TgME49_105870 Dolichyl-

diphosphooligosaccharideprotein glycotransferase, putative

TgME49_035560 Hypothetical protein

TgME49_107780 Hypothetical protein proteolysis/cysteine-type endopeptidase activity/caspase activity

phagocytosis

no further information

proteolysis/calcium-dependent cysteine-type endopeptidase activity

metabolic

process/methyltransferase activity

autophagy

Homologue of TgVEG 'Fas apoptotic inhibitory molecule, putative'

Homologue of TgVEG 'Fas apoptotic inhibitory molecule, putative'

Homologue of TgVEG 'Fas apoptotic inhibitory molecule, putative'

no further information

Homologue of TgGT1, TgVEG 'defender against cell death, putative'

activation of caspase activity/protein binding/induction of apoptosis

proteolysis/calcium-dependent cysteine-type endopeptidase activity 


\begin{tabular}{lll} 
TgME49_035880 & $\begin{array}{l}\text { Apoptosis-regulating basic } \\
\text { protein, putative }\end{array}$ & no information \\
TgME49_006490 & $\begin{array}{l}\text { Metacaspase-1 precursor, } \\
\text { putative }\end{array}$ & $\begin{array}{l}\text { proteolysis/cysteine-type } \\
\text { endopeptidase activity/caspase } \\
\text { activity }\end{array}$ \\
\hline
\end{tabular}

To examine the expression levels of the selected genes upon induction of apoptosis, total RNA from staurosporine- and miltefosine-induced parasites as well as from control parasites was extracted followed by cDNA synthesis. Using a set of primer pairs for the selected genes (Chapter 2, section 2.1.7, Table 2.5), the expression level of these genes was measured through quantitative real-time reverse transcriptase PCR. These analyses revealed that transcripts of all of the selected genes were detected in freshly isolated parasites and that their expression did not change until $48 \mathrm{~h}$ of mock treatment (Figure 3.9A,B).

Following staurosporine treatment, several mRNAs of the seventeen selected genes increased in a time-dependent fashion (Figure 3.9A). The strongest up-regulated transcript was PDCD2 (TgME49_105490), which increased 100-fold at $48 \mathrm{~h}$ post staurosporine treatment. A strong up-regulation of mRNAs at $48 \mathrm{~h}$ after staurosporine treatment was also detected for putative ELMO (TgME49_053000) and DNA/RNA endonuclease (TgME49_008710) transcripts which both increased more than 20-fold, a putative Fas apoptotic inhibitory molecule (hypothetical protein; TgME49_049770) which increased 18-fold and two calpain-like proteins (TgME49_093820 and TgME49_093830) which increased more than 10-fold. Treatment with miltefosine also strongly up-regulated the transcript of PDCD2 (more than 40-fold at $48 \mathrm{~h}$ ) while other transcripts were only moderately up-regulated or even not regulated (Figure 3.9B).

Together, the data suggested that components of ancient PCD machinery may exist in $T$. gondii, the expression of which is differentially regulated upon induction of cell death. 


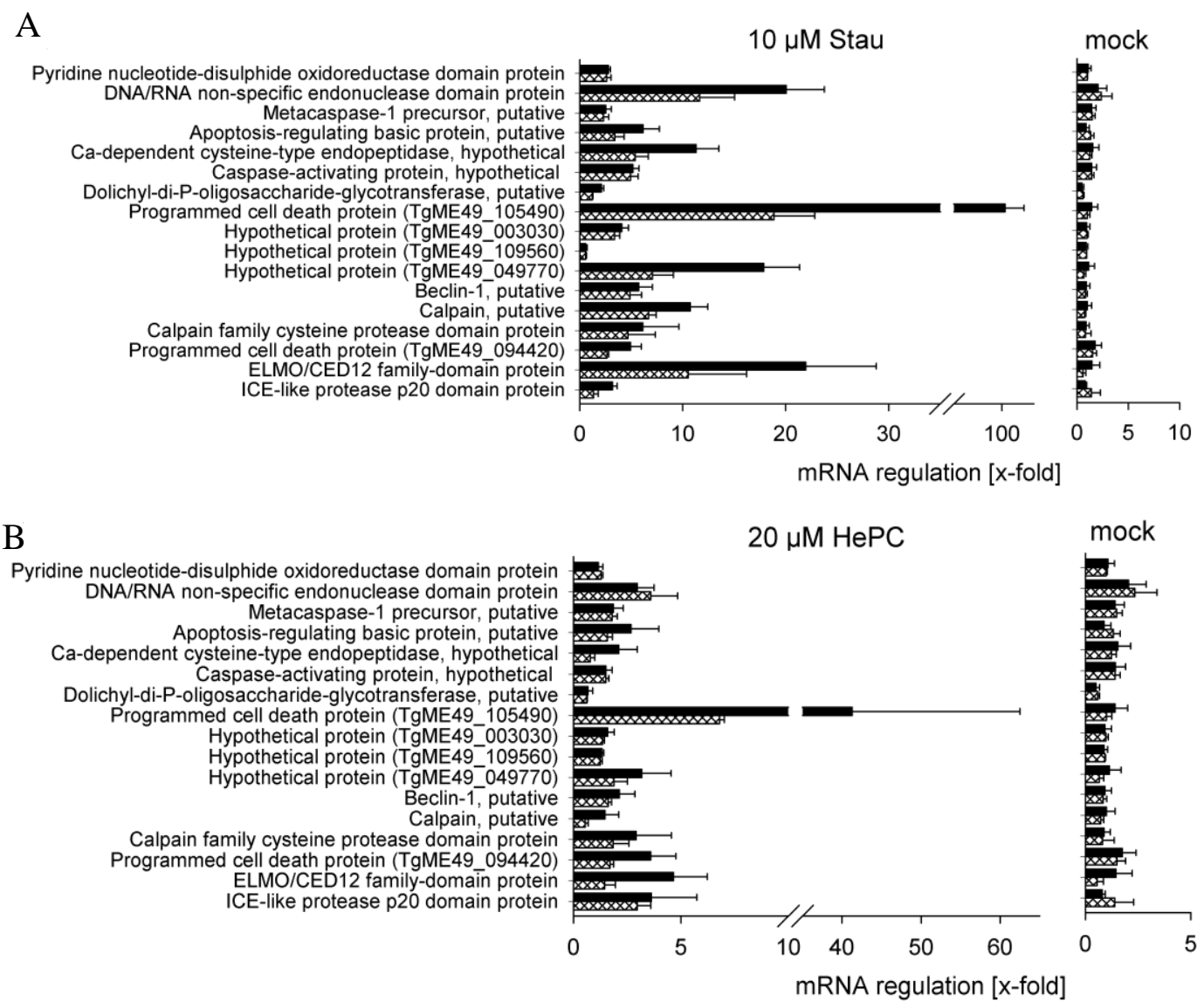

Figure 3.9. Transcript levels of $T$. gondii putative PCD-associated genes after treatment of parasites with proapoptotic stimuli. Parasites were treated with $10 \mu \mathrm{M}$ staurosporine (stau) or $20 \mu \mathrm{M}$ miltefosine (HePC), or were mock-treated for $24 \mathrm{~h}$ (cross-hatched bars) and $48 \mathrm{~h}$ (black bars). After RNA isolation and reverse transcription, cDNA was amplified by quantitative real-time PCR using specific primer pairs for the indicated cDNAs or actin of $T$. gondii. Results are described as the mean ratios $(x-$ fold $) \pm$ S.E.M. $(n=3)$ of the mRNA after treatments as compared to freshly isolated parasites and are normalised to actin.

Considering that $T$. gondii do not express genuine caspases, we further focused in our study on T. gondii Bax inhibitors (TgBIs) and Endonuclease G (TgEndoG). Both proteins are considered to play roles in ancient PCD pathways.

\subsubsection{Toxoplasma gondii Bax inhibitors (TgBIs)}

\subsubsection{Bioinformatic analysis}

In silico analysis of the T. gondii genomic database demonstrated the presence of three putative Bax inhibitor genes, named T. gondii Bax inhibitor-1, -2 , and -3 (TgBI-1, 
TgBI-2 and TgBI-3). Amino acid sequence analysis of TgBI-1, TgBI-2 and TgBI-3 showed 33-38 \% identity with human BI-1. Putative TgBI-1, TgBI-2 and TgBI-3 contain 6-9 transmembrane (TM) domains and have a predicted molecular weight of 37,974 Da, 29,322 Da and 37,345 Da, respectively (Table 3.2). Conserved C-terminal charged residues EAVNKK in human Golgi Anti-Apoptotic Protein (hGAAP) or EKDKKEKK in human Bax Inhibitor 1 (hBI-1) which are important for their anti-apoptotic, cell adhesion and calcium regulatory functions (Carrara et al., 2012) could not be identified in TgBIs; TgBIs showed no conserved domains that could be used to predict their molecular function.

Table 3.2. Characteristics of TgBIs

\begin{tabular}{|c|c|c|c|}
\hline Name/ID & Amino acids & Tool & TM domain ${ }^{1}$ \\
\hline TgBI-1 & 339 & TMHMM2.0 & 9 \\
\hline \multirow[t]{6}{*}{ TgME49_049770 } & & SOSUI & 9 \\
\hline & & Pfam & 9 \\
\hline & & MEMSAT_SVM & 7 \\
\hline & & MEMSAT3 & 6 \\
\hline & & SCAMPI & 9 \\
\hline & & TopPred 0.01 & 9 \\
\hline TgBI-2 & 265 & TMHMM2.0 & 7 \\
\hline \multirow{6}{*}{ TgME49_109560 } & & SOSUI & 7 \\
\hline & & Pfam & 7 \\
\hline & & MEMSAT_SVM & 7 \\
\hline & & MEMSAT3 & 6 \\
\hline & & SCAMPI & 7 \\
\hline & & TopPred 0.01 & 7 \\
\hline TgBI-3 & 342 & TMHМM2.0 & 7 \\
\hline \multirow[t]{6}{*}{ TgME49_003030 } & & SOSUI & 7 \\
\hline & & Pfam & 7 \\
\hline & & MEMSAT_SVM & 7 \\
\hline & & MEMSAT3 & 7 \\
\hline & & SCAMPI & 7 \\
\hline & & TopPred 0.01 & 7 \\
\hline
\end{tabular}

\footnotetext{
${ }^{1}$ The membrane topology of TgBIs was predicted using different tools as indicated above. Data showed different predicted numbers of transmembrane (TM) domains of TgBIs.
} 


\subsubsection{Immunofluorescence assay of $T$. gondii Bax inhibitors demonstrated distinct}

\section{localisations of the proteins}

Coding sequences encoding TgBI-1, -2 and -3 (kindly provided by Dominique Soldati-Favre, Geneva) have been subcloned into the mammalian expression vectors pCMV-SC-NF or pCMV-SC-CF. These vectors contain an N- or C-terminal FLAG-tag that allows the detection of recombinant protein. To unravel possible functions of $T$. gondii BI proteins in the regulation of apoptosis, TgBIs coding genes were heterologously expressed in HeLa cells. HeLa cells are widely used to investigate apoptosis mechanisms, therefore we used HeLa cells in these experiments as a model to analyse the TgBIs function. Stable transfection of the genes encoding TgBI-1, TgBI-2 and TgBI-3 into HeLa cells was confirmed by immunofluorescence microscopy.

Detection of expression and localisation of the recombinant TgBIs was performed by immunofluorescence assay (Figure 3.10). To this end, wildtype (WT) HeLa cells and TgBIs-expressing HeLa cells were seeded in a 24-well plate containing cover slips. After $24 \mathrm{~h}$, cells were fixed and stained with anti-FLAG antibodies and were then visualised using a confocal laser scanning microscopy. Interestingly, the result showed clear differences in the localisations of the different TgBIs; TgBI-1 had a perinuclear localisation, TgBI-2 had a predominant cell surface expression and TgBI-3 had both a perinuclear and cell surface localisation. 

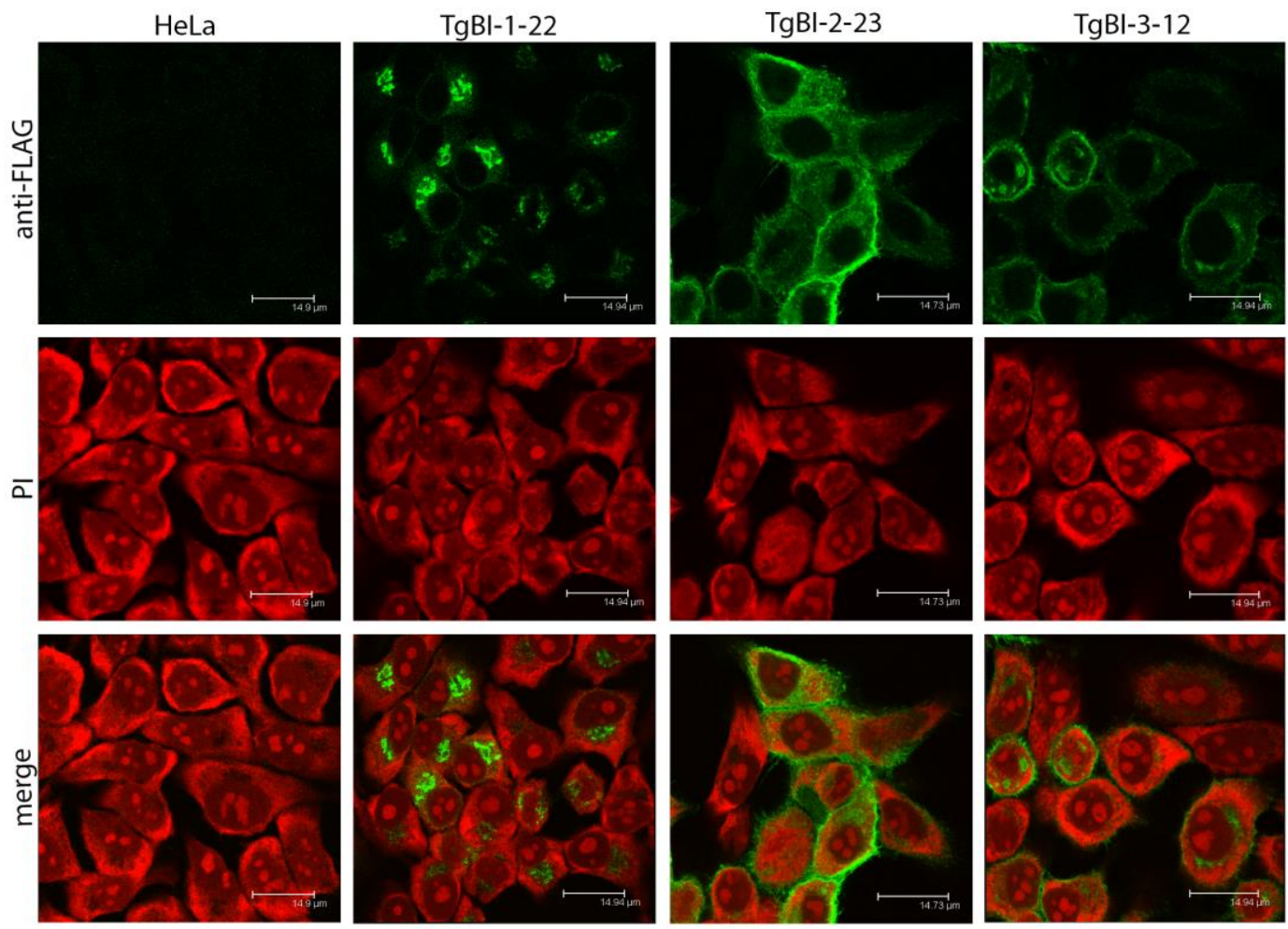

Figure 3.10. Expression and localisation of TgBIs in HeLa cells was detected by immunofluorescence microscopy. Detection of recombinant proteins of TgBI-1 (clone 22), TgBI-2 (clone 23) and TgBI-3 (clone 12) was performed using anti-FLAG and fluorescently labelled secondary antibodies (green fluorescence). Propidium iodide (PI; red fluorescence) is a DNA-binding dye. It was used to stain the nuclei and to visualise the whole cell population. Images were obtained by confocal laser scanning microscopy.

\subsubsection{TgBIs showed a non-mitochondrial localisation}

Bax inhibitor-1 is an evolutionary conserved protein that mainly localises to ER membranes, but can also be detected in mitochondria (Chae et al., 2004; Xu and Reed, 1998). Moreover, Leishmania Bax inhibitor has been found in mitochondria (Antonio Jiménez-Ruiz, Madrid; personal communication). To determine whether heterologously expressed TgBIs also have a mitochondrial localisation, Mitotracker (a mitochondria probe) co-staining was used in our study.

To this end, Hela WT and TgBIs-expressing HeLa cells were seeded on cover slips in a 24-well plate. After $24 \mathrm{~h}$, cells were incubated with Mitotracker dye and then immunofluorescently labelled using an anti-FLAG antibody. TgBIs (either TgBI-1, TgBI- 
2, or TgBI-3) did not colocalise with characteristic mitochondria (Figure 3.11), indicating that TgBIs have a non-mitochondrial localisation.

Detection of the protein localisation is important because the localisation of a protein and its physiological function are closely related (Bolte and Cordelieres, 2006). Therefore, an approach using well-characterised organelle markers is required to precisely visualise the location of TgBIs.

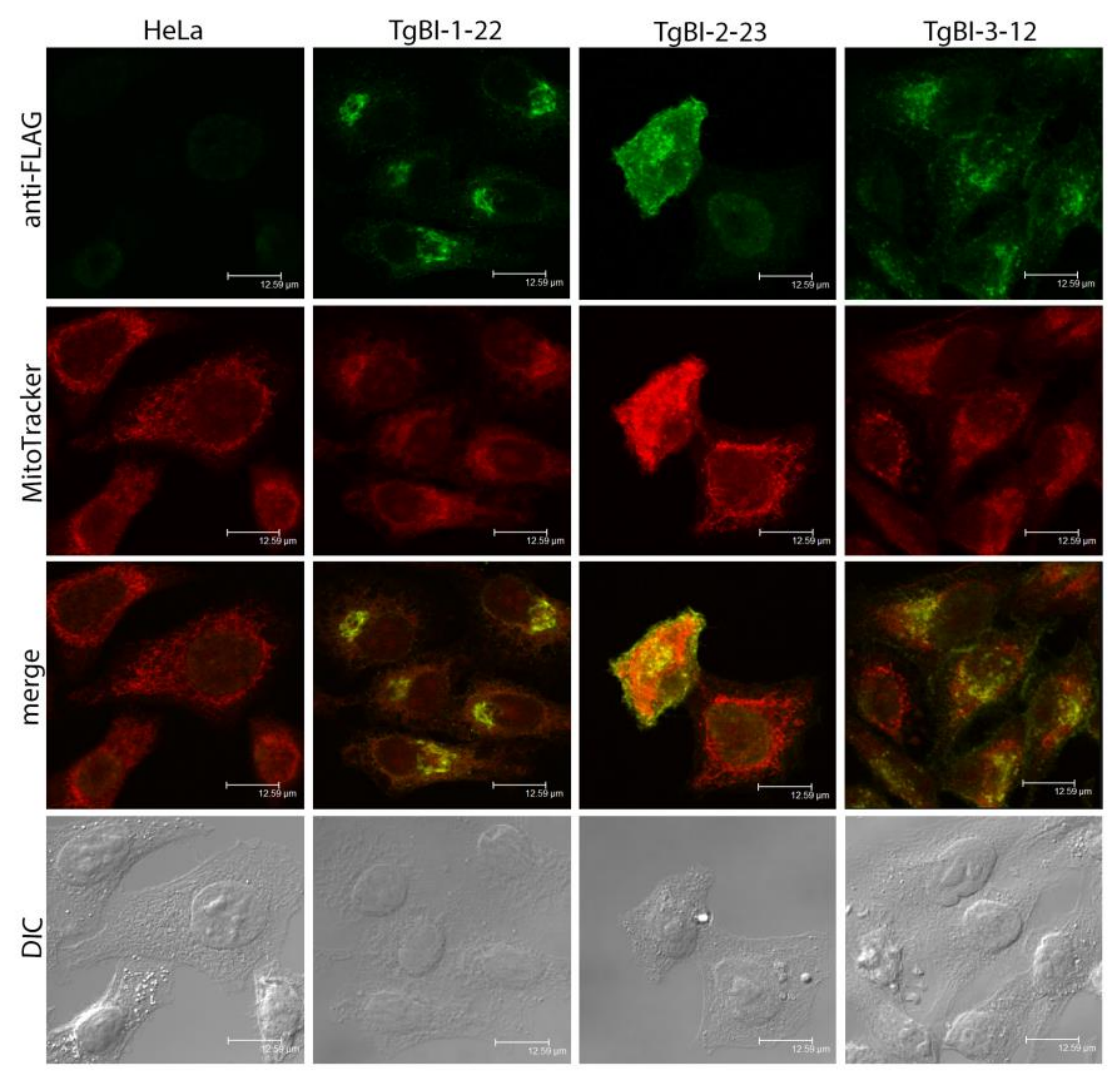

Figure 3.11. Heterologous expression of TgBIs in HeLa cells showed a non-mitochondrial localisation as detected by MitoTracker and anti-FLAG co-staining. HeLa cells transfected with TgBI-1 (clone 22), TgBI-2 (clone 23) or with TgBI-3 (clone 12) or HeLa WT cells were grown on round cover slips in a 24-well plate. After staining with MitoTracker ${ }^{\circledR}$ Orange CM-H2TMRos, the cells were fixed and were then stained with anti-FLAG antibodies. Visualisation of the cells was done using confocal laser scanning microscopy. Images of both labellings were sequentially recorded in order to avoid a crosstalk between MitoTracker (red fluorescence) and anti-FLAG-tag labelling (green fluorescence). DIC, differential interference contrast. 


\subsubsection{Redistribution of TgBIs upon cell death induction}

To evaluate the effect of cell death inducers on the cellular distribution of TgBIs, we incubated Hela WT, TgBI-2- and TgBI-3-transfected HeLa cells (stable transfectants of TgBI-1 have not yet been obtained when we performed this experiment) in the presence of staurosporine or brefeldin A (breA). Staurosporine has been widely used to induce apoptosis in various cell types, but the mechanism to initiate apoptosis remains largely undefined. Staurosporine has been described to induce apoptosis in melanoma cells by both caspase-dependent and caspase-independent pathways (Zhang et al., 2004). Brefeldin A is a fungal antibacterial agents that blocks transport of newly synthesised proteins from ER to the Golgi apparatus, but not the retrograde transport of proteins back to the ER (Lippincott-Schwartz et al., 1989; Miller et al., 1992). After treatments with staurosporine and brefeldin A for 24 and $48 \mathrm{~h}$, the HeLa cells and TgBIs-expressing HeLa cells were fixed, then stained with anti-FLAG antibodies and analysed using confocal laser scanning microscopy.

Following staurosporine treatment, $\mathrm{TgBI}-2$ protein maintained its predominant cell surface localisation (Figure 3.12). However, the protein redistributed to the cytosol and lost the cell surface expression after brefeldin A treatment. On the other hand, TgBI-3 protein lost its perinuclear and cell surface expression during both treatments with different characteristics; staurosporine led to a rather diffuse localisation of TgBI-3 within the cytosol while brefeldin A treatment caused a punctate pattern of the protein within the cytosol. The results suggested the existence of distinct mechanisms of Bax inhibitor proteins to respond to different stimuli of cell death. 


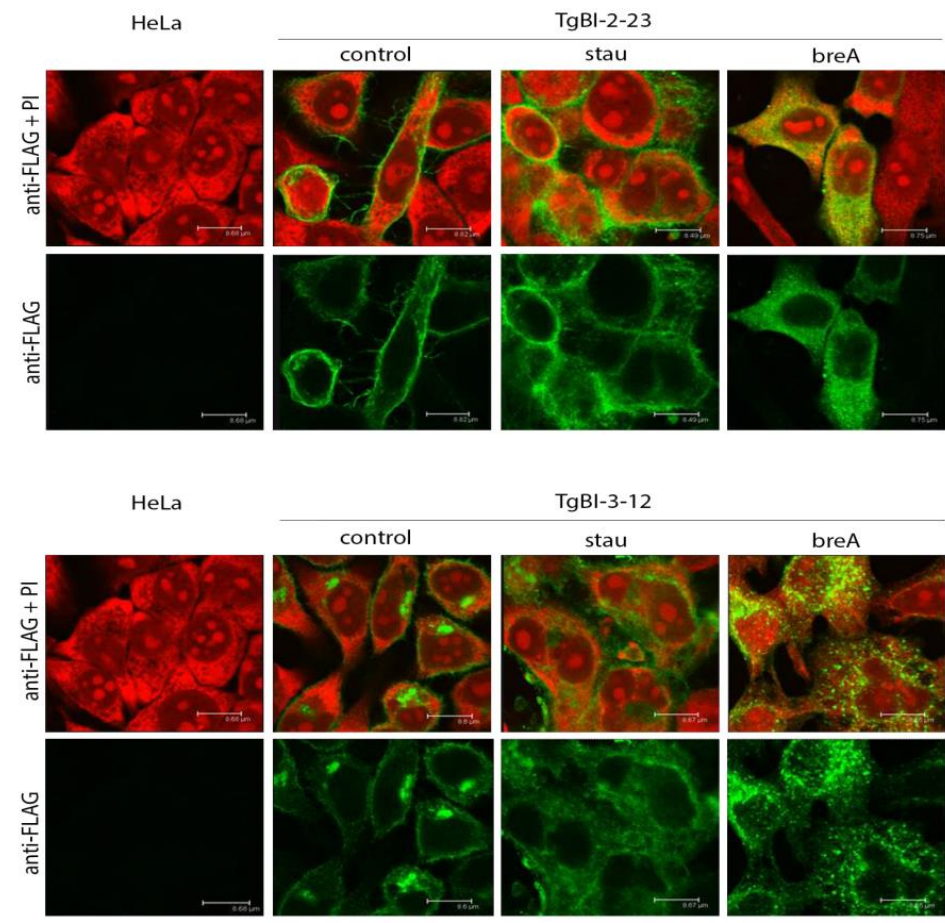

Figure 3.12. Redistribution of TgBI-2 and TgBI-3 following staurosporine or brefeldin A treatment. HeLa cells transfected with TgBI-2 (clone 23) or with TgBI-3 (clone 12) or HeLa WT cells were grown on round cover slips in a 24 -well plate and were then treated with $0.3 \mu \mathrm{M}$ staurosporine (stau) or $10 \mu \mathrm{M}$ brefeldin A (breA) or were left untreated (control). Images represent TgBI-2 and TgBI-3 localisation at $24 \mathrm{~h}$ post treatment. The upper panel shows representative images of TgBI-2-transfected HeLa cells and the lower panel those of TgBI-3-transfected HeLa cells. Images were recorded by confocal laser scanning microscopy after labelling of TgBIs with anti-FLAG antibodies (green fluorescence) and propidium iodide staining (red fluorescence). Note the different distributions of the proteins following treatment with pro-apoptotic stimuli.

\subsubsection{TgBIs in HeLa cells partially inhibited caspase $3 / 7$ activity following cell} death induction

Caspases are activated during apoptosis induction in mammalian cells. To determine whether caspases are activated in TgBIs-transfected HeLa cells upon cell death stimulation, we performed a caspase activity assay using a fluorescently labelled synthetic substrate, DEVD-AMC (a substrate of caspase 3 and 7). The substrate will be cleaved in the presence of active caspases and the released fluorochrome will fluoresce. The caspase activity was assessed by calculating the increase of fluorescence intensity in cellular lysates from 5 to $60 \mathrm{~min}$. 
To this end, HeLa WT and TgBIs-expressing HeLa cells were seeded and incubated for 24 and $48 \mathrm{~h}$ in cell culture medium containing staurosporine and brefeldin A. Total soluble proteins were collected by lysing the cells and centrifugation. Measurement of caspase 3/7 activity revealed an inhibitory effect of TgBI-1 as well as TgBI-2 in HeLa cells after treatment with staurosporine and of TgBI-2 after brefeldin A treatment (Figure 3.13). Additionally, TgBI-2 also showed a protective effect against cell death induction in HeLa cells following treatment with $5 \mu \mathrm{g} / \mathrm{ml}$ tunicamycin, an $\mathrm{N}$-glycosidic linkage blocker, at 24 and $48 \mathrm{~h}$ post treatment and following treatment with $2 \mu \mathrm{M}$ thapsigargin, an endoplasmic reticulum calcium pump inhibitor, at $48 \mathrm{~h}$ post treatment (data not shown). In contrast, heterologous expression of TgBI-3 in HeLa cells did not inhibit caspase activity following both staurosporine and brefeldin A treatments (Figure 3.13). The results suggested a conserved ability of TgBIs to partially inhibit apoptosis which is in mammals executed via a caspase cascade.
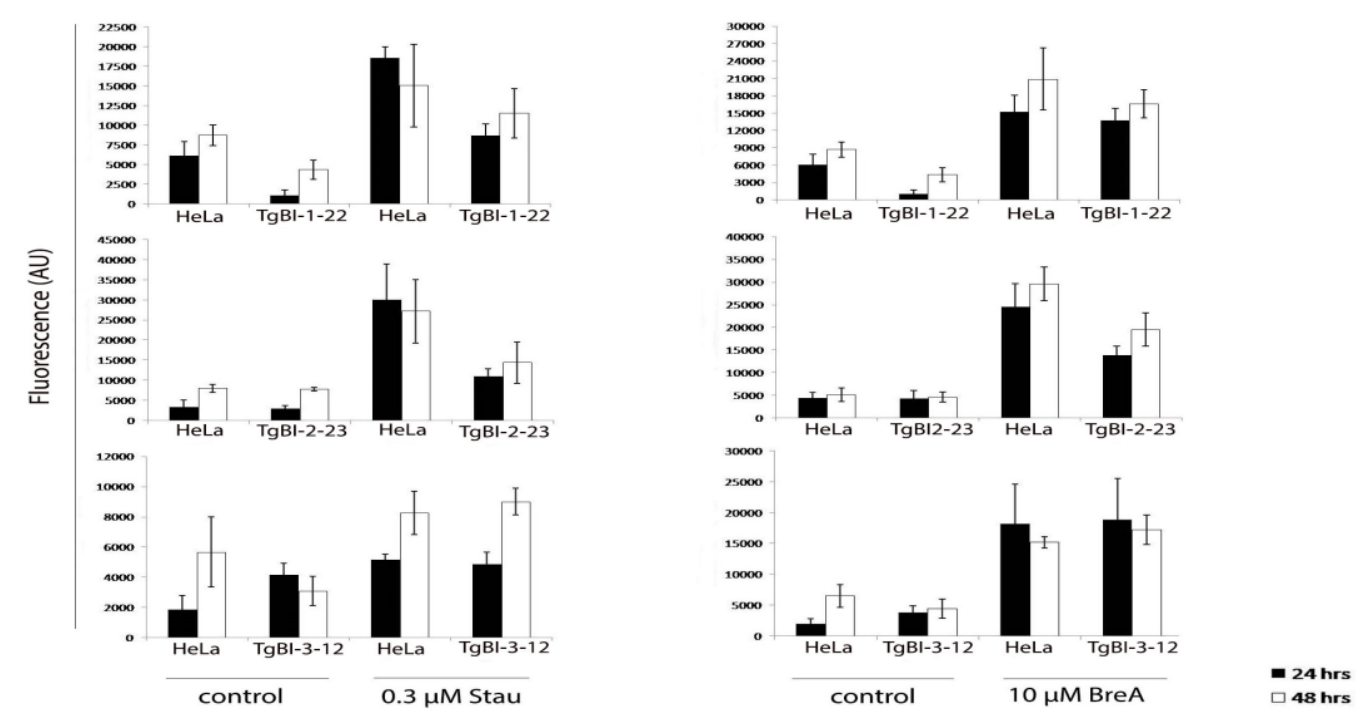

Figure 3.13. Measurement of caspase 3/7 activity partially revealed an inhibitory effect of TgBI-1 and TgBI2, but not TgBI-3, on apoptosis induction in HeLa cells after treatment with staurosporine and brefeldin A. HeLa WT and distinct clones of TgBIs-expressing HeLa cells (TgBI-1-22, TgBI-2-23 and TgBI-3-12) were cultivated in the presence of $0.3 \mu \mathrm{M}$ staurosporine (stau) or $10 \mu \mathrm{M}$ brefeldin A (BreA) of were left untreated (control). At 24 and 48 h, total soluble proteins were collected by lysing the cells. Caspase activity in cellular lysates was determined by measuring the fluorescence intensity after cleavage of a fluorochrome-conjugated synthetic substrate, DEVD-AMC. Bars represent means \pm S.E.M. from three experiments of TgBI-1 (upper panel), TgBI-2 (middle panel) and TgBI-3 (lower panel). Caspase activities after $24 \mathrm{~h}$ of treatment are depicted as black bars and those from $48 \mathrm{~h}$ post treatment are depicted as open bars. 


\subsubsection{TgBIs in HeLa cells reduced the induction of hypoploid DNA following pro- apoptotic treatments}

During apoptosis, DNA is fragmented and partially lost from the apoptotic nuclei due to the formation of apoptotic bodies, resulting in hypoploid nuclei (expressed as sub G0/G1 peak) (Krysko et al., 2008). To quantitatively measure apoptotic nuclei in TgBIsexpresssing HeLa cells, propidium iodide (a fluorescent dye that binds to nucleic acids) staining and flow cytometry have been performed.

For this purpose, HeLa and TgBIs-expressing HeLa cells were grown and treated with staurosporine or brefeldin A or were left untreated for 24 and $48 \mathrm{~h}$. To remove contaminating RNA, RNA was digested using RNase A prior to staining the nuclei using propidium iodide. Stained cells were then analysed by flow cytometry.

Heterologous expression of TgBI-1 led to a significant reduction in the appearance of cells with sub G0/G1 nuclei as compared to control HeLa WT cells at $24 \mathrm{~h}$ after treatment with brefeldin A (Figure 3.14B; p<0.01). Following staurosporine treatment, TgBI-1 showed a slight reduction of hypoploid DNA at $48 \mathrm{~h}$ post treatment. TgBI-2 exhibited a trend to reduce the induction of hypoploid DNA after treatment with both stimuli (Figure 3.14 A,C). TgBI-3 in HeLa cells showed a significant reduction of hypoploid DNA after treatment with both pro-apoptotic inducers at $24 \mathrm{~h}$ post treatment (Figure 3.14D; $\mathrm{p}<0.05$ ). At $48 \mathrm{~h}$ post treatment, we observed a significant decrease in the appearance of hypoploid DNA after staurosporine treatment $(\mathrm{p}<0.05)$ and a trend of TgBI3 to reduce brefeldin A-induced hypoploid DNA.

Collectively, these results indicated an evolutionary conserved protective effect of Bax inhibitors of $T$. gondii to reduce cell death induction in mammals and suggested a role of TgBIs in the regulation of apoptosis-like cell death in $T$. gondii. 
A

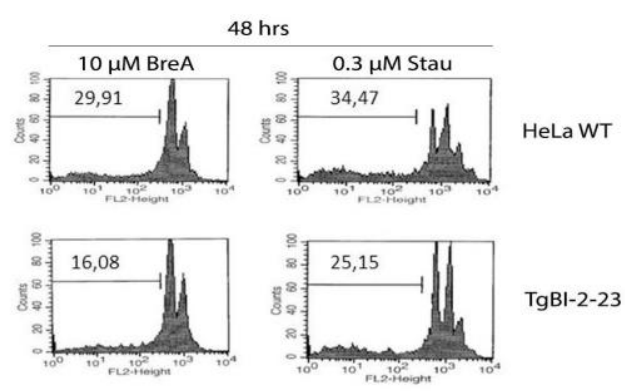

$\mathrm{C}$

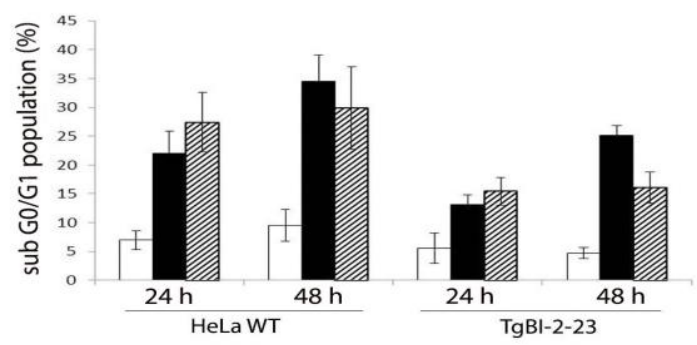

B

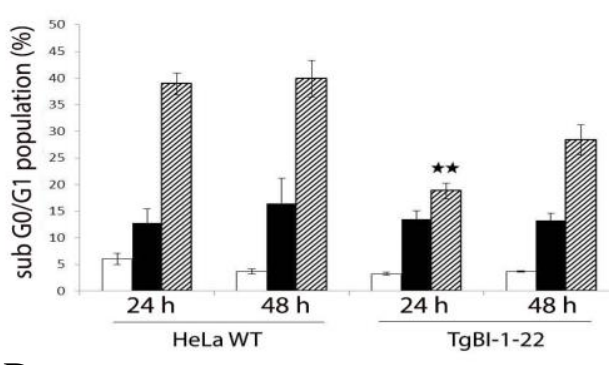

$\mathrm{D}$

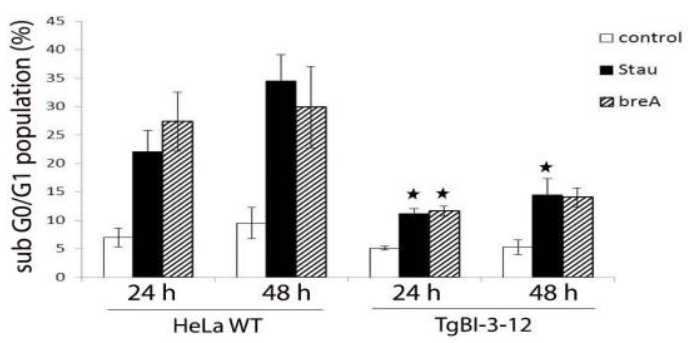

Figure 3.14. Inhibition of hypoploid nuclei by TgBIs upon cell death stimulation in HeLa cells. TgBIsexpressing HeLa cells (clones TgBI-1-22, TgBI-2-23 and TgBI-3-12) were treated with $0.3 \mu \mathrm{M}$ staurosporine (stau) or $10 \mu \mathrm{M}$ brefeldin A (breA) or were left untreated (control). A, Representative FACS histograms of TgBI-2-expressing HeLa cells are depicted. Numbers within the histogram are percentages of sub G0/G1 cells of TgBI-2 in comparison to HeLa WT at $48 \mathrm{~h}$ after treatment with cell death inducers. B, C, D, Bars represent means \pm S.E.M. $(n=3$ for TgBI-1, $n=4$ for TgBI-2 and TgBI-3) of sub G0/G1 nuclei of TgBI-1-, TgBI-2- and TgBI-3-expressing HeLa cells, respectively. Untreated controls are shown as open bars, cells treated with staurosporine are shown as filled bars and cells treated with brefeldin A are shown as hatched bars. $* * p<0.01 ; * p<0.05$.

\subsubsection{Toxoplasma gondii Endonuclease G (TgEndoG)}

\subsubsection{Bioinformatic analysis}

A candidate protein that might contribute to the execution of apoptosis-like cell death in $T$. gondii is Endonuclease G (TgEndoG). The corresponding gene was identified during our in silico analysis of the T. gondii genome database (see section 3.3.1; TgME49_008710). The predicted protein shows $31.5 \%, 38.1 \%, 36.1 \%$ and $35.3 \%$ identity with EndoG of Homo sapiens, Mus musculus, Bos taurus and S. cerevisiae, respectively. The putative TgEndoG consists of 894 amino acids and has a molecular weight of $96.2 \mathrm{kDa}$, much higher than the $\sim 30 \mathrm{kDa}$ of mammalian EndoG (van Loo et al., 2001 ) or the $\sim 60 \mathrm{kDa}$ of Leishmania EndoG (Rico et al., 2009). The active site of 
mammalian EndoG is characterised by a DRGH motif which is replaced by a SKGH motif in T. gondii (Figure 3.15, upper panel). Additionally, asparagine $(\mathrm{N})$, an important residue for divalent ion binding to support function of EndoG, is conserved in TgEndoG. The structure of TgEndoG was predicted using PyMOL Molecular Graphic System. The results showed that the structure of TgEndoG is compatible with an active Endonuclease $\mathrm{G}$ (Figure 3.15B) (collaboration with Antonio Jiménez-Ruiz, Universidad de Alcalá, Madrid).

A

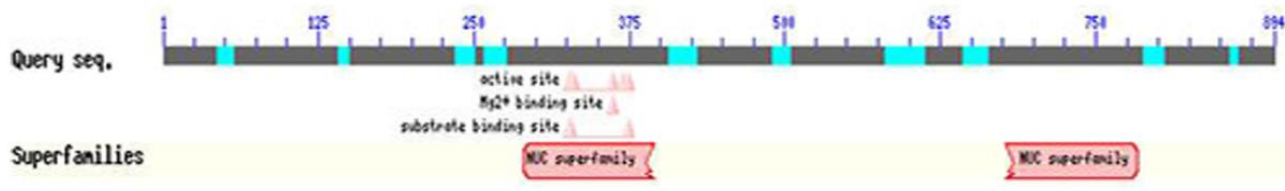

sp |Q14249|NUCG_HUMAN sp|P38447|NUCG_BOVIN sp|O08600|NUCG MOUSE SP|P08466/NUC1_YEAST tr|B 6 KP 68 |B 6 KP $\overline{6} 8$ TOXGO

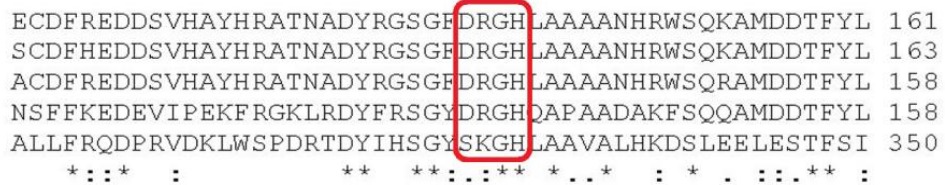

NSFFKEDEVIPEKF RGKLRDYFRSGYDRGHPAPAADAKFSQQAMDDTFYL 158

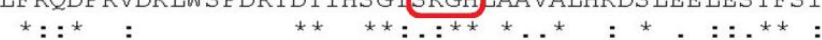

\section{B}

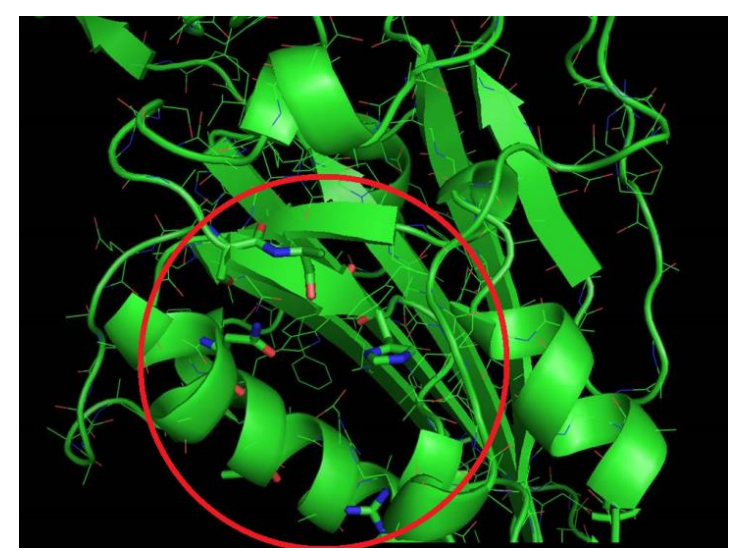

Figure 3.15. Alignment of EndoG sequences from different species and prediction of TgEndoG structure. A, TgEndoG consists of two non-specific endonuclease domains. The DRGH motif which is typical for EndoG of mammals and yeast and constitutes the active site domain, was replaced by SKGH in TgEndoG. B, Structure of TgEndoG was analysed using the PyMOL Molecular Graphics System, Version 1.3r1-eduWin32 Schrödinger, LLC (collaboration with Antonio Jiménez-Ruiz, Universidad de Alcalá, Madrid). The active site for nucleic acid binding is indicated by a red circle. 


\subsubsection{T. gondii Endonuclease $G$ had a non-mitochondrial localisation}

MitoProt analysis, a software tool to predict a mitochondrial targeting of a protein, showed that TgEndoG is likely transported into mitochondria. However, YFP-tagged endogenous T. gondii EndoG (collaboration with Martin Thiele and Wolfgang Bohne, Göttingen) revealed that this protein has a non-mitochondrial localisation. YFP-tagged TgEndoG was detected using anti-YFP primary antibodies and fluorochrome-conjugated secondary antibodies (Figure 3.16A). To stain mitochondria, MitoTracker ${ }^{\circledR}$ Red CM$\mathrm{H}_{2} \mathrm{XRos}$ (Invitrogen) was used prior to labelling with anti-YFP antibodies (Figure 3.16B). These analyses also revealed that TgEndoG is constitutively expressed since it was already observed in single parasites and appeared not to be influenced by the size of the parasitophorous vacuole (Figure 3.16A).

A
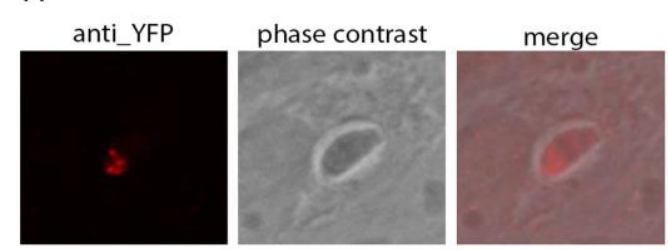

B
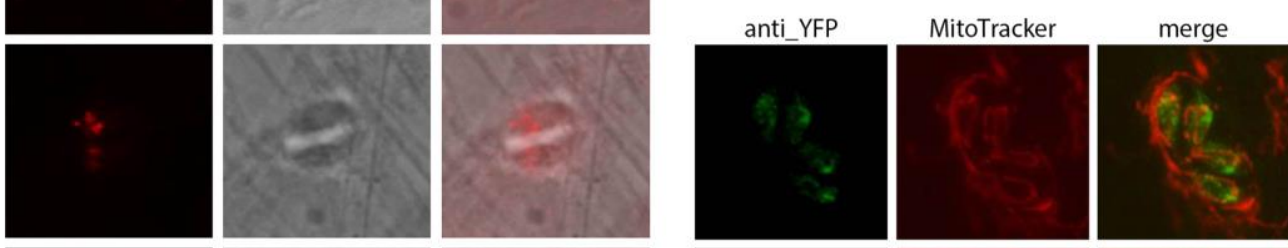

phase contrast
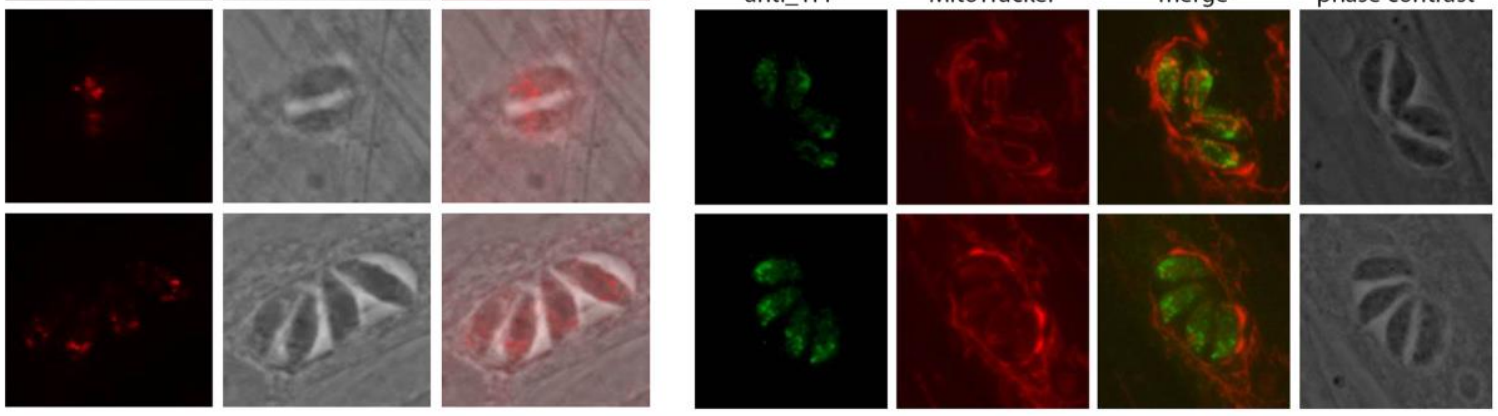

Figure 3.16. YFP-tagging of endogenous TgEndoG demonstrated that TgEndoG has a non-mitochondrial localisation. Tagging of TgEndoG with YFP and the detection were performed in collaboration with Martin Thiele and Wolfgang Bohne, Georg-August University of Göttingen. Images are kindly provided by Martin Thiele. A, Expression of TgEndoG (red fluorescence) was detected in single parasites and the intensity of TgEndoG expression did not change with the growth of the parasitophorous vacuole. B, Co-staining with MitoTracker (red fluorescence) showed that TgEndoG (green fluorescence) did not colocalise with mitochondria. 


\subsubsection{Cloning and expression of $T$. gondii Endonuclease $G$}

To further analyse the function of TgEndoG, molecular cloning of TgEndoG was performed. To this end, we generated a construct plasmid which contained the full length sequence of TgEndoG and heterologously expressed the recombinant protein in E. coli. After purification of the recombinant TgEndoG protein, it can be used for downstream experiments. In our study, the EndoG coding sequence was released from a construct plasmid TgEndoG-pBluescript SK- (synthetically generated; purchased from ATG:biosynthetics $\mathrm{GmBH}$ ) by digestion using EcoRI and KpnI and ligated into EcoRI- and KpnI-digested expression vector pASK-IBA3plus (Figure 3.17A). pASK-IBA3plus is an expression plasmid where expression of the recombinant protein is under transcriptional control of a tetracycline-regulated promoter. It also confers resistance against ampicillin. The protein will be a fusion with a Strep-tag at the C-terminus of the recombinant protein. Propagation of the plasmid was done in E. coli DH5a. Screening of positive clones were done by colony PCR and digestion using restriction enzymes. The correct sequences and orientation were further confirmed by DNA sequencing (see Chapter 2, section 2.2.4.4.1.7). For expression purposes the recombinant plasmids were transformed into $E$. coli BL21 codon plus. This strain of $E$. coli is designed for overcoming codon usage bias because it contains extra copies of genes encoding rare tRNAs, which often restrict the translation of heterologous proteins in E. coli (Rosano and Ceccarelli, 2009). We optimised the expression methods by modifying the concentration of anhydrotetracycline, ranging from 25, 50, 100, and $200 \mathrm{ng} / \mathrm{ml}$ anhydrotetracycline and also tested several growth temperatures $\left(30^{\circ} \mathrm{C}, 37^{\circ} \mathrm{C}\right.$ and room temperature) during induction of protein expression. However, despite these attempts to optimise protein expression we observed only a weak to moderate expression of TgEndoG using immunoblotting. The best condition for induction of expression of pASK-IBA3plus-TgEndoG in E. coli BL21 codon plus was observed using $25 \mathrm{ng} / \mathrm{ml}$ anhydrotetracycline at room temperature for $2 \mathrm{~h}$ (Figure 3.17B). 


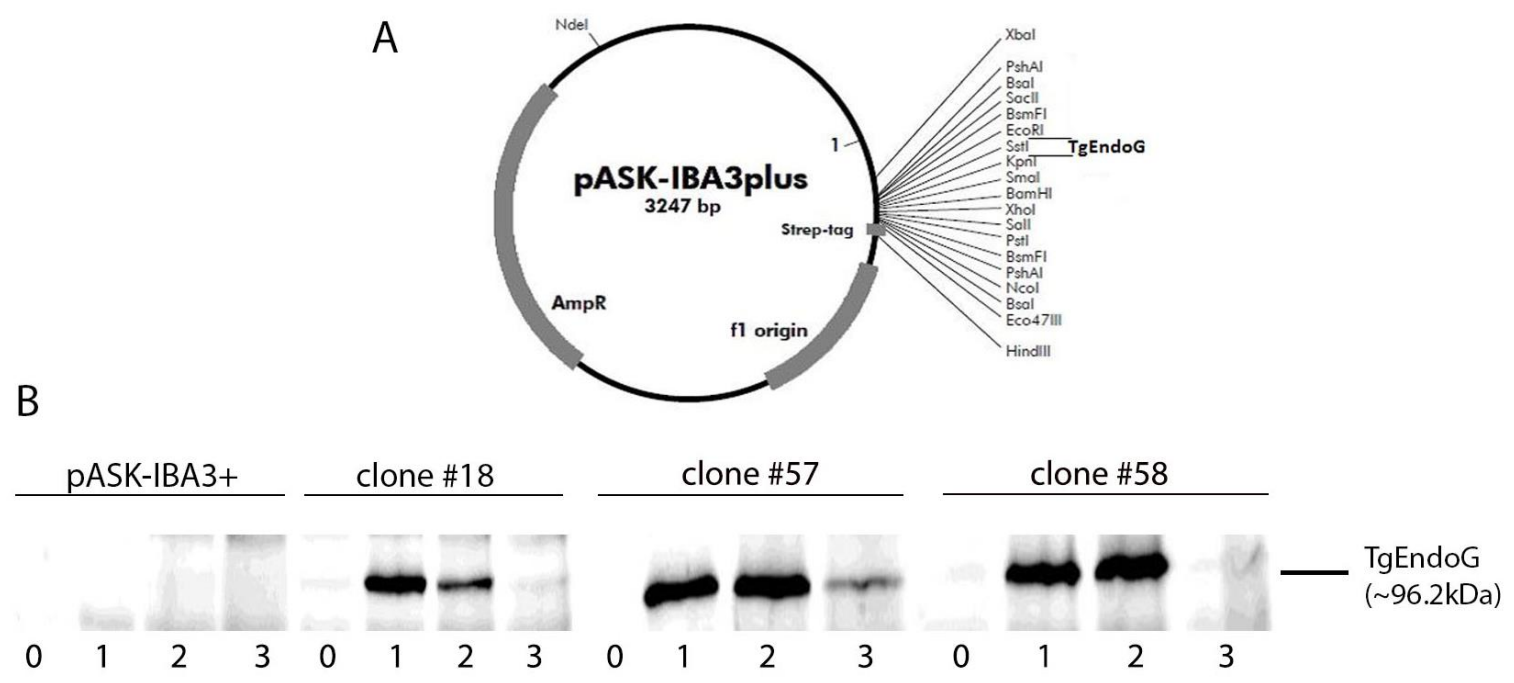

Figure 3.17. Recombinant plasmid construct of pASK-IBA3plus containing TgEndoG coding sequence and heterologous expression of the fusion protein as detected by immunoblotting. A, The construct of the recombinant plasmid pASK-IBA3plus containing the full length coding sequence of TgEndoG is indicated. B, The recombinant protein was expressed in E. coli BL21 codon plus using $25 \mathrm{ng} / \mathrm{ml}$ anhydrotetracycline at room temperature. TgEndoG was detected using anti-Strep-tag antibodies via immunoblotting and was visualised using a chemiluminescence imaging device (Luminescent Image Analyzer LAS-4000, Fujifilm). Representative immunoblots of negative control (contains only empty vector) and three positive clones are shown. $0,0 \mathrm{~h}$ (non-induced); 1, 2, 3, hour (s) post induction.

We also subcloned and expressed TgEndoG using other expression vectors, pRSET-A and pQE30. pRSET-A is an expression plasmid bearing an ampicillin resistant gene and which generates proteins fused with a 6xHis-tag at the N-terminus of the recombinant proteins. Expression of the recombinant protein is controlled by the strong bacteriophage T7 promoter. For expression of the gene of interest, sufficient T7 RNA polymerase that binds to the $\mathrm{T} 7$ promoter and transcribes the gene is needed; induction of the polymerase can be done using IPTG (Makrides, 1996). pQE30 also provides a 6xHistag and carries an ampicillin resistant gene. Instead of the T7 promoter, pQE30 has a T5 promoter, a native promoter. As T7, T5 is also an IPTG-inducible promoter (Makrides, 1996). Propagation of the plasmids containing TgEndoG was also done in E. coli DH5 $\alpha$. Transformants were screened by colony PCR and digestion using appropriate restriction enzymes (see Chapter 2, section 2.2.4.4.1.7). E. coli BL21 codon plus was again used for 
expressing of the protein. We optimised concentrations of IPTG, ranging from $0.5 \mathrm{mM}$ to $10 \mathrm{mM}$ and tested growth temperatures of $30^{\circ} \mathrm{C}, 37^{\circ} \mathrm{C}$, or room temperature. However, we could again not express TgEndoG protein in an amount that would allow downstream purification approaches. The best protein expression in this case was after induction with 2 $\mathrm{mM}$ IPTG at room temperature for $2 \mathrm{~h}$ (data not shown).

\subsection{Regulation of apoptosis-like cell death in $T$. gondii}

\subsubsection{Disruption of mitochondrial membrane potential following treatment of extracellular parasites with pro-apoptotic stimuli}

The absence of bona fide caspases and Bcl-2 family members in the Toxoplasma genome (Nedelcu, 2009 and our own bioinformatic search) suggested a caspaseindependent apoptosis-like cell death mechanism in T. gondii. Mitochondria play a central role in such pathways by releasing pro-apoptotic factors e.g., cytochrome c, AIF and EndoG following cell death stimuli. Thus, mitochondrial membrane potential $(\Delta \Psi \mathrm{m})$ is often considered an indicator of cellular health. MitoTracker staining and confocal microscopy was applied to determine $\Delta \Psi \mathrm{m}$ in $T$. gondii upon cell death induction.

To this end, extracellular parasites were incubated with staurosporine or miltefosine or were mock-treated for 24 and $48 \mathrm{~h}$. Freshly isolated as well as mock-treated parasites were used as controls. At the indicated time points, parasites were stained with MitoTracker® Orange CM- ${ }_{2}$ TMRos and analysed using confocal laser scanning microscopy. The number of parasites with an intact $\Delta \Psi \mathrm{m}$ was determined from at least 500 cells. Our result revealed that freshly isolated parasites accumulated the MitoTracker probe indicating an intact $\Delta \Psi \mathrm{m}$. Miltefosine-treated parasites showed a prominent disruption of the $\Delta \Psi \mathrm{m}$. The majority of parasites (more than $80 \%$ ) significantly lost their $\Delta \Psi \mathrm{m}$ already at $24 \mathrm{~h}$ post treatment (Figure 3.18; $\mathrm{p}<0.01$ ) and this decreased further until $48 \mathrm{~h}$ of treatment. In contrast, staurosporine treatment had no significant effect on the mitochondrial membrane potential as compared to mock-treated parasites (Figure 3.18). Thus, different cell death pathways appear to exist in $T$. gondii, one of which involves loss of $\Delta \Psi \mathrm{m}$ after miltefosine treatment. 
A

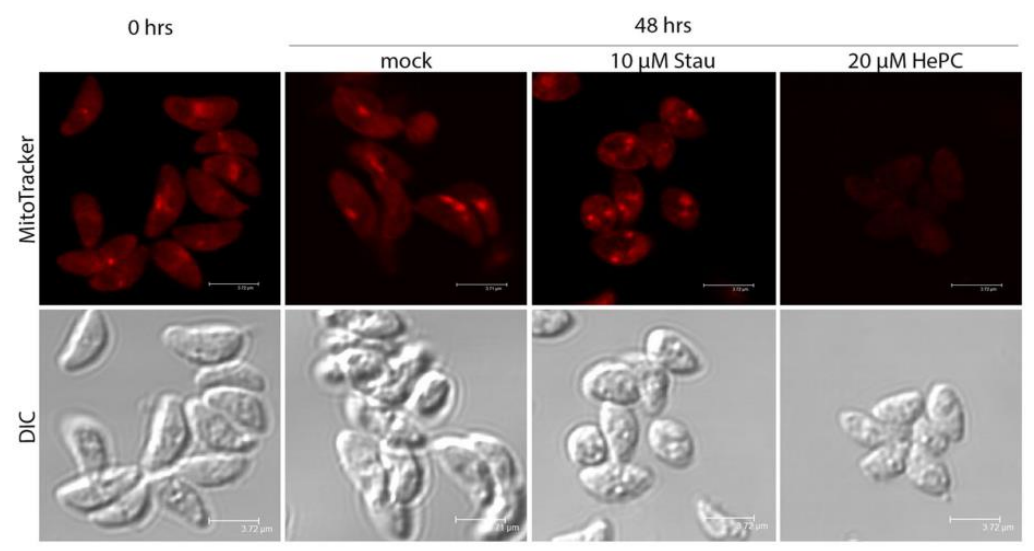

B

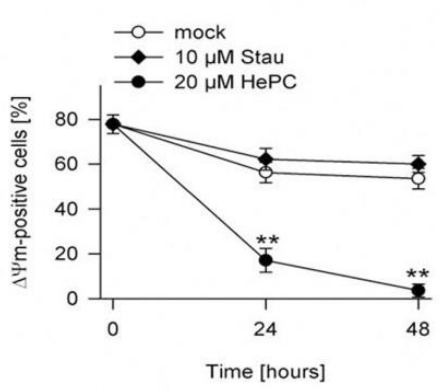

Figure 3.18. Dissipation of mitochondrial membrane potential $(\Delta \Psi \mathrm{m})$ after treatment of extracellular parasites with pro-apoptotic stimuli. Extracellular parasites were treated with $10 \mu \mathrm{M}$ staurosporine (stau) or $20 \mu \mathrm{M}$ miltefosine (HePC) or were mock-treated for 24 and $48 \mathrm{~h}$. At the indicated time points, parasites were stained with MitoTracker ${ }^{\circledR}$ Orange $\mathrm{CM}-\mathrm{H}_{2}$ TMRos A, Differential interference contrast (DIC) and fluorescence images were recorded by confocal laser scanning microscopy. Images are representative of extracellular parasites stained with MitoTracker (red labelling) at $48 \mathrm{~h}$ post treatment with pro-apoptotic stimuli. B, The graph shows the percentage of parasites with intact $\Delta \Psi \mathrm{m}$ at 24 and $48 \mathrm{~h}$ after pro-apoptotic treatment as determined from at least 500 cells per sample. Data represents means \pm S.E.M. from three independent experiments. ** $p<0.01$.

\subsubsection{Activation of caspase-like proteases was detected in $T$. gondii after treatment with pro-apoptotic stimuli}

Although protozoa do not express genuine caspases, i.e. crucial proteins for execution of apoptosis in mammals, caspase-like activities have been identified during PCD in several unicellular eukaryotes. Moreover, some proteases have been identified in the $T$. gondii genome and their expression was up-regulated upon cell death induction (see section 3.3.1). To test the possibility that caspase-like protease might be activated during cell death in T. gondii, CaspaTag assay and subsequent flow cytometry to identify activation of caspase-like proteases within cells was performed. To this end, extracellular parasites were cultivated in the presence of staurosporine and miltefosine and examined at various time points after treatment.

In situ labelling of parasites with a caspase inhibitor and flow cytometry revealed a strong and significant increase of caspase labelling within $2 \mathrm{~h}$ of miltefosine treatment 
(Figure 3.19; $\mathrm{p}<0.01)$. The caspase activity remained high at $4 \mathrm{~h}(\mathrm{p}<0.05)$ after miltefosine treatment before it declined to control levels until $48 \mathrm{~h}$ post treatment. Following staurosporine treatment, a significant increase in caspase labelling within $2 \mathrm{~h}$ of treatment as compared to mock-treated controls $(\mathrm{p}<0.01)$ was also observed. However, activation of caspase-like proteins was much lower than after miltefosine treatment and reached control levels already at 4 hours of treatment. Mock-treated control parasites did not show any significant increase in the caspase-like activity (Figure 3.19).

A

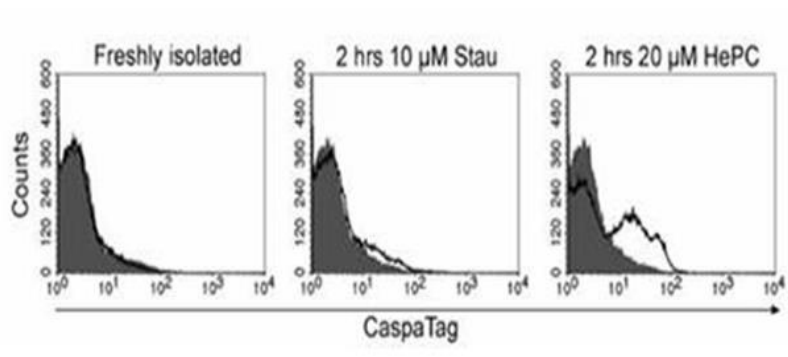

B

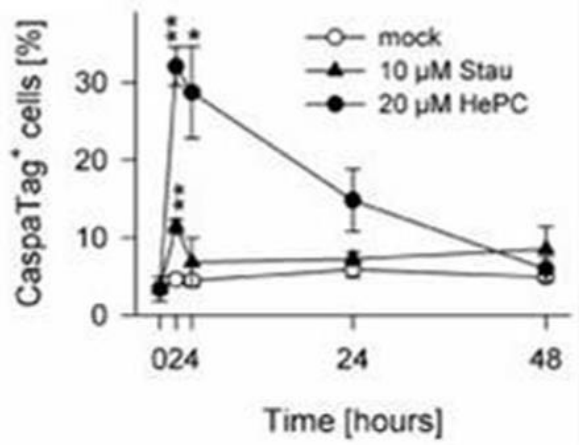

Figure 3.19. Caspase-like proteases were activated in T. gondii following pro-apoptotic treatment. Parasites were freshly isolated or were treated with $10 \mu \mathrm{M}$ staurosporine (stau), $20 \mu \mathrm{M}$ miltefosine (HePC), or were mock-treated for 2, 4, 24 and 48 h. Parasites were stained with a fluorescein-conjugated broad spectrum caspase inhibitor (CaspaTag) and analysed by flow cytometry. A, FACS histograms represent freshly isolated parasites or those treated with pro-apoptotic stimuli for $2 \mathrm{~h}$ (open histograms) as compared to mock-treated parasites (filled histograms). B, The graph shows the percentage of CaspaTag-positive parasites at different time points $(2$ to $48 \mathrm{~h}$ ) post treatment as revealed by flow cytometry. Data represents means \pm S.E.M. from three independent experiments. ** $p<0.01 ; * p<0.05$.

\subsubsection{Protease inhibitors prevented miltefosine-triggered cell death in extracellular parasites}

To further confirm an involvement of proteases in the regulation of apoptosis-like cell death in T. gondii, parasites were pre-treated with the cysteine protease inhibitor E64 or the pan-caspase inhibitor Z-VAD-FMK from $1 \mathrm{~h}$ prior to the induction of cell death with staurosporine or miltefosine onwards. The appearance of DNA fragmentation was then visualised using TUNEL assay and confocal laser scanning microscopy.

Emergence of DNA strand breaks as detected by TUNEL assay could be observed in $8.10 \%$ of untreated extracellular parasites. As expected from our previous results, 
treatments with staurosporine and miltefosine in the absence of both protease inhibitors significantly increased the number of TUNEL-positive parasites to $25.18 \%$ and $36.16 \%$, respectively (Figure 3.20; p<0.05). Protease inhibitors E64 and Z-VAD-FMK revealed a strong inhibition of the appearance of DNA strand breaks in parasites treated with miltefosine; the number of TUNEL-positive parasites significantly decreased to approximately $12 \%(\mathrm{p}<0.05)$. In contrast, both protease inhibitors did not significantly inhibit staurosporine-induced DNA strand breaks although we observed a trend of E64 to partially diminish DNA fragmentation following staurosporine treatment (Figure 3.20).

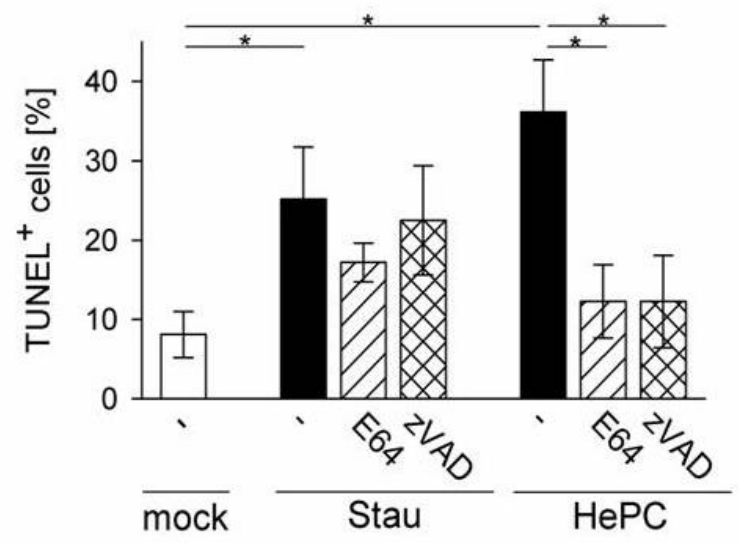

Figure 3.20. DNA strand breaks in T. gondii after treatment with miltefosine was inhibited by the presence of protease inhibitors. Parasites were treated with $100 \mu \mathrm{M}$ of protease inhibitors E64 or Z-VAD-FMK for $1 \mathrm{~h}$ prior to the induction of cell death with $10 \mu \mathrm{M}$ staurosporine (stau) or $20 \mu \mathrm{M}$ miltefosine (HePC). After $48 \mathrm{~h}$, DNA strand breaks were visualised by TUNEL staining. Number of TUNEL-positive parasites was counted from 500-800 cells per sample. Bars represent means \pm S.E.M. from three independent experiments. ${ }^{*} p$ $<0.05$.

Together, these results suggested the existence of different cell death pathways in $T$. gondii one of which involves loss of $\Delta \Psi \mathrm{m}$ and caspase-like proteases and which is specifically activated by miltefosine. 


\section{CHAPTER 4 \\ DISCUSSION}

\subsection{Cell death markers identified in $T$. gondii are characteristic for apoptosis in metazoans}

The concept that unicellular organisms undergo a form of programmed cell death which resembles apoptosis as described in metazoa is still a matter of debate. However, there are increasing numbers of reports describing the existence of an apoptosis-like cell death in single-celled organisms. The existence of apoptosis-like cell death in protozoan parasites indicated that parasites in a distinct host might organise themselves in populations and that communication might occur intercellularly between individuals (Duszenko et al., 2006; James and Green, 2004). Single-celled organisms could benefit from a self-killing mechanism in order to ensure the survival of a part of the population (Ameisen, 2002).

Apoptosis in multicellular organisms is characterised by specific morphological changes of a cell which include blebbing, condensation of the cell nucleus, degradation of nuclear DNA, and cell shrinkage (Kroemer et al., 2009). Apoptotic cells also exhibit several biochemical features such as internucleosomal cleavage of DNA, exposure of PS on the outer leaflet of the plasma membrane, and proteolytic cleavage of intracellular substrates (Elmore, 2007). Apoptotic cells are quickly removed by phagocytes before they can release their intracellular contents into the extracellular environment. Consequently, an inflammatory response will not be induced (Zimmermann et al., 2001).

Morphological and biochemical changes, e.g. cell shrinkage, phosphatidylserine exposure, DNA degradation, dissipation of the mitochondrial membrane potential $(\Delta \Psi \mathrm{m})$, or caspase-like activity have been identified following treatment with different proapoptotic stimuli in protozoan parasites including Leishmania, Trypanosoma, Plasmodium, Trichomonas, Blastocystis, Entamoeba, or Giardia (reviewed in Jiménez-Ruiz et al., 2010).

Here, we demonstrated that extracellular T. gondii shares several markers of apoptosis following treatment with common stimuli which are known to induce apoptosis in multicellular organisms. DNA fragmentation, a hallmark of apoptosis, was induced in $T$. 
gondii following staurosporine, miltefosine, $\mathrm{H}_{2} \mathrm{O}_{2}$, and camptothecin treatment. During apoptosis, nuclear DNA is cleaved by $\mathrm{Mg}^{2+}$ - and $\mathrm{Ca}^{2+}$-dependent endonucleases (Elmore, 2007) into DNA fragments with a 5'-phosphate and 3'-hydroxyl group (Nagata et al., 2003). Terminal deoxynucleotidyl transferase-mediated X-dUTP nick end labelling (TUNEL) assay was developed and has been widely used to label the cleaved DNA. We observed that the highest numbers of TUNEL-positive parasites were induced using staurosporine (a potent protein kinase inhibitor) and miltefosine (an antileishmanial agent).

Upon treatment with both stimuli, decreases in cell volume and rounding-up of the parasites have also been identified. Cell shrinkage is a regulated and rapid process during apoptosis, which is associated with loss of intracellular ions, particularly potassium through the $\mathrm{Na}^{+}, \mathrm{K}^{+}$-ATPase pump or the $\mathrm{Ca}^{2+}$-dependent $\mathrm{K}^{+}$channel (McCarthy and Cotter, 1997). Furthermore, rounding-up of cells ensues when cytoskeleton proteins are cleaved by activated proteases (Taylor et al., 2008).

Phosphatidylserine (PS) loses its asymmetric distribution in the lipid bilayer of the plasma membrane and is translocated from the inner to the outer leaflet of the plasma membrane during initiation of apoptosis (Jiménez-Ruiz et al., 2010). Two potential mechanisms have been suggested to explain this process. First, exposed PS occurs by the activation of a scramblase, an enzyme that is capable to randomise PS distribution across the plasma membrane. Second, exposure of PS is mediated by trafficking of lysosomes to the cell surface, a process that is mediated by calcium (Lee et al., 2013). Externalisation of PS acts as an "eat me" signal for surrounding cells (Kroemer et al., 2009). Binding of Annexin V to exposed PS has become an important tool for the evaluation of apoptosis. Here, we observed that in extracellular T. gondii, the percentage of Annexin V-positive cells significantly increased after treatment with staurosporine and miltefosine. Remarkably, the integrity of the plasma membrane (as detected using 7-AAD staining) was maintained, indicating apoptosis instead of necrosis.

Our results are consistent with a previous study which described that tachyzoites of T. gondii exhibited markers of apoptosis, e.g. chromatin condensation, DNA degradation and hypoploid nuclei after treatment with the exogenous nitric oxide (NO) donor, sodium nitroprusside (Peng et al., 2003). It has been also shown that parasites exposed their PS to inhibit NO production (which contributes to the control of $T$. gondii growth) to ensure its 
persistence in activated macrophages (Seabra et al., 2004) or to facilitate parasite and host survival (Santos et al., 2011).

Importantly, intracellular T. gondii also showed DNA strand breaks following treatment with staurosporine, but not miltefosine, at 3 and $6 \mathrm{~h}$ post treatment. The absence of DNA fragmentation during early time points of miltefosine treatment does not rule out an effect of this drug on intracellular parasites. It was described that the parasiticidal effect of miltefosine on intracellular Neospora caninum was only observed after $20 \mathrm{~h}$ of treatment (Debache and Hemphill, 2012); thus, the effect of miltefosine on intracellular $T$. gondii for longer time periods (or with higher concentration of miltefosine) awaits clarification.

Staurosporine and miltefosine have also been used to induce apoptosis in multicellular organisms. For instance, staurosporine induced apoptosis in L1210/0 and L1210/S cells (a carcinogen-induced acute lymphoid leukemia from DBA mice) via caspase-dependent and caspase-independent pathways (Belmokhtar et al., 2001). Miltefosine induced apoptosis in a variety of tumour cell lines and Leishmania spp., possibly via perturbation of fatty acid and sterol metabolism, mitochondrial dysfunction and/or inhibition of the Akt/PKB pathway (Dorlo et al., 2012)

Collectively, our data strongly indicated that following treatment with pro-apoptotic stimuli, T. gondii can undergo a form of programmed cell death that shows characteristic features of apoptosis as observed in metazoa.

\subsection{Possible benefits of an apoptosis-like cell death in $T$. gondii}

Apoptosis in metazoa is an essential mechanism to remove cells without inducing an inflammatory response. It fulfills crucial functions in a wide range of physiological processes, for instance during embryonic development, to maintain tissue homeostasis, to eliminate damaged or unwanted cells, or as a defence mechanism against infection (Ameisen, 2002; Proto et al., 2013). If an apoptosis-like cell death in protists does exist, a major question that arises is what the advantages for protozoan parasites to commit suicide are. This question is critical since a cell death programme would only evolve if it is beneficial for the survival of the parasites. 
An apoptosis-like cell death arguably plays essential roles in regulating parasites densities in distinct host compartments and in avoiding inflammatory responses to ensure a perpetuation of infection (van Zandbergen et al., 2010). For instance, Trypanosoma cruzi undergoes apoptosis in order to control cell proliferation and ensure the survival of the parasite population as a whole (Ameisen et al., 1995). Another example has been described in a malaria parasite, Plasmodium berghei; the parasite dies naturally by apoptosis in the mosquito midgut before invading the intestinal epithelium to limit intensity of infection (Al-Olayan et al., 2002).

In our study, we elucidated at what time T. gondii, during its natural life cycle, undergoes an apoptosis-like cell death. We found that DNA fragmentation, as detected by TUNEL staining, was not prevalent during the acute stage of infection in the intraperitoneal cavity of $T$. gondii-infected mice. However, we identified TUNEL-positive bradyzoites of $T$. gondii within tissue cysts from isolated brains of chronically infected mice. Thus, the results suggested that an apoptosis-like cell death in $T$. gondii did not contribute during acute infection, but may play a role during chronic infection. Our results were not in line with a previous study that described a $T$. gondii parasite population exposing PS on the outer leaflet of the plasma membrane in the peritoneal fluid of mice during acute infection (Seabra et al., 2004). However, since DNA fragmentation occurs in the later phase of apoptosis (Elmore, 2007), the absence of TUNEL-positive parasites in the peritoneal cavity of infected mice, as we detected it here, did not completely rule out the potential that cells were in the early stage of apoptosis.

Purposes of an apoptosis-like cell death in $T$. gondii, whether altruistic or otherwise, are still an issue to be elucidated. Altruistic suicide with respect to nutrient supply (James and Green, 2004) is unlikely for bradyzoites of T. gondii because this stage grows slowly and the metabolism is strongly reduced as compared to the tachyzoite stage. Moreover, we need to note that $T$. gondii is an obligate intracellular pathogen. One problem that is commonly faced by an intracellular organism is how to ensure sufficient space for replication (Patricia Leiriao, 2004). Therefore, one possible advantage of an apoptosis-like cell death in bradyzoites apparently is limiting the population size within tissue cysts and consequently avoiding damage of the host cell and thus ensuring the survival of the viable bradyzoites. Death of the host cells might lead to death of 
bradyzoites since intact host cells provides a continuous supply of nutrient (Pavesio et al., 1992). On the other hand, rupture of tissue cysts is occasionally observed and is accompanied with an inflammatory response of the host (Dubey et al., 1998). A subpopulation of bradyzoites with apoptotic features might also limit the immune response of the host and thereby facilitating the viable bradyzoites to develop into new cysts. As described in Leishmania major, the presence of apoptotic parasites can silence polymorphonuclear (PMN) cell functions by releasing the anti-inflammatory cytokines TGF- $\beta$ and IL-10 thereby enabling viable parasites to develop a productive infection (van Zandbergen et al., 2006).

Importantly, toxoplasms in a distinct host are a clonal population since they regularly originate from a single inoculum transferred by ingestion of undercooked meat from a chronically infected live stock (containing bradyzoites within tissue cysts) (Tenter et al., 2000). Furthermore, acute T. gondii infection provokes a strong Th1-type immunity that restricts dissemination of parasites and allows the survival of the host but which also mostly prevents a productive super-infection with a second $T$. gondii strain. Consequently, bradyzoites are formed, predominantly in the muscle and brain tissues (Filisetti and Candolfi, 2004). Thus, if we consider T. gondii as a population of genetically identical organisms within its host, a form of programmed cell death of a subpopulation of parasites may be evolutionary sustainable because it could help avoiding an overwhelming parasite load and host death before parasite transmission would have been possible (Luder et al., 2010). This may be particularly important for the $T$. gondii transmission cycle which critically depends on establishing a long-lasting chronic infection.

Taken together, our findings exhibited that an apoptosis-like cell death might play roles during chronic infection of $T$. gondii. Possibly, it is responsible for the regulation of bradyzoite density within tissue cysts or to avoid overwhelming immune responses of the host when bradyzoites are released as a result of tissue cyst rupture.

\subsection{Identification of putative regulators of an apoptosis-like cell death in $T$. gondii}

Caspases and Bcl-2 family proteins play essential roles in the regulation and execution of apoptosis in multicellular organisms. Since $T$. gondii does not express bona 
fide caspases or members of Bcl-2 proteins, an apoptosis like cell death in $T$. gondii is assumed to occur in a caspase-independent fashion.

We identified 17 genes via an in silico analysis of the Toxoplasma genome database with annotated functions in programmed cell death and which were differentially expressed following pro-apoptotic treatment of the parasite. Virtually all mRNAs of the seventeen selected genes increased in a time-dependent fashion following staurosporine treatment of $T$. gondii. We observed an up-regulation of the mRNAs by more than 10-fold for PDCD2 (TgME49_105490), a putative ELMO (TgME49_053000), a DNA/RNA endonuclease (TgME49_008710), a putative Fas apoptotic inhibitory molecule (TgME49_049770) and two calpain-like proteins. A strong up-regulation of the transcript of PDCD2 was also observed after treatment with miltefosine while other transcripts were only slightly up-regulated, or even not at all in response to miltefosine.

Increased PDCD2 expression can induce apoptosis in human cells, at least in part by activating a caspase cascade, whereas repression of PDCD2 is involved in the pathogenesis of certain human lymphomas (Baron et al., 2002; Baron et al., 2010). It is evolutionarily conserved and has been identified in all eukaryotes (Nedelcu, 2009). The ELMO protein plays roles in the engulfment and elimination of apoptotic cells in mammals, a final step of the apoptosis process (Gumienny et al., 2001). Calpains, as caspases, are cysteine proteases but compared to caspases, calpains show a broad spectrum with respect to cleavage specificity. Calpains, in mammalian cells, cleave a large number of proteins during apoptosis; calpains act together with caspases or might also cleave proteins independently of caspases or in combination with other proteins such as AIF (apoptosis inducing factor) (Vandenabeele et al., 2005). The DNA/RNA endonuclease (in our study named TgEndoG) and putative Fas apoptotic inhibitory molecules (in our study named TgBIs) are of our interest considering that both of these proteins are known to play critical roles in caspase-independent apoptosis pathways (described in more detail below).

Bax inhibitor-1 (BI-1) was initially described as testis enhanced gene transcript (TEGT), and has been cloned from adult rat testis. The predicted sequence of human BI-1 revealed a $90 \%$ identity to TEGT (Watanabe and Lam, 2009). It contains 6 to 7 transmembrane domains and is capable to inhibit cell death in animal and plant cells (Huckelhoven, 2004). The protein is mainly localised to ER membranes, but can also be 
detected at mitochondria (Chae et al., 2004; Xu and Reed, 1998). BI-1 does not directly interact with Bax (a pro-apoptotic protein of the Bcl-2 family), but exerts its anti-apoptotic function by an interaction with anti-apoptotic members of the Bcl-2 protein family, i.e. Bcl-2 and Bcl- $\mathrm{X}_{\mathrm{L}}$ (Watanabe and Lam, 2009; Xu and Reed, 1998). This view is supported by the fact that plants have BI-1 even though Bax cannot be identified in plant genomes (Huckelhoven, 2004). There are no well-characterised motifs for post-translational modification of BI-1, however the C-terminus of the protein contains a lysine-rich motif (EKDKKKEKK) that resembles the pH-sensing motif of ion channels (Kim et al., 2008; Watanabe and Lam, 2009).

The T. gondii genome revealed the presence of three putative Bax-inhibitors and they were named TgBI-1, TgBI-2 and TgBI-3, respectively. Analysis of TgBIs sequences exhibited no particular motif that could be used to predict their function. After heterologous expression in HeLa cells, immunofluorescence assays using anti-FLAG antibody recognising the FLAG tag fusion proteins of TgBIs showed that TgBI-1 has a perinuclear localisation, TgBI-2 has a predominantly cell surface expression and TgBI-3 has both a perinuclear and a cell surface localisation. It is not surprising that these three proteins have different localisations, since Bax-inhibitors in higher eukaryotes reside in different locations (Chae et al., 2004; Xu and Reed, 1998). However, our results showed evolutionary conserved protective effects of the BI proteins from T. gondii to mammals. Heterologous expression of TgBIs in a well-described mammalian apoptosis system, namely HeLa cells, showed an inhibition of caspase activity by TgBI-1 and TgBI-2, but not TgBI-3, following treatment with staurosporine (a potent protein kinase inhibitor) and brefeldin A (an inhibitor of protein transport from the ER to the Golgi apparatus), suggesting that TgBIs impact downstream cell death pathways which involve caspases. Moreover, TgBIs in HeLa cells partially prevented the appearance of hypoploid DNA after treatment of TgBIs-transfected HeLa cells with staurosporine and brefeldin A. A decrease of the DNA content caused by apoptosis, so-called hypoploid DNA (Krysko et al., 2008) is a common feature of apoptosis that can be also identified as a consequence of caspaseindependent pathways of apoptosis (Jones et al., 1999). Although the role of TgBIs in the regulation of apoptosis in $T$. gondii remains elusive, it is possibly crucial in the regulation of ER-associated apoptotic cell death. It has been described that accumulation of unfolded 
proteins in the lumen of the ER (ER stress) induced by oxidative stress, $\mathrm{Ca}^{2+}$ homeostasis disruption or hypoxia triggers an apoptotic cell death in mammals (Xu et al., 2005). Stress in the ER induces a mitochondrial-dependent apoptosis pathway or activation of caspase 12 which consequently activates the caspase cascade, leading to cell death (Breckenridge et al., 2003; Xu et al., 2005). In mammalian cells, BI-1 suppressed the ER stress response under mild ER stress conditions, indicating that BI-1 function is necessary during the early stage of ER stress adaptation to prevent activation of apoptosis (Lisbona et al., 2009). Moreover, BI-1 also plays a role in regulating $\mathrm{Ca}^{2+}$ homeostasis in the ER (Robinson et al., 2011).

Another protein that is involved in a caspase-independent apoptosis pathway in mammals is EndoG. Endonuclease G was initially defined as an endonuclease that specifically cleaves guanine $(\mathrm{G})$ tracts in DNA extracts of chicken erythrocytes even though later it was recognised that the protein is also able to induce DNA nicks within cytosine tracts (Low, 2003). The protein belongs to a large family of DNA/RNA nonspecific nucleases. Nucleases of this family are expressed in many prokaryotes and eukaryotes including bacteria, protozoa, fungi, slime moulds, invertebrates and vertebrates (Schafer et al., 2004). The active site is characterised by a DGRH motif that contains a critical histidine residue and is structurally part of the $\beta ß \alpha$-Me-finger motif. EndoG requires $\mathrm{Mg}^{2+}$ or other divalent cations for its activity (Rico et al., 2009; Schafer et al., 2004). Genes encoding EndoG have also been identified in Leishmania infantum (Rico et al., 2009) and Trypanosoma (Gannavaram et al., 2008) and share 30\% identity with human EndoG. The predicted nuclease domains of Leishmania and Trypanosoma EndoG displayed $48 \%$ identity to human EndoG, in which aspartic acid at the DRGH motif of mammalian EndoG is replaced by serine (DRGH is substituted by a SRGH motif). In mammalian cells, EndoG is translocated from mitochondria to the nucleus following treatment with apoptotic stimuli, leading to oligonucleosomal DNA fragmentation independently of caspases (Arnoult et al., 2003; Schafer et al., 2004). Putative EndoG of Leishmania and Trypanosoma also showed pro-apoptotic nuclease activity; the protein, following cell death induction, was released from mitochondria and translocated into the nucleus consequently leading to cell death (Gannavaram et al., 2008; Rico et al., 2009). 
The predicted T. gondii EndoG (TgEndoG) amino acid sequence shared $31.5 \%$ identity with human EndoG. Prediction of the TgEndoG structure using PyMOL showed that TgEndoG structurally resembles those of Endonuclease $G$ from other species. The DRGH motif in the active site of human EndoG or the SRGH motif in the active site of EndoG from Leishmania and Trypanosome is replaced by a SKGH motif in TgEndoG. Importantly, however, the histidine and asparagine residues which are important for catalytic activities and divalent ion binding respectively, are conserved between TgEndoG and that from Leishmania and Trypanosoma (Gannavaram et al., 2008). Interestingly, EndoG in other species is known to reside in mitochondria, but TgEndoG has a nonmitochondrial localisation as detected by tagging of endogenous TgEndoG and MitoTracker co-staining; the protein showed a vesicular morphology and was dispersed within the parasite cytosol. We have successfully cloned the gene encoding TgEndoG but we still need to optimise the expression method to obtain an adequate amount for purification and downstream experiments. Precise localisation and purification of TgEndoG is also critical to be addressed in order to further elucidate whether the TgEndoG might have functions in an apoptosis-like cell death mechanism in T. gondii. This view is supported by the facts (i) that EndoG showed pro-apoptotic nuclease activity in other protozoan parasites, (ii) that the predicted structure of TgEndoG showed a conservation to the active domain of EndoG (histidine and asparagine residues) in metazoans, and (iii) that we detected DNA fragmentation in $T$. gondii following treatment with pro-apoptotic stimuli in the absence of caspases, suggesting that EndoG might be involved.

Together, we have identified 17 cell death-associated genes via in silico analysis from the Toxoplasma genomic database, which were differentially expressed following pro-apoptotic treatment of the parasite. TgBIs and TgEndoG are of interest. Our findings on TgBIs indicated evolutionary conserved protective effects of BI proteins from $T$. gondii to mammals and suggested a role of $\mathrm{TgBI}$ proteins in the regulation of apoptosis-like cell death in T.gondii.

\subsection{The existence of distinct pathways of an apoptosis-like cell death in $T$. gondii}

Although similarities clearly exist between protozoa and higher eukaryotes in some features of apoptosis and apoptosis-related molecules, molecular pathways of apoptosis- 
like cell death in protozoan parasites have not yet been convincingly identified. However, it is quite obvious that the mechanism of apoptosis in parasites and higher eukaryotes differs (Debrabant and Nakhasi, 2003).

In our study, we found clear evidence that distinct apoptosis-like cell death pathways were induced in $T$. gondii in response to staurosporine and miltefosine treatments, respectively. Following treatment of extracellular parasites with miltefosine, a prominent loss of the mitochondrial membrane potential $(\Delta \Psi \mathrm{m})$ was detected. Conversely, staurosporine treatment had no significant effect on $\Delta \Psi \mathrm{m}$.

Mitochondria in vertebrates are crucial in regulating cell death through different mechanisms, e.g. via transducing upstream apoptotic signals into the caspase cascade or by releasing caspase-independent pro-apoptotic molecules such as EndoG and AIF (Wang and Youle, 2009). Collapse of the mitochondrial membrane potential was considered a point of no return in the apoptosis pathway (Jeong and Seol, 2008). Disruption of the mitochondrial membrane potential has been associated with apoptosis-like cell death in other organisms, such as Leishmania and Trypanosoma (Arnoult et al., 2002; Figarella et al., 2005) and Dictyostelium discoideum (Arnoult et al., 2001).

T. gondii possesses a single mitochondrion; it is located in the anterior part of the protozoan body, is projected to the posterior region, and is considered to be crucial during the invasion process by the parasite (Melo et al., 2000). Here, we found that the mitochondrion of $T$. gondii is involved in a form of programmed cell death in the parasite especially after miltefosine treatment. However, importantly, it should be pointed out that the intact $\Delta \Psi \mathrm{m}$ as observed after staurosporine treatment does not exclude an involvement of mitochondria in staurosporine-triggered apoptosis-like cell death in $T$. gondii since loss of $\Delta \Psi \mathrm{m}$ is also not necessarily crucial for MOMP in mammals (Kroemer et al., 2007) and for execution of apoptosis in invertebrates (Wang and Youle, 2009; Zimmermann et al., 2002). In addition, involvement of $\Delta \Psi \mathrm{m}$ in Plasmodium apoptotic pathways is also a matter of debate (Jiménez-Ruiz et al., 2010) .

Cysteine proteases appeared crucial during cell death induction of $T$. gondii by miltefosine. Here, we found a strong increase of CaspaTag-positive parasites within two hours of miltefosine treatment. CaspaTag used in our study is a non-cytotoxic fluorescent caspase inhibitor (FAM-VAD-FMK) that binds covalently to active caspases in living 
cells. It should be noticed that VAD-FMK inhibits a broad spectrum of caspases; this implies that VAD-FMK does not only bind to genuine caspases. As described in Leishmania parasites, Z-VAD-FMK could bind to the cathepsin B-like protein (clan CA cysteine protease), explaining the detection of caspase binding in organisms which do not have genes encoding caspases (El-Fadili et al., 2010). Additionaly, VAD-FMK can also bind to legumain (clan CD cysteine protease) (Rozman-Pungercar et al., 2003). Thus, this may explain the efficient binding of VAD-FMK in $T$. gondii treated with miltefosine although a bona fide caspase in the T. gondii genome could not be identified (Nedelcu, 2009 and our own bioinformatics analysis). Five genes encoding papain family cathepsins from $T$. gondii, including three cathepsin Cs (TgCPC1, 2, 3), one cathepsin B (TgCPB) and one cathepsin L (TgCPL), have been characterised in T. gondii (Que et al., 2007). These proteins have been described to play important roles in maturation of excretory/secretory proteins, in the invasion process and in parasite replication (Parussini et al., 2010; Que et al., 2007; Que et al., 2002); whether they have a role in T. gondii PCD has not yet been reported. Moreover, the crucial role of cysteine proteases in the regulation of an apoptosislike cell death in $T$. gondii following miltefosine treatment is supported by our findings that a cysteine protease inhibitor (E64) or a pan-caspase inhibitor (Z-VAD-FMK) strongly decreased the appearance of TUNEL-positive parasites after treatment of extracellular parasites with miltefosine. Treatment of parasites with staurosporine also induced a strong increase of caspase-like activity within two hours of treatment. However, in contrast, both protease inhibitors did not significantly inhibit staurosporine -induced DNA fragmentation although we observed a trend of E64 to partially diminish DNA strand breaks following staurosporine treatment.

Our results indicated that different signalling pathways were activated in $T$. gondii in response to different pro-apoptotic stimuli as summarised in Table 4.1. 
Table 4.1. Regulation of an apoptosis-like cell death in $T$. gondii following staurosporine and miltefosine treatment

\begin{tabular}{|c|c|c|}
\hline & Staurosporine & Miltefosine \\
\hline Mitochondria & Intact $\Delta \Psi \mathrm{m}$ & Loss of $\Delta \Psi \mathrm{m}$ \\
\hline Caspase-like activity & $\begin{array}{l}\text { Small but significant } \\
\text { increase at } 2 \text { hours }\end{array}$ & $\begin{array}{l}\text { Strong increase at } 2 \text { and } 4 \\
\text { hours }\end{array}$ \\
\hline $\begin{array}{l}\text { Protease inhibitors (E64, } \\
\text { zVAD) }\end{array}$ & No significant impact & Prevention of cell death \\
\hline $\begin{array}{l}\text { Up-regulation of mRNAs } \\
\text { (>10-fold) }\end{array}$ & $\begin{array}{l}\text { PDCD2, ELMO, DNA/RNA } \\
\text { endonuclease, Fas apoptotic } \\
\text { inhibitor, calpain-like } \\
\text { proteins }\end{array}$ & PDCD2 \\
\hline $\begin{array}{l}\text { Downstream apoptotic } \\
\text { markers }\end{array}$ & $\begin{array}{l}\text { DNA strand breaks } \\
\text { (TUNEL), PS exposure, } \\
\text { no membrane } \\
\text { permeabilization, pyknosis, } \\
\text { rounding-up of cell }\end{array}$ & $\begin{array}{l}\text { DNA strand breaks } \\
\text { (TUNEL), PS exposure, } \\
\text { no membrane } \\
\text { permeabilization, pyknosis, } \\
\text { rounding-up of cell }\end{array}$ \\
\hline
\end{tabular}

\subsection{T. gondii underwent apoptosis-like cell death during drug treatment}

Here we identified for the first time that extracellular parasites underwent a form of cell death with the features of apoptosis, i.e. DNA fragmentation, during treatment with common drugs used to treat toxoplasmosis. DNA strand breaks, as detected by TUNEL assay, were most prominently and significantly induced in extracellular tachyzoites of $T$. gondii during atovaquone treatment. Atovaquone (hydroxynaphthoquinone) is an analogue of the inner mitochondrial coenzyme ubiquinone (coenzymeQ), which is an integral component of the mitochondrial electron transport chain. Atovaquone inhibits binding of ubiquinone to cytochrome $b c 1$ (complex III) and consequently leads to collapse of the mitochondrial membrane potential (Baggish and Hill, 2002; McFadden et al., 2000). However, atovaquone induced only very low levels of DNA strand breaks in intracellular parasites as shown herein. DNA strand breaks in intracellular parasites, intriguingly, were significantly induced by clindamycin (a lincosamide) during the second cycle of infection. This was accompanied with a significant reduction of the number of parasites per 
parasitophorous vacuole. It has been reported that clindamycin has a remarkable delayed lethal effect on T. gondii in vitro (Fichera et al., 1995; Pfefferkorn et al., 1992). Inhibition of $T$. gondii growth by clindamycin via an irreversible toxic effect on the host cell was excluded previously (Pfefferkorn et al., 1992), largely excluding the possibility that induction of DNA strand breaks in $T$. gondii occurred by an indirect mechanism mediated by the host cell. In $T$. gondii, clindamycin targets the apicoplast (a chloroplast-like organelle) protein translation by binding to the apicoplast large-subunit rRNA (Camps et al., 2002). However, it should be noted that only a minor percentage of parasitophorous vacuole contained TUNEL-positive intracellular parasites after treatment of intracellular parasites with clindamycin. This may be due to the fact that parasites are mainly sensitive to clindamycin during the very late stages of endodyogeny (Camps et al., 2002).

Together, these results demonstrated that an apoptosis-like cell death phenotype could be induced in extracellular and intracellular $T$. gondii following treatment with commonly used chemotherapeutic drugs against toxoplasmosis.

Finally, our findings support a concept that unicellular organisms can undergo a form of programmed cell death, e.g. apoptosis. Herein we apply a combination of several techniques in order to unravel the existence of an apoptosis-like cell death in T. gondii. We proof that characteristic features of apoptosis, including DNA strand breaks, decrease in cell volume and externalization of PS can be identified in T. gondii. We also provide evidence that an apoptosis-like cell death mechanism in $T$. gondii might play a role during chronic infection. Importantly, treatment of intracellular parasites with clindamycin also induced an apoptosis-like cell death phenotype in a minor proportion of parasites. Using in silico analysis of the Toxoplasma genome resource, we identified 17 genes with annotated functions in programmed cell death and which were differentially expressed following proapoptotic treatment of the parasite. Our focus on TgBIs indicated evolutionary conserved protective effects of BI proteins from $T$. gondii to mammals and suggested a role of $\mathrm{TgBI}$ proteins in the regulation of apoptosis. Furthermore, our data indicated that T. gondii developed distinct apoptosis-like cell death mechanisms in response to different proapoptotic stimuli. One involves loss of the $\Delta \Psi \mathrm{m}$ and activation of caspase-like proteases, which was specifically triggered by miltefosine. Further experiments to unravel the precise 
mechanisms of apoptosis-like cell death in Toxoplasma are required. If an apoptosis-like cell death does exist in protozoan parasites, exploiting the process would give promising insights into the evolution of death pathways and may reveal novel strategies to combat infectious diseases of high impact on human and animal health. 


\section{SUMMARY}

Toxoplasma gondii is an apicomplexan protozoan parasite which leads to widespread infections in humans and animals worldwide. The mechanisms of how T. gondii dies are largely unknown but knowledge of putative regulated cell death pathways are of major scientific interest and may lead to the development of novel antiparasitic therapies. Apoptosis, i.e. a form of programmed cell death (PCD), is a common feature of multicellular organisms but there is increasing evidence of apoptosis-like cell death pathways in unicellular organisms, including protozoan parasites. Aims of this study were to characterise PCD phenotypes in T. gondii, to unravel possible roles of PCD in T. gondii, to identify molecules that are responsible for PCD in $T$. gondii, and to characterise the regulation of cell death in $T$. gondii.

Terminal deoxynucleotidyl transferase-mediated X-dUTP nick end labelling (TUNEL) assays followed by flow cytometry were used to determine DNA strand breaks after treatment of the parasite with pro-apoptotic inducers. Basal levels of DNA strand breaks were detected in freshly isolated and untreated parasites. However, staurosporine and miltefosine significantly increased the number of TUNEL-positive extracellular parasites in a time-dependent manner from 24 to 72 hours post treatment. Staurosporineand miltefosine-treated parasites also showed a reduction in cell size and a rounding-up morphology as compared to untreated parasites which is also indicative for an apoptosislike cell death. Furthermore, treatment with staurosporine and miltefosine led to a higher percentage of Annexin V-positive parasites exposing phosphatidylserine on the cell surface as compared to untreated controls whereas the plasma membrane integrity was not compromised. Importantly, treatment of parasite-infected human fibroblasts with staurosporine induced the appearance of TUNEL-positive intracellular parasites, indicating that an apoptosis-like cell death can also occur in parasites in their natural intracellular environment.

In our study, we did not find evidence that apoptosis-like cell death contributes to the regulation of tachyzoite density during acute infection in the peritoneal cavity of infected mice. However, TUNEL-positive parasites were detected in bradyzoite-containing 
tissue cysts from the brains of chronically infected mice, suggesting that $T$. gondii employed an apoptosis-like cell death during chronic infection. We further investigated whether parasites underwent apoptosis under treatment with various drugs commonly used for chemotherapy of toxoplasmosis. Parasite replication decreased after treatment with anisomycin, pyrimethamine, atovaquone, and clindamycin, as shown by lower numbers of parasites per parasitophorous vacuole as compared to the untreated parasites. The number of extracellular TUNEL-positive parasites increased after treatment with all drugs. Intriguingly, treatment with clindamycin resulted in the emergence of intracellular TUNEL-positive parasites, suggesting that drug treatment can also induce an apoptosislike phenotype in intracellular T. gondii.

In order to identify proteins which might play a role in $T$. gondii $\mathrm{PCD}$, seventeen cell death-related genes were identified in the T. gondii genome database. Messenger RNA analysis of these genes showed that all of them were expressed in untreated parasites and that several of them were strongly up-regulated upon staurosporine treatment. Miltefosine treatment led to a more restricted transcriptional response of $T$. gondii with a putative PDCD2 gene again, as in response to staurosporine, being most strongly up-regulated. In this project, we focused on T. gondii Bax inhibitors and Endonuclease G, conserved proteins which might play roles in ancient PCD pathways.

In silico analysis of the $T$. gondii genome demonstrated the presence of putative Bax inhibitor genes, named T. gondii Bax inhibitor-1, -2 and -3 (TgBI-1, TgBI-2 and TgBI-3). Amino acid sequence analysis of TgBI-1, TgBI-2 and TgBI-3 showed 33-38 \% identity with human BI-1. Putative TgBI-1, TgBI-2 and TgBI-3 contain 6-9 transmembrane domains and have a molecular weight of 37,974 Da, 29,322 $\mathrm{Da}$ and 37,345 $\mathrm{Da}$, respectively. To unravel possible functions of TgBIs in the regulation of apoptosis, we stably transfected HeLa cells with the genes encoding TgBI-1, TgBI-2 and TgBI-3 and this was confirmed by immunofluorescence microscopy. Measurement of caspase 3/7 activity demonstrated an inhibitory effect of TgBI-1 and TgBI-2 in HeLa cells after treatment with staurosporine and brefeldin A. Moreover, TgBI-1, TgBI-2- and TgBI-3-expressing HeLa cells showed a lower induction of hypoploid DNA (subpeak G0/G1) after treatment with pro-apoptotic stimuli as above. These results suggested that $T$. gondii Bax inhibitors might fulfill conserved functions in regulating an apoptosis-like cell death in $T$. gondii. 
T. gondii Endonuclease G (TgEndoG) shares $31.5 \%, 38.1 \%, 36.1 \%$ and $35.3 \%$ identity with EndoG of Homo sapiens, Mus musculus, Bos taurus and S. cerevisiae respectively. Putative TgEndoG consists of 894 amino acids and has a molecular weight of $96.2 \mathrm{kDa}$. Using MitoProt analysis, this protein is likely transported into mitochondria. However, YFP-tagged endogenous TgEndoG revealed that this protein has a nonmitochondrial localisation. Nevertheless, our finding on the predicted structure showed a conserved active domain of TgEndoG, suggesting that it might show pro-apoptotic nuclease activity, possibly being responsible for the occurrence of DNA fragmentation as observed in T. gondii following treatment with pro-apoptotic stimuli.

In order to determine regulation of apoptosis-like cell death in $T$. gondii, MitoTracker staining was used to examine the mitochondrial membrane potential $(\Delta \Psi \mathrm{m})$ upon cell death induction. The majority of parasites retained an intact $\Delta \Psi \mathrm{m}$ following staurosporine treatment. Conversely, miltefosine treatment led to a significant loss of $\Delta \Psi \mathrm{m}$ in the majority of parasites. Although protozoa do not express genuine caspases, caspaselike activities have been identified during cell death in other unicellular organisms. Using a CaspaTag assay, a method for in situ labelling of active caspase, we observed a rapid and strong caspase-like activity in parasites after miltefosine treatment. In contrast, after staurosporine treatment, caspase-like activity increased only transiently and to a lower extent. Importantly, in the presence of protease inhibitors (E64 and Z-VAD-FMK), the emergence of TUNEL-positive parasites was significantly abolished upon miltefosine induction, but not staurosporine.

Taken together, the results indicate the existence of an apoptosis-like cell death mechanism in T. gondii which might play a role during chronic infection. Additionally, we proof for the first time that treatment of intracellular parasites with the chemotherapeutic drug clindamycin induces an apoptosis-like cell death phenotype in a small proportion of parasites. Our results on TgBIs indicate evolutionary conserved protective effects of BI proteins from $T$. gondii to mammals and suggest a role of TgBI proteins in regulation of $T$. gondii apoptosis-like cell death. Furthermore, distinct apoptosis-like cell death pathways appear to exist in $T$. gondii; one involves both a loss of $\Delta \Psi \mathrm{m}$ and an activation of caspaselike proteases, which is specifically triggered by miltefosine. Further experiments to unravel precise mechanisms of PCD in Toxoplasma are required. A better understanding 
on Toxoplasma PCD might provide new possibilities to combat the parasite particularly during its persistent bradyzoite stage. 


\section{REFERENCES}

Al-Olayan, E.M., Williams, G.T., Hurd, H., 2002. Apoptosis in the malaria protozoan, Plasmodium berghei: a possible mechanism for limiting intensity of infection in the mosquito. International journal for parasitology 32, 1133-1143.

Ameisen, J.C., 2002. On the origin, evolution, and nature of programmed cell death: a timeline of four billion years. Cell death and differentiation 9, 367-393.

Ameisen, J.C., Idziorek, T., Billaut-Mulot, O., Loyens, M., Tissier, J.P., Potentier, A., Ouaissi, A., 1995. Apoptosis in a unicellular eukaryote (Trypanosoma cruzi): implications for the evolutionary origin and role of programmed cell death in the control of cell proliferation, differentiation and survival. Cell death and differentiation 2, 285-300.

Arnoult, D., Akarid, K., Grodet, A., Petit, P.X., Estaquier, J., Ameisen, J.C., 2002. On the evolution of programmed cell death: apoptosis of the unicellular eukaryote Leishmania major involves cysteine proteinase activation and mitochondrion permeabilization. Cell death and differentiation 9, 65-81.

Arnoult, D., Gaume, B., Karbowski, M., Sharpe, J.C., Cecconi, F., Youle, R.J., 2003. Mitochondrial release of AIF and EndoG requires caspase activation downstream of Bax/Bak-mediated permeabilization. The EMBO journal 22, 4385-4399.

Arnoult, D., Tatischeff, I., Estaquier, J., Girard, M., Sureau, F., Tissier, J.P., Grodet, A., Dellinger, M., Traincard, F., Kahn, A., Ameisen, J.C., Petit, P.X., 2001. On the evolutionary conservation of the cell death pathway: mitochondrial release of an apoptosis-inducing factor during Dictyostelium discoideum cell death. Molecular biology of the cell 12, 3016-3030.

Baggish, A.L., Hill, D.R., 2002. Antiparasitic agent atovaquone. Antimicrobial agents and chemotherapy 46, 1163-1173.

Baron, B.W., Anastasi, J., Thirman, M.J., Furukawa, Y., Fears, S., Kim, D.C., Simone, F., Birkenbach, M., Montag, A., Sadhu, A., Zeleznik-Le, N., McKeithan, T.W., 2002. The human programmed cell death-2 (PDCD2) gene is a target of BCL6 repression: implications for a role of BCL6 in the down-regulation of apoptosis. Proc Natl Acad Sci U S A 99, 2860-2865.

Baron, B.W., Hyjek, E., Gladstone, B., Thirman, M.J., Baron, J.M., 2010. PDCD2, a protein whose expression is repressed by BCL6, induces apoptosis in human cells by activation of the caspase cascade. Blood Cells Mol Dis 45, 169-175.

Belmokhtar, C.A., Hillion, J., Segal-Bendirdjian, E., 2001. Staurosporine induces apoptosis through both caspase-dependent and caspase-independent mechanisms. Oncogene 20, 3354-3362.

Bertrand, R., Solary, E., O'Connor, P., Kohn, K.W., Pommier, Y., 1994. Induction of a common pathway of apoptosis by staurosporine. Experimental cell research 211, 314-321.

Blader, I.J., Saeij, J.P., 2009. Communication between Toxoplasma gondii and its host: impact on parasite growth, development, immune evasion, and virulence. APMIS : acta pathologica, microbiologica, et immunologica Scandinavica 117, 458-476. 
Bolte, S., Cordelieres, F.P., 2006. A guided tour into subcellular colocalization analysis in light microscopy. Journal of microscopy 224, 213-232.

Breckenridge, D.G., Germain, M., Mathai, J.P., Nguyen, M., Shore, G.C., 2003. Regulation of apoptosis by endoplasmic reticulum pathways. Oncogene 22, 86088618.

Broker, L.E., Kruyt, F.A., Giaccone, G., 2005. Cell death independent of caspases: a review. Clinical cancer research : an official journal of the American Association for Cancer Research 11, 3155-3162.

Bruchhaus, I., Roeder, T., Rennenberg, A., Heussler, V.T., 2007. Protozoan parasites: programmed cell death as a mechanism of parasitism. Trends in parasitology 23, 376-383.

Camps, M., Arrizabalaga, G., Boothroyd, J., 2002. An rRNA mutation identifies the apicoplast as the target for clindamycin in Toxoplasma gondii. Molecular microbiology 43, 1309-1318.

Carrara, G., Saraiva, N., Gubser, C., Johnson, B.F., Smith, G.L., 2012. Six-transmembrane topology for Golgi anti-apoptotic protein (GAAP) and Bax inhibitor 1 (BI-1) provides model for the transmembrane Bax inhibitor-containing motif (TMBIM) family. The Journal of biological chemistry 287, 15896-15905.

Ch'ng, J.H., Kotturi, S.R., Chong, A.G., Lear, M.J., Tan, K.S., 2010. A programmed cell death pathway in the malaria parasite Plasmodium falciparum has general features of mammalian apoptosis but is mediated by clan CA cysteine proteases. Cell death $\&$ disease 1, e26.

Chae, H.J., Kim, H.R., Xu, C., Bailly-Maitre, B., Krajewska, M., Krajewski, S., Banares, S., Cui, J., Digicaylioglu, M., Ke, N., Kitada, S., Monosov, E., Thomas, M., Kress, C.L., Babendure, J.R., Tsien, R.Y., Lipton, S.A., Reed, J.C., 2004. BI-1 regulates an apoptosis pathway linked to endoplasmic reticulum stress. Molecular cell 15, 355-366.

Debache, K., Hemphill, A., 2012. Effects of miltefosine treatment in fibroblast cell cultures and in mice experimentally infected with Neospora caninum tachyzoites. Parasitology 139, 934-944.

Debrabant, A., Nakhasi, H., 2003. Programmed cell death in trypanosomatids: is it an altruistic mechanism for survival of the fittest? Kinetoplastid biology and disease 2, 7.

Dorlo, T.P., Balasegaram, M., Beijnen, J.H., de Vries, P.J., 2012. Miltefosine: a review of its pharmacology and therapeutic efficacy in the treatment of leishmaniasis. $J$ Antimicrob Chemother.

Dubey, J.P., 1995. Duration of immunity to shedding of Toxoplasma gondii oocysts by cats. The Journal of parasitology 81, 410-415.

Dubey, J.P., 1998. Advances in the life cycle of Toxoplasma gondii. International journal for parasitology 28, 1019-1024.

Dubey, J.P., 2008. The history of Toxoplasma gondii--the first 100 years. The Journal of eukaryotic microbiology 55, 467-475.

Dubey, J.P., Lindsay, D.S., Speer, C.A., 1998. Structures of Toxoplasma gondii tachyzoites, bradyzoites, and sporozoites and biology and development of tissue cysts. Clinical microbiology reviews 11, 267-299. 
Duszenko, M., Figarella, K., Macleod, E.T., Welburn, S.C., 2006. Death of a trypanosome: a selfish altruism. Trends in parasitology 22, 536-542.

Dzierszinski, F., Nishi, M., Ouko, L., Roos, D.S., 2004. Dynamics of Toxoplasma gondii differentiation. Eukaryotic cell 3, 992-1003.

El-Fadili, A.K., Zangger, H., Desponds, C., Gonzalez, I.J., Zalila, H., Schaff, C., Ives, A., Masina, S., Mottram, J.C., Fasel, N., 2010. Cathepsin B-like and cell death in the unicellular human pathogen Leishmania. Cell death \& disease 1, e71.

Elmore, S., 2007. Apoptosis: a review of programmed cell death. Toxicologic pathology 35, 495-516.

Fichera, M.E., Bhopale, M.K., Roos, D.S., 1995. In vitro assays elucidate peculiar kinetics of clindamycin action against Toxoplasma gondii. Antimicrobial agents and chemotherapy 39, 1530-1537.

Figarella, K., Rawer, M., Uzcategui, N.L., Kubata, B.K., Lauber, K., Madeo, F., Wesselborg, S., Duszenko, M., 2005. Prostaglandin D2 induces programmed cell death in Trypanosoma brucei bloodstream form. Cell death and differentiation 12, 335-346.

Filisetti, D., Candolfi, E., 2004. Immune response to Toxoplasma gondii. Annali dell'Istituto superiore di sanita 40, 71-80.

Frenkel, J.K., Dubey, J.P., Miller, N.L., 1970. Toxoplasma gondii in cats: fecal stages identified as coccidian oocysts. Science 167, 893-896.

Gajria, B., Bahl, A., Brestelli, J., Dommer, J., Fischer, S., Gao, X., Heiges, M., Iodice, J., Kissinger, J.C., Mackey, A.J., Pinney, D.F., Roos, D.S., Stoeckert, C.J., Jr., Wang, H., Brunk, B.P., 2008. ToxoDB: an integrated Toxoplasma gondii database resource. Nucleic acids research 36, D553-556.

Galluzzi, L., Vitale, I., Abrams, J.M., Alnemri, E.S., Baehrecke, E.H., Blagosklonny, M.V., Dawson, T.M., Dawson, V.L., El-Deiry, W.S., Fulda, S., Gottlieb, E., Green, D.R., Hengartner, M.O., Kepp, O., Knight, R.A., Kumar, S., Lipton, S.A., Lu, X., Madeo, F., Malorni, W., Mehlen, P., Nunez, G., Peter, M.E., Piacentini, M., Rubinsztein, D.C., Shi, Y., Simon, H.U., Vandenabeele, P., White, E., Yuan, J., Zhivotovsky, B., Melino, G., Kroemer, G., 2012. Molecular definitions of cell death subroutines: recommendations of the Nomenclature Committee on Cell Death 2012. Cell death and differentiation 19, 107-120.

Gannavaram, S., Vedvyas, C., Debrabant, A., 2008. Conservation of the pro-apoptotic nuclease activity of endonuclease $\mathrm{G}$ in unicellular trypanosomatid parasites. Journal of cell science 121, 99-109.

Green, D.R., 2011. Means to an End: Apoptosis and Other Cell Death Mechanisms. Cold Spring Harbor Laboratory Press, New York.

Gumienny, T.L., Brugnera, E., Tosello-Trampont, A.C., Kinchen, J.M., Haney, L.B., Nishiwaki, K., Walk, S.F., Nemergut, M.E., Macara, I.G., Francis, R., Schedl, T., Qin, Y., Van Aelst, L., Hengartner, M.O., Ravichandran, K.S., 2001. CED12/ELMO, a novel member of the CrkII/Dock180/Rac pathway, is required for phagocytosis and cell migration. Cell 107, 27-41.

Hetz, C.A., 2007. ER stress signaling and the BCL-2 family of proteins: from adaptation to irreversible cellular damage. Antioxidants \& redox signaling 9, 2345-2355. 
Hill, D., Dubey, J.P., 2002. Toxoplasma gondii: transmission, diagnosis and prevention. Clinical microbiology and infection : the official publication of the European Society of Clinical Microbiology and Infectious Diseases 8, 634-640.

Hu, K., Roos, D.S., Angel, S.O., Murray, J.M., 2004. Variability and heritability of cell division pathways in Toxoplasma gondii. Journal of cell science 117, 5697-5705.

Huckelhoven, R., 2004. BAX Inhibitor-1, an ancient cell death suppressor in animals and plants with prokaryotic relatives. Apoptosis : an international journal on programmed cell death 9, 299-307.

Hurd, H., Grant, K.M., Arambage, S.C., 2006. Apoptosis-like death as a feature of malaria infection in mosquitoes. Parasitology 132 Suppl, S33-47.

Innes, E.A., 2010. A brief history and overview of Toxoplasma gondii. Zoonoses and public health 57, 1-7.

Jaattela, M., Tschopp, J., 2003. Caspase-independent cell death in T lymphocytes. Nature immunology 4, 416-423.

James, E.R., Green, D.R., 2004. Manipulation of apoptosis in the host-parasite interaction. Trends in parasitology 20, 280-287.

Jeong, S.Y., Seol, D.W., 2008. The role of mitochondria in apoptosis. BMB reports 41, 1122.

Jiménez-Ruiz, A., Alzate, J.F., MacLeod, E.T., Lüder, C.G.K., Fasel, N., Hurd, H., 2010. Apoptotic markers in protozoan parasites. Parasites \& Vectors 3.

Jones, B.E., Lo, C.R., Srinivasan, A., Valentino, K.L., Czaja, M.J., 1999. Ceramide induces caspase-independent apoptosis in rat hepatocytes sensitized by inhibition of RNA synthesis. Hepatology 30, 215-222.

Kerr, J.F., Wyllie, A.H., Currie, A.R., 1972. Apoptosis: a basic biological phenomenon with wide-ranging implications in tissue kinetics. British journal of cancer 26, 239257.

Kim, H.R., Lee, G.H., Ha, K.C., Ahn, T., Moon, J.Y., Lee, B.J., Cho, S.G., Kim, S., Seo, Y.R., Shin, Y.J., Chae, S.W., Reed, J.C., Chae, H.J., 2008. Bax Inhibitor-1 Is a pHdependent regulator of $\mathrm{Ca} 2+$ channel activity in the endoplasmic reticulum. The Journal of biological chemistry 283, 15946-15955.

Kim, K., Weiss, L.M., 2004. Toxoplasma gondii: the model apicomplexan. International journal for parasitology 34, 423-432.

Kroemer, G., Galluzzi, L., Brenner, C., 2007. Mitochondrial membrane permeabilization in cell death. Physiol Rev 87, 99-163.

Kroemer, G., Galluzzi, L., Vandenabeele, P., Abrams, J., Alnemri, E.S., Baehrecke, E.H., Blagosklonny, M.V., El-Deiry, W.S., Golstein, P., Green, D.R., Hengartner, M., Knight, R.A., Kumar, S., Lipton, S.A., Malorni, W., Nunez, G., Peter, M.E., Tschopp, J., Yuan, J., Piacentini, M., Zhivotovsky, B., Melino, G., Nomenclature Committee on Cell, D., 2009. Classification of cell death: recommendations of the Nomenclature Committee on Cell Death 2009. Cell death and differentiation 16, 311.

Krysko, D.V., Vanden Berghe, T., D'Herde, K., Vandenabeele, P., 2008. Apoptosis and necrosis: detection, discrimination and phagocytosis. Methods 44, 205-221.

Laliberte, J., Carruthers, V.B., 2008. Host cell manipulation by the human pathogen Toxoplasma gondii. Cellular and molecular life sciences : CMLS 65, 1900-1915. 
Lee, S.H., Meng, X.W., Flatten, K.S., Loegering, D.A., Kaufmann, S.H., 2013. Phosphatidylserine exposure during apoptosis reflects bidirectional trafficking between plasma membrane and cytoplasm. Cell death and differentiation 20, 64-76.

Lippincott-Schwartz, J., Yuan, L.C., Bonifacino, J.S., Klausner, R.D., 1989. Rapid redistribution of Golgi proteins into the ER in cells treated with brefeldin A: evidence for membrane cycling from Golgi to ER. Cell 56, 801-813.

Lirussi, D., Matrajt, M., 2011. RNA granules present only in extracellular toxoplasma gondii increase parasite viability. International journal of biological sciences 7 , 960-967.

Lisbona, F., Rojas-Rivera, D., Thielen, P., Zamorano, S., Todd, D., Martinon, F., Glavic, A., Kress, C., Lin, J.H., Walter, P., Reed, J.C., Glimcher, L.H., Hetz, C., 2009. BAX inhibitor-1 is a negative regulator of the ER stress sensor IRE1alpha. Molecular cell 33, 679-691.

Liu, L.F., Desai, S.D., Li, T.K., Mao, Y., Sun, M., Sim, S.P., 2000. Mechanism of action of camptothecin. Annals of the New York Academy of Sciences 922, 1-10.

Low, R.L., 2003. Mitochondrial Endonuclease $G$ function in apoptosis and mtDNA metabolism: a historical perspective. Mitochondrion 2, 225-236.

Luder, C.G., Campos-Salinas, J., Gonzalez-Rey, E., van Zandbergen, G., 2010. Impact of protozoan cell death on parasite-host interactions and pathogenesis. Parasit Vectors $3,116$.

Madeo, F., Kroemer, G., 2009. Intricate links between ER stress and apoptosis. Molecular cell 33, 669-670.

Makrides, S.C., 1996. Strategies for achieving high-level expression of genes in Escherichia coli. Microbiological reviews 60, 512-538.

Martin, S.J., Reutelingsperger, C.P., McGahon, A.J., Rader, J.A., van Schie, R.C., LaFace, D.M., Green, D.R., 1995. Early redistribution of plasma membrane phosphatidylserine is a general feature of apoptosis regardless of the initiating stimulus: inhibition by overexpression of $\mathrm{Bcl}-2$ and Abl. The Journal of experimental medicine 182, 1545-1556.

McCarthy, J.V., Cotter, T.G., 1997. Cell shrinkage and apoptosis: a role for potassium and sodium ion efflux. Cell death and differentiation 4, 756-770.

McFadden, D.C., Tomavo, S., Berry, E.A., Boothroyd, J.C., 2000. Characterization of cytochrome $\mathrm{b}$ from Toxoplasma gondii and $\mathrm{Q}(\mathrm{o})$ domain mutations as a mechanism of atovaquone-resistance. Molecular and biochemical parasitology 108, 1-12.

Melo, E.J., Attias, M., De Souza, W., 2000. The single mitochondrion of tachyzoites of Toxoplasma gondii. Journal of structural biology 130, 27-33.

Miller, S.G., Carnell, L., Moore, H.H., 1992. Post-Golgi membrane traffic: brefeldin A inhibits export from distal Golgi compartments to the cell surface but not recycling. The Journal of cell biology 118, 267-283.

Montoya, J.G., Remington, J.S., 2008. Management of Toxoplasma gondii infection during pregnancy. Clinical infectious diseases : an official publication of the Infectious Diseases Society of America 47, 554-566.

Nagata, S., Nagase, H., Kawane, K., Mukae, N., Fukuyama, H., 2003. Degradation of chromosomal DNA during apoptosis. Cell death and differentiation 10, 108-116. 
Nedelcu, A.M., 2009. Comparative genomics of phylogenetically diverse unicellular eukaryotes provide new insights into the genetic basis for the evolution of the programmed cell death machinery. Journal of molecular evolution 68, 256-268.

Nishi, M., Hu, K., Murray, J.M., Roos, D.S., 2008. Organellar dynamics during the cell cycle of Toxoplasma gondii. Journal of cell science 121, 1559-1568.

Paris, C., Loiseau, P.M., Bories, C., Breard, J., 2004. Miltefosine induces apoptosis-like death in Leishmania donovani promastigotes. Antimicrobial agents and chemotherapy 48, 852-859.

Parussini, F., Coppens, I., Shah, P.P., Diamond, S.L., Carruthers, V.B., 2010. Cathepsin L occupies a vacuolar compartment and is a protein maturase within the endo/exocytic system of Toxoplasma gondii. Molecular microbiology 76, 13401357.

Pavesio, C.E., Chiappino, M.L., Setzer, P.Y., Nichols, B.A., 1992. Toxoplasma gondii: differentiation and death of bradyzoites. Parasitology research 78, 1-9.

Peng, B.W., Lin, J., Lin, J.Y., Jiang, M.S., Zhang, T., 2003. Exogenous nitric oxide induces apoptosis in Toxoplasma gondii tachyzoites via a calcium signal transduction pathway. Parasitology 126, 541-550.

Pfefferkorn, E.R., Nothnagel, R.F., Borotz, S.E., 1992. Parasiticidal effect of clindamycin on Toxoplasma gondii grown in cultured cells and selection of a drug-resistant mutant. Antimicrobial agents and chemotherapy 36, 1091-1096.

Popov, L.S., Korochkin, L.I., 2004. [Genetically programmed cell death (apoptosis)]. Genetika 40, 149-166.

Proto, W.R., Coombs, G.H., Mottram, J.C., 2013. Cell death in parasitic protozoa: regulated or incidental? Nature reviews. Microbiology 11, 58-66.

Que, X., Engel, J.C., Ferguson, D., Wunderlich, A., Tomavo, S., Reed, S.L., 2007. Cathepsin Cs are key for the intracellular survival of the protozoan parasite, Toxoplasma gondii. The Journal of biological chemistry 282, 4994-5003.

Que, X., Ngo, H., Lawton, J., Gray, M., Liu, Q., Engel, J., Brinen, L., Ghosh, P., Joiner, K.A., Reed, S.L., 2002. The cathepsin B of Toxoplasma gondii, toxopain-1, is critical for parasite invasion and rhoptry protein processing. The Journal of biological chemistry 277, 25791-25797.

Reece, S.E., Pollitt, L.C., Colegrave, N., Gardner, A., 2011. The meaning of death: evolution and ecology of apoptosis in protozoan parasites. PLoS pathogens 7, e1002320.

Rico, E., Alzate, J.F., Arias, A.A., Moreno, D., Clos, J., Gago, F., Moreno, I., Dominguez, M., Jimenez-Ruiz, A., 2009. Leishmania infantum expresses a mitochondrial nuclease homologous to EndoG that migrates to the nucleus in response to an apoptotic stimulus. Molecular and biochemical parasitology 163, 28-38.

Robinson, K.S., Clements, A., Williams, A.C., Berger, C.N., Frankel, G., 2011. Bax inhibitor 1 in apoptosis and disease. Oncogene 30, 2391-2400.

Rosano, G.L., Ceccarelli, E.A., 2009. Rare codon content affects the solubility of recombinant proteins in a codon bias-adjusted Escherichia coli strain. Microbial cell factories 8,41 .

Rozman-Pungercar, J., Kopitar-Jerala, N., Bogyo, M., Turk, D., Vasiljeva, O., Stefe, I., Vandenabeele, P., Bromme, D., Puizdar, V., Fonovic, M., Trstenjak-Prebanda, M., Dolenc, I., Turk, V., Turk, B., 2003. Inhibition of papain-like cysteine proteases 
and legumain by caspase-specific inhibitors: when reaction mechanism is more important than specificity. Cell Death Differ 10, 881-888.

Saeij, J.P., Boyle, J.P., Boothroyd, J.C., 2005. Differences among the three major strains of Toxoplasma gondii and their specific interactions with the infected host. Trends in parasitology 21, 476-481.

Santos, T.A., Portes Jde, A., Damasceno-Sa, J.C., Caldas, L.A., Souza, W., Damatta, R.A., Seabra, S.H., 2011. Phosphatidylserine exposure by Toxoplasma gondii is fundamental to balance the immune response granting survival of the parasite and of the host. PloS one 6, e27867.

Schafer, P., Scholz, S.R., Gimadutdinow, O., Cymerman, I.A., Bujnicki, J.M., RuizCarrillo, A., Pingoud, A., Meiss, G., 2004. Structural and functional characterization of mitochondrial EndoG, a sugar non-specific nuclease which plays an important role during apoptosis. Journal of molecular biology 338, 217 228.

Scorrano, L., Oakes, S.A., Opferman, J.T., Cheng, E.H., Sorcinelli, M.D., Pozzan, T., Korsmeyer, S.J., 2003. BAX and BAK regulation of endoplasmic reticulum $\mathrm{Ca} 2+$ : a control point for apoptosis. Science 300, 135-139.

Seabra, S.H., de Souza, W., Damatta, R.A., 2004. Toxoplasma gondii exposes phosphatidylserine inducing a TGF-betal autocrine effect orchestrating macrophage evasion. Biochemical and biophysical research communications 324, 744-752.

Shi, Y., 2002. Mechanisms of caspase activation and inhibition during apoptosis. Molecular cell 9, 459-470.

Sibley, L.D., Ajioka, J.W., 2008. Population structure of Toxoplasma gondii: clonal expansion driven by infrequent recombination and selective sweeps. Annual review of microbiology 62, 329-351.

Sibley, L.D., Khan, A., Ajioka, J.W., Rosenthal, B.M., 2009. Genetic diversity of Toxoplasma gondii in animals and humans. Philosophical transactions of the Royal Society of London. Series B, Biological sciences 364, 2749-2761.

Su, C., Evans, D., Cole, R.H., Kissinger, J.C., Ajioka, J.W., Sibley, L.D., 2003. Recent expansion of Toxoplasma through enhanced oral transmission. Science 299, 414416.

Tait, S.W., Green, D.R., 2008. Caspase-independent cell death: leaving the set without the final cut. Oncogene 27, 6452-6461.

Taylor, R.C., Cullen, S.P., Martin, S.J., 2008. Apoptosis: controlled demolition at the cellular level. Nature reviews. Molecular cell biology 9, 231-241.

Tenter, A.M., Heckeroth, A.R., Weiss, L.M., 2000. Toxoplasma gondii: from animals to humans. International journal for parasitology 30, 1217-1258.

Troyano, A., Sancho, P., Fernandez, C., de Blas, E., Bernardi, P., Aller, P., 2003. The selection between apoptosis and necrosis is differentially regulated in hydrogen peroxide-treated and glutathione-depleted human promonocytic cells. Cell death and differentiation 10, 889-898.

van Loo, G., Schotte, P., van Gurp, M., Demol, H., Hoorelbeke, B., Gevaert, K., Rodriguez, I., Ruiz-Carrillo, A., Vandekerckhove, J., Declercq, W., Beyaert, R., Vandenabeele, P., 2001. Endonuclease G: a mitochondrial protein released in 
apoptosis and involved in caspase-independent DNA degradation. Cell death and differentiation 8, 1136-1142.

van Zandbergen, G., Bollinger, A., Wenzel, A., Kamhawi, S., Voll, R., Klinger, M., Muller, A., Holscher, C., Herrmann, M., Sacks, D., Solbach, W., Laskay, T., 2006. Leishmania disease development depends on the presence of apoptotic promastigotes in the virulent inoculum. Proceedings of the National Academy of Sciences of the United States of America 103, 13837-13842.

van Zandbergen, G., Lüder, C.G.K., Heussler, V., Duszenko, M., 2010. Programmed cell death in unicellular parasites: a prerequisite for sustained infection? Trends in parasitology 26, 477-483.

Vandenabeele, P., Orrenius, S., Zhivotovsky, B., 2005. Serine proteases and calpains fulfill important supporting roles in the apoptotic tragedy of the cellular opera. Cell death and differentiation 12, 1219-1224.

Vaux, D.L., Strasser, A., 1996. The molecular biology of apoptosis. Proceedings of the National Academy of Sciences of the United States of America 93, 2239-2244.

Vermes, I., Haanen, C., Steffens-Nakken, H., Reutelingsperger, C., 1995. A novel assay for apoptosis. Flow cytometric detection of phosphatidylserine expression on early apoptotic cells using fluorescein labelled Annexin V. Journal of immunological methods 184, 39-51.

Vitale, I., Galluzzi, L., Castedo, M., Kroemer, G., 2011. Mitotic catastrophe: a mechanism for avoiding genomic instability. Nature reviews. Molecular cell biology 12, 385392.

Wang, C., Youle, R.J., 2009. The role of mitochondria in apoptosis*. Annual review of genetics 43, 95-118.

Watanabe, N., Lam, E., 2009. Bax inhibitor-1, a conserved cell death suppressor, is a key molecular switch downstream from a variety of biotic and abiotic stress signals in plants. International journal of molecular sciences 10, 3149-3167.

Weiss, L.M., Kim, K., 2000. The development and biology of bradyzoites of Toxoplasma gondii. Frontiers in bioscience : a journal and virtual library 5, D391-405.

Xu, C., Bailly-Maitre, B., Reed, J.C., 2005. Endoplasmic reticulum stress: cell life and death decisions. The Journal of clinical investigation 115, 2656-2664.

Xu, Q., Reed, J.C., 1998. Bax inhibitor-1, a mammalian apoptosis suppressor identified by functional screening in yeast. Molecular cell 1, 337-346.

Zhang, X.D., Gillespie, S.K., Hersey, P., 2004. Staurosporine induces apoptosis of melanoma by both caspase-dependent and -independent apoptotic pathways. Molecular cancer therapeutics 3, 187-197.

Zimmermann, K.C., Bonzon, C., Green, D.R., 2001. The machinery of programmed cell death. Pharmacology \& therapeutics 92, 57-70.

Zimmermann, K.C., Ricci, J.E., Droin, N.M., Green, D.R., 2002. The role of ARK in stress-induced apoptosis in Drosophila cells. J Cell Biol 156, 1077-1087. 


\section{PUBLICATIONS}

\section{ORIGINAL PUBLICATIONS}

Some parts of this study have been published as an original paper in Apoptosis journal.

Ni Nyoman, A.D., Lüder, C.G.K., 2013. Apoptosis-like cell death pathways in the unicellular parasite Toxoplasma gondii following treatment with apoptosis inducers and chemotherapeutic agents: a proof-of-concept study. Apoptosis 18, 664-680.

DOI 10.1007/s10495-013-0832-8.

\section{ABSTRACTS/PRESENTATIONS}

1. Ni Nyoman, AD., Klages, N., Soldati-Favre, D., Lüder, CGK. Heterologous Expression of Putative Toxoplasma gondii Bax Inhibitors Suggest a Role in Programmed Cell Death (PCD) Regulation. $3^{\text {rd }}$ COST Action BM0802 (Live or Death of Protozoan Parasites/LODOPP), Brussels, 27-29 March, 2011 (oral presentation).

2. Ni Nyoman, AD., Klages, N., Soldati-Favre, D., Lüder, CGK. Identification of Apoptosis-Like Markers and Regulators in Toxoplasma gondii Programmed Cell Death (PCD). $63^{\text {rd }}$ Annual Meeting German Society for Hygiene and Microbiology (DGHM), Essen, 25-28 September, 2011 (oral presentation).

3. Ni Nyoman, AD., Klages, N., Soldati-Favre, D., Lüder, CGK. Characterization of Apoptosis-Like Cell Death and Putative Cell Death Regulators in Toxoplasma gondii. $4^{\text {th }}$ COST Action BM0802 (Live or Death of Protozoan Parasites/LODOPP), Milan, 19-21 January, 2012 (oral presentation).

4. Ni Nyoman, AD., Klages, N., Soldati-Favre, D., Lüder, CGK. Apoptosis-Like Markers and Putative Cell Death Regulators in Toxoplasma gondii. 25 ${ }^{\text {th }}$ Annual Meeting German Society for Parasitology, Heidelberg, 14-17 March, 2012 (oral presentation).

5. Ni Nyoman, AD. Programmed Cell Death in Toxoplasma gondii- a research project. DAAD Summer School "Indonesian-German Health Education Partnership", Göttingen, 24 June-3 July 2012 (oral presentation). 
6. Ni Nyoman, AD., Lüder, CGK. Apoptosis-like Cell Death in The Protozoan Toxoplasma gondii: When Does It Matter? $64^{\text {th }}$ Annual Meeting German Society for Hygiene and Microbiology (DGHM), Hamburg, 30 September-3 October, 2012 (poster presentation).

7. Ni Nyoman, AD., Lüder, CGK. Apoptosis-like Cell Death in Toxoplasma gondii: Purpose of an End. $5^{\text {th }}$ COST Action BM0802 (Live or Death of Protozoan Parasites/LODOPP), Edinburgh, 9-10 October, 2012. The talk was presented by Carsten Lüder (oral presentation).

8. Ayu Dewi Ni Nyoman, Carsten GK Lüder. Pro-apoptotic Stimuli Trigger Distinctive Programmed Cell Death Pathways in Toxoplasma gondii. Workshop of the DGHM working group on eukaryotic pathogens, Hannover, 01-02 February, 2013 (oral presentation). 


\section{CURRICULUM VITAE}

\section{CONTACT INFORMATION}

Name

: Ni Nyoman Ayu Dewi

Present address

: Christophorusweg 12, 37075 Göttingen, Germany

Home address

: J1. Kemuda II/14 Tonja, Denpasar-Bali 80115, Indonesia

Email

:saptadie@yahoo.com

\section{PERSONAL INFORMATION}

Date of Birth

: 10 November 1977

Place of Birth

: Denpasar

Citizenship

: Indonesia

Sex

: Female

Marital Status

: Married

\section{EDUCATION}

1995-2001

: Bachelor program, Faculty of Medicine, Udayana University, Denpasar-Bali, Indonesia

2003-2006

: Master program, Center for Biotechnology, Gadjah Mada

University, Yogyakarta, Indonesia

(DUE-like scholarship-Indonesia)

Thesis: "Cloning Gene Encoding Microneme 3 of Tachyzoites Toxoplasma gondii Local Isolate"

2009-2013

: Doctoral program, Institute for Medical Microbiology,

Georg-August University, Göttingen, Germany

(DAAD scholarship-Germany) 


\section{EMPLOYMENT AND AFFILIATIONS}

2002-present

: Academic and research staff in Department of Biochemistry Faculty of Medicine and Health Sciences, Udayana University, Denpasar-Bali, Indonesia

2002-present : Member of Indonesian Medical Association

Member of Indonesian Molecular Biology and Biochemistry Association

LANGUAGE : Indonesian (native), German (intermediate- B1 level), English (good)

INTERESTS : Travelling and Reading 